\title{
Scaling black holes and modularity
}

\section{Aradhita Chattopadhyaya, Jan Manschot and Swapnamay Mondal}

School of Mathematics, Trinity College, Dublin 2, Ireland

Hamilton Mathematical Institute, Trinity College, Dublin 2, Ireland

E-mail: aradhita@maths.tcd.ie, manschot@maths.tcd.ie, swapno@maths.tcd.ie

ABSTRACT: Scaling black holes are solutions of supergravity with multiple black hole singularities, which can be adiabatically connected to a single center black hole solution. We develop techniques to determine partition functions for such scaling black holes, if each constituent carries a non-vanishing magnetic charge corresponding to a D4-brane in string theory, or equivalently M5-brane in M-theory. For three constituents, we demonstrate that the partition function is a mock modular form of depth two, and we determine the appropriate non-holomorphic completion using generalized error functions. From the fourdimensional perspective, the modular parameter is the axion-dilaton, and our results show that $S$-duality leaves this subset of the spectrum invariant. From the five-dimensional perspective, the modular parameter is the complex structure of a torus $T^{2}$, and the scaling black holes are dual to states in the dimensional reduction of the M5-brane worldvolume theory to $T^{2}$. As a case study, we specialize the compactification manifold to a K3 fibration, and explicitly evaluate holomorphic parts of partition functions.

KeYwords: Black Holes in String Theory, AdS-CFT Correspondence, D-Branes, M-Theory

ArXIV EPRINT: 2110.05504 


\section{Contents}

1 Introduction 1

2 Black hole solutions in $\mathcal{N}=2$ supergravity 5

2.1 Black hole bound states 5

2.2 Bound state indices 8

3 Review of D4-brane black holes 13

3.1 Supergravity partition function 13

$\begin{array}{lll}3.2 & \text { Attractor partition function } & 16\end{array}$

4 Partition functions for scaling black holes $\quad 17$

$\begin{array}{lll}4.1 & \text { Bound states and lattices } & 19\end{array}$

4.2 Partition functions 26

4.3 Convergence 28

4.4 Modular completion of $\Phi_{\mu} \quad 29$

$\begin{array}{lll}4.5 & \text { Modular completion of } \Psi_{\mu} & 35\end{array}$

5 Relation to M5-branes and $\mathrm{AdS}_{3} / \mathrm{CFT}_{2} \quad 37$

6 Case studies $\quad 39$

6.1 Charge configuration 1: $P_{1}=P_{2}=(0,1), P_{3}=(1,1) \quad 40$

6.2 Charge configuration 2: $P_{1}=(1,0), P_{2}=P_{3}=(0,1)$

\section{Introduction}

Solutions of supergravity with multiple black hole singularities provide interesting insights on the spectrum of quantum gravity and the dependence on the compactification moduli [1-10]. Such multi-center solutions are particularly intriguing if their solution space contains a region, where the distances between the centers becomes arbitrarily small. In other words, these solutions, known as "scaling" solutions $[6,11]$ in four dimensions, are adiabatically connected to a supergravity solution with a single black hole singularity.

We will study such scaling black holes formed from M5-branes in M-theory, or equivalently D4-branes in Type IIA string theory [12]. Such black holes are well studied using the $\mathrm{AdS}_{3} / \mathrm{CFT}_{2}$ correspondence [12-15], as well as the hypermultiplet geometry of the IIB string theory [16-18]. The D4/M5-branes wrap a four-cycle (or divisor) $P \in H_{4}(X, \mathbb{Z})$ of the Calabi-Yau threefold $X$, which can also be studied within algebraic geometry [19-23].

Multi-center solutions exist with each center having a positive D4-brane charge but vanishing D6-brane charge. Their walls of marginal stability extend to the large volume 
regime of the Kähler moduli space [24-27], which corresponds to $\operatorname{Im}(t)=J=\lambda \underline{J}$, where $\underline{J}$ is the normalized, dimensionless Kähler modulus, $\underline{J}^{3}=1$, and $\lambda \gg 1$. While attractor points lie typically in the interior of the Kähler moduli space, there is an analogue of the attractor point at large volume $t_{\gamma}^{\lambda}$ defined in eq. (3.14) $[18,25,27]$. Similarly, single center black holes, whose internal degrees of freedom are independent of the asymptotic moduli, have an analogue at large volume, where families of black hole solutions appear effectively as single center black holes. Much like nucleons can be considered as elementary particles at sufficiently low energies.

To deal with these effectively single center solutions, we introduce the notion of " $\lambda$-core", or "core" for short. The defining property of a " $\lambda$-core" is that the distances between constituents in a $\lambda$-core are bounded by $C \lambda^{-3}$ for sufficiently large $\lambda$, and for some fixed length $C$. Examples of such bound black holes states are of course proper single center black holes, as well as bound states of a D6-brane and anti-D6-brane, for which the wall of marginal stability lies in the interior of the Kähler moduli space.

The $\lambda$-core solutions are also distinguished in the uplift to five dimensions, and decoupling limit. Recall that the four dimensional Newton's constant equals $G_{4}=\ell_{5}^{3} / R$, with $\ell_{5}$ the five dimensional Planck length and $R$ the radius of the M-theory circle. Then, $\lambda$ is related to these variables by $\lambda=R / \ell_{5}$ [27]. When uplifted to five dimensions and in the decoupling limit $\ell_{5} \rightarrow 0$, states with D4-D2-D0 charges and within a $\lambda$-core will develop an asymptotically $\mathrm{AdS}_{3}$ throat, whereas bound states with larger separation will decouple from the spectrum since the energy of their fluctuations diverges [27].

In a series of works $[18,28-30]$, the modular properties of partition functions enumerating D4-D2-D0 black holes are studied from a complementary perspective, namely by mapping this D-brane system to D3-D1-D(-1) instantons. These D-instantons correct the hypermultiplet geometry [17,31-33], which is constrained by IIB $\mathrm{SL}(2, \mathbb{Z})$ S-duality group. This duality group acts on the axion-dilation field $\chi:=C^{(0)}+i e^{-\phi}$ by linear fractional transformations. Here $C^{(0)}$ is the $\mathrm{RR}$ scalar and $\phi$ is the dilaton. Alternatively, the duality can be identified with the modular symmetry of the worldvolume reduction of M5-branes. These have proven to be fruitful connections to determine the modular and analytic properties of the partition functions for D4-D2-D0 black holes. In this way, non-holomorphic contributions to the partition function are determined, which imply that the partition functions involve mock modular forms [34, 35], and mock modular forms of higher depth [36-39]. This implies potentially interesting arithmetic of the BPS indices, while the non-holomorphic contribution is also interesting independently, and a generalization of similar non-holomorphic terms in partition functions of $\mathcal{N}=4$ Yang-Mills on four-manifolds [40, 41]. The origins and explicit expressions of these terms have been understood better recently [30, 42-48].

We will study in this paper the partition function of scaling black holes with three constituents. Each constituent is a $\lambda$-core carrying a positive D4-brane charge, but vanishing D6-brane charge. Intriguingly, each core gives rise to an associated $\mathrm{AdS}_{3} / \mathrm{CFT}_{2}$ after uplifting to M-theory, while the near coincident region gives rise to an asymptotic $\mathrm{AdS}_{3}$ solution for the total magnetic charge, and should thus be captured by the $\mathrm{AdS}_{3} / \mathrm{CFT}_{2}$ correspondence for the total charge. In other words, they are examples of AdS fragmentation, 
and attributed to the Coulomb branch of the CFT [1]. The existence of scaling black holes with these charges puts constraints on the topology of the Calabi-Yau threefold. In particular, these only exist if the second Betti number of the Calabi-Yau manifold $b_{2} \geq 2$.

Our explicit analysis of scaling solutions gives rise to a decomposition for the partition function of attractor black holes $\mathcal{Z}_{P}^{\lambda}$ with fixed magnetic charge $P$ in terms of partition functions of single core and $n$-core scaling black holes $\mathcal{Z}_{P}^{n T}$. This reads explicitly (See also eq. (4.63).),

$$
\mathcal{Z}_{P}^{\lambda}=\mathcal{Z}_{P}^{T}+\mathcal{Z}_{P}^{3 T}+\ldots
$$

where $\mathcal{Z}_{P}^{T}$ is the partition function of single core black holes, $\mathcal{Z}_{P}^{3 T}$ the partition function of scaling black holes consistenting of three cores, and the dots stand for scaling black holes with $n>3$ cores. We determine the holomorphic part of the partition function using formula's for the degeneracies of black hole bound states such as studied in [6, 11, 49-53], which gives rise to a holomorphic indefinite theta series $\Psi_{\mu}$ of signature $\left(2,2 b_{2}-2\right)$. Since its coefficients grow polynomially, this demonstrates that the entropy arising from these solutions is exponentially smaller than the entropy of a single center black hole. This is expected since we have not included pure Higgs degeneracies [11, 49] or single center [51]. We have worked out two explicit case studies, where we specialize the CY three-fold to a K3 fibration and determine explicit $q$-series for the partition function. We do observe that the exponent of the leading term in the $q$-expansions is rather large.

We have moreover demonstrated that the partition function $\mathcal{Z}_{P}^{3 T}$ admits a theta function decomposition as a consequence of a spectral flow symmetry familiar from MSW CFT. This in contrast to generic bound states with non-vanishing D4-brane charge for which this symmetry is not present [25]. On the other hand for modular transformations, $\mathcal{Z}_{P}^{3 T}$ needs to be complemented with additional non-holomorphic terms, a phenomena which is also familiar for the attractor partition function $\mathcal{Z}_{P}^{\lambda}$ as mentioned above. We distinguish the completed functions from the original functions by a hat, thus $\widehat{\mathcal{Z}}_{P}^{\lambda}$ for $\mathcal{Z}_{P}^{\lambda}$ and $\widehat{\mathcal{Z}}_{P}^{3 T}$ for $\mathcal{Z}_{P}^{3 T}$ and similarly for other functions. We then establish that $\widehat{\mathcal{Z}}_{P}^{\lambda}$ and $\widehat{\mathcal{Z}}_{P}^{3 T}$ transform identically. Alexandrov and Pioline have derived in ref. [29] the non-holomorphic terms for the r.h.s., $\widehat{\mathcal{Z}}_{P}^{\lambda}$. It would be interesting to combine this with the non-holomorphic terms derived in the present paper for $\widehat{\mathcal{Z}}_{P}^{3 T}$ to deduce the non-holomorphic terms of $\widehat{\mathcal{Z}}_{P}^{T}$.

The identical transformation properties of $\widehat{\mathcal{Z}}_{P}^{\lambda}$ and $\widehat{\mathcal{Z}}_{P}^{n T}$ raises the question, which term(s) correspond to the partition function of the conformal field theory. Since upon taking the decoupling limit to vanishing $5 \mathrm{~d}$ Planck length $\ell_{5} \rightarrow 0$ [27], the supergravity solutions contributing to $\widehat{\mathcal{Z}}_{P}^{3 T}$ decouple from the $\mathrm{AdS}_{3}$ geometry. This term is therefore not expected to correspond to states within the MSW conformal field theory, and it seems therefore plausible to us to expect that $\widehat{\mathcal{Z}}_{P}^{T}$ is to be identified with the MSW CFT partition function. It would be interesting to understand whether the finite difference between $\widehat{\mathcal{Z}}_{P}^{T}$ and $\widehat{\mathcal{Z}}_{P}^{\lambda}$ can arise by turning on the irrelevant perturbation in the CFT, which corresponds to moving away from the near horizon geometry and up the attractor flow [54].

Determination of the completion $\widehat{\mathcal{Z}}_{P}^{3 T}$ amounts to determining the completion of the indefinite theta series $\Psi_{\mu}$. We determine furthermore the non-holomorphic completion of a closely related function $\Phi_{\mu}$ (4.58), which enumerates the number of "scaling" charge 
configurations. Although still involved, determination of the non-holomorphic completion of this function is simpler than for generating function of the BPS indices. The analysis of $\Phi_{\mu}$ and $\Psi_{\mu}$ demonstrates that these are mock modular forms of depth 2 . There are different representations of the completion:

1. As a non-holomorphic kernel of the theta series. This involves (generalized) error functions [36]. The modular properties of the completion follow from application of results by Vignéras [55]. This is applied to the partition functions for scaling black holes in section 4.4. For the partition function $\widehat{\Phi}_{\boldsymbol{\mu}}$ see equation (4.87), and for $\widehat{\Psi}_{\boldsymbol{\mu}}$, see equation (4.116).

2. Another useful representation, well-known for mock modular forms [34, 35], is as a holomorphic $q$-series plus an (iterated) integral of modular forms [36, 43, 56, 57]. This form is determined for three-center scaling black holes in equation (4.90) and (4.105). The modular transformations of the $q$-series follow directly from those of the iterated integral. This representation is also relevant physically, where the non-holomorphic part is contributed to continuum of states [58], or the Coulomb branch [44, 48].

3. The third representation we mention here is as an integral over a domain in a symmetric space, studied by Funke, Kudla and Millson [38, 39, 59], also known as Narain moduli space in the context of conformal field theory. In the case of a 3-center scaling solution, this would be a union of triangles in $\mathrm{SO}\left(2,2 b_{2}-2 ; \mathbb{Z}\right) \backslash \mathrm{SO}\left(2,2 b_{2}-\right.$ $2 ; \mathbb{R}) / \mathrm{SO}(2 ; \mathbb{R}) \times \mathrm{SO}\left(2 b_{2}-2 ; \mathbb{R}\right)$. While we have not explored this representation in detail, it is interesting to mention in light of recent discussion on averaging over Narian moduli space and the $\mathrm{AdS}_{3} / \mathrm{CFT}_{2}$ correspondence [60, 61].

Though technically involved, we believe that our results can be extended to scaling solutions with $n>3$ cores, and will give rise to mock modular forms of depth $n-1$. Any Calabi-Yau manifold with $b_{2} \geq 2$ gives rise to such mock modular forms, which thus provides a large resource of holomorphic higher depth mock modular forms. Further, it will be interesting to include single center degeneracies. Moreover, we hope that our results could be used for the study of $\mathrm{AdS}_{3}$ fragmention, and the interpretation of these solutions in the dual CFT. In this way, it may be possible to derive the non-holomorphic terms within gravity or the worldvolume theory of intersecting D-branes.

The outline of this paper is as follows. Section 2 reviews aspects of multi-center black holes, and in particular the index of scaling solutions. Section 3 reviews partition functions of D4-brane black holes. Section 4 discusses charge lattices for D4-brane bound states, and defines the partition functions of scaling black holes. We define here also the partition functions $\Phi_{\boldsymbol{\mu}}$ and $\Psi_{\boldsymbol{\mu}}$, which enumerate scaling configurations and their BPS indices, and determine its modular completion. Section 5 discusses the relation to M-theory and the decoupling limit $\mathrm{AdS}_{3}$. Section 6 considers two case studies for a specific Calabi-Yau 3-fold and charges, and presents the holomorphic $q$-series which are mock modular forms of depth 2 . 


\section{Black hole solutions in $\mathcal{N}=2$ supergravity}

We briefly review in this section supersymmetric black holes in $\mathcal{N}=2$ supergravity and partition functions.

\subsection{Black hole bound states}

Let $X$ be a simply connected Calabi-Yau threefold, with triple intersection product $d_{a b c}$, $a, b, c=1, \ldots, b_{2}$. The intersection product $d_{a b c}$ is symmetric in its indices. The classical central charge of a BPS state is given by

$$
Z(\gamma, t)=-\int_{X} e^{-t} \wedge \gamma
$$

where $\gamma$ on the r.h.s. is the Poincaré dual differential form of the homology class of the cycle which supports the D-branes, and is in 1-to-1 correspondence with the electric-magnetic charge of the BPS state. Moreover, $t$ is the Kähler modulus of the Calabi-Yau three-fold.

The scalar fields $X^{I}, I=0, \ldots, b_{2}$ of the vector multiplets are related to the Calabi-Yau moduli, $t^{a}=B^{a}+i J^{a}$, as $t^{a}=X^{a} / X^{0}$ for $a=1, \ldots, b_{2}$. Near the horizon, their values are determined by the attractor equations at the horizon in terms of the electric-magnetic charges of the black hole [62-64]. On the other hand, their asymptotic values for $|\vec{r}| \rightarrow \infty$ are boundary conditions for the equations of motion.

Besides the single center black hole, the equations of motion of $\mathcal{N}=2$ supergravity give rise to intricate multi-center black hole solutions $[3,6,65,66]$. Upon varying the asymptotic values of the scalar fields, multi-center solutions can cease to exist as proper solutions to the supergravity equations of motions, or reversely new solutions can appear. If the asymptotic values are chosen equal to the attractor values only few multi-center solutions exist. That is to say, only multi-center solutions which can be continuously connected to a single center black hole exist. These are the scaling solutions mentioned above, and are the main focus of this paper.

To understand this more explicitly, recall that an $n$-center solution, is required to satisfy the following $n-1$ Denef equations,

$$
\sum_{i \neq j} \frac{\left\langle\gamma_{i}, \gamma_{j}\right\rangle}{r_{i j}}=\left.2 \operatorname{Im}\left(e^{-\mathrm{i} \alpha} Z\left(\gamma_{i}, t\right)\right)\right|_{r=\infty}
$$

where $\langle$,$\rangle is the symplectic innerproduct between the charges,$

$$
\left\langle\gamma_{1}, \gamma_{2}\right\rangle=-P_{1}^{0} Q_{0,2}+P_{1} \cdot Q_{2}-P_{2} \cdot Q_{1}+P_{2}^{0} Q_{0,1} \cdot
$$

Moreover, $r_{i j}=\left|\vec{r}_{i}-\vec{r}_{j}\right|$ is the distance between the centers $i$ and $j$, and $\alpha$ is the phase of the central charge $Z(\gamma, t)$ for the total charge $\gamma=\sum_{i=1}^{n} \gamma_{i}$. We set

$$
c_{j}=\left.2 \operatorname{Im}\left(e^{-\mathrm{i} \alpha} Z\left(\gamma_{j}, t\right)\right)\right|_{r=\infty} .
$$

We fix $\vec{r}_{1}$ at the origin $\mathbb{R}^{3}$ and let $\mathcal{M}_{n}$ be the solution space for $\vec{r}_{j} \in \mathbb{R}^{3}, j=2, \ldots, n$ to (2.2). Then $\mathcal{M}_{n}$ has dimension $2 n-2$. The low energy degrees of freedom of the supersymmetric 
multi-center black hole give rise to $\mathcal{N}=4$ quiver quantum mechanics [65]. The quiver for an $n$-center bound state with charges $\left\{\gamma_{j}\right\}$ consists of $n$ nodes, and $\gamma_{i j}>0$ arrows from node $i$ to node $j$.

Note that the equations (2.2) are necessary but not sufficient for the multi-center solution to exist, to this end one needs to verify that the full supergravity solution is regular away from the black hole singularities, and without time-like curves [3]. Since we restrict to the large volume limit of the Calabi-Yau moduli space, we assume that this is the case in the following, and that we can determine the existence of bound states from (2.2).

The gravity perspective has led to the following form for the index of an $n$-center bound state $[6,9]$. To express this, we first introduce the rational index $\bar{\Omega}(\gamma)$ associated to the integer index $\Omega(\gamma)$,

$$
\bar{\Omega}(\gamma)=\sum_{m \mid \gamma} \frac{\Omega(\gamma / m)}{m^{2}} .
$$

The single center invariants $\Omega_{S}(\gamma)$ are the internal degeneracies of a supersymmetric particle or black hole with charge $\gamma$. It is expected to be a positive integer for a black hole. To analyze the spectrum of bound states it is convenient to introduce a fugacity $y$ for angular momentum. The rational variant of the refined index is defined as

$$
\bar{\Omega}(\gamma, y)=\sum_{m \mid \gamma} \frac{1}{m} \frac{y-y^{-1}}{y^{m}-y^{-m}} \Omega\left(\gamma / m, y^{m}\right) .
$$

This reproduces (2.5) in the limit $y \rightarrow 1$. A few variants of BPS indices will be important for us. We mention,

- The BPS index $\Omega(\gamma ; t)$, which enumerates BPS states for a given value $t$ of the asymptotic moduli. This includes single-center BPS states as well as bound states.

- The attractor index $\Omega\left(\gamma ; t_{\gamma}\right)$ with the moduli $t$ fixed at the attractor point determined by $\gamma$. These indices are the input for determining $\Omega(\gamma ; t)$ using attractor flow trees $[3$, $4,6,26,70]$.

- The single-center index $\Omega_{S}(\gamma)$, which is the internal degeneracy of a BPS particle or black hole center. This invariant is independent of the moduli $t$. These invariants are the input determining $\Omega(\gamma ; t)$ using the Coulomb branch formula [67, 68].

- The total invariant $\Omega_{T}(\gamma)$, which is a composite of $\Omega_{S}(\gamma)$ and independent of the moduli $t$. We give the expression below in (2.9).

The rational refined BPS index $\bar{\Omega}(\gamma, y ; t)$ can be expressed as a sum over partitions of $\gamma$, each representing a BPS bound state. It takes the form $[9,67]$

$$
\bar{\Omega}(\gamma, y ; t)=\sum_{\gamma=\sum_{i=1}^{n} \gamma_{i}} \frac{g_{C}\left(\left\{\gamma_{j}\right\},\left\{c_{j}\right\}, y\right)}{\left|\operatorname{Aut}\left(\left\{\gamma_{j}\right\}\right)\right|} \prod_{j=1}^{n} \bar{\Omega}_{T}\left(\gamma_{j}, y\right),
$$


with,

- $\left|\operatorname{Aut}\left(\left\{\gamma_{j}\right\}\right)\right|$ is the order of the subgroup of the permutation group, which preserves the ordered set $\left\{\gamma_{1}, \ldots, \gamma_{n}\right\}$.

- The index $g_{C}$ can be determined using localization of the black hole solution with respect to rotation around a fixed axis generated by $J_{3}$, say the $z$-axis [68]. A fixed point $p \in \mathcal{M}_{n}$ corresponds to a collinear solution with all centers placed on the $z$-axis. If the associated bound state quiver has no oriented loop, $g_{C}$ is the refined index of the $\mathcal{N}=4$ quiver quantum mechanics describing the bound state [9],

$$
g_{C}\left(\left\{\gamma_{j}\right\},\left\{c_{j}\right\}, y\right)=\operatorname{Tr}_{\mathcal{H}_{\mathrm{qm}}}^{\prime}(-y)^{2 J_{3}}
$$

where the trace is over the BPS Hilbert space $\mathcal{H}_{\mathrm{qm}}$ of the quiver quantum mechanics, and $J_{3}$ is one of the generators of $\mathrm{SU}(2)$.

- $\Omega_{T}$ the total invariant defined in terms of single centered indices $\Omega_{S}$ by

$$
\Omega_{T}(\gamma, y)=\Omega_{S}(\gamma, y)+\sum_{\sum_{j=1}^{n} m_{j} \gamma_{j}=\gamma} H\left(\left\{\gamma_{i}, m_{i}\right\}, y\right) \prod_{i=1}^{n} \Omega_{S}\left(\gamma_{i}, y^{m_{i}}\right),
$$

where $m_{j} \in \mathbb{N}$ are multiplicities of the charges in the partition of $\gamma$. For bound states whose associated quiver has no closed loops, the $H\left(\left\{\gamma_{i}, m_{i}\right\}, y\right)$ vanish. Otherwise they are determined by the "minimal modification hypothesis". This has the effect that if we express (2.7) as

$$
\bar{\Omega}(\gamma, y ; t)=\sum_{\gamma=\sum_{i=1}^{n} \gamma_{i}} \frac{\bar{g}_{C}\left(\left\{\gamma_{j}\right\},\left\{c_{j}\right\}, y\right)}{\left|\operatorname{Aut}\left(\left\{\gamma_{j}\right\}\right)\right|} \prod_{j=1}^{n} \bar{\Omega}_{S}\left(\gamma_{j}, y\right),
$$

then the $\bar{g}_{C}$ are $\mathrm{SU}(2)$ characters.

To determine $g_{C}$ using localization, it is convenient to introduce the refined index

$$
g_{C}\left(\left\{\gamma_{j}\right\},\left\{c_{j}\right\}, y\right)=\operatorname{Tr}_{\mathcal{H}_{\mathrm{qm}}}^{\prime}(-y)^{2 J_{3}}
$$

Let $z_{j}$ be the position of the center with charge $\gamma_{j}$. The localization technique then gives the following sum over collinear fixed points with respect to rotation around this axis,

$$
g_{C}\left(\left\{\gamma_{j}\right\},\left\{c_{j}\right\} ; y\right)=(-1)^{n-1}\left(y-y^{-1}\right)^{-n+1} \sum_{p \in \mathcal{M}_{n}} s(p)(-y)^{\sum_{i<j} \gamma_{i j} \operatorname{sgn}\left(z_{j}-z_{i}\right)},
$$

where $s(p) \in \pm 1$ is a sign depending on the details of the fixed point, and $z_{j}$ is the $z$ coordinate of center $j$. If the associated quiver does not contain loops, this is the complete index and the $y \rightarrow 1$ limit is well-defined. However, if the quiver contains loops, the distances between the black hole centers may be arbitrarily small [6]. Such solutions are known as scaling solutions, and additional fixed points need to be included in (2.11). An ( $n$-center) scaling black hole is a multi-center solution of $n$ black holes, whose phase space 
$\mathcal{M}_{n}$ contains a region where the centers can approach each other arbitrarily close. Thus, while the centers are spatially separated for generic points of $\mathcal{M}_{n}$, they are adiabatically connected to the black hole solution with a single center.

While many BPS bound states decay if we tune the moduli to their attractor values, scaling solutions remain part of the BPS spectrum. Since the index is evaluated at the attractor point $c_{j}^{*}$, each term on the r.h.s. of (2.7) with $n \geq 3$ corresponds to a scaling solution.

Various quantities may diverge in the limit $y \rightarrow 1$, such as $\Omega_{T}$ and $g_{C}$. In order to arrive at numerical counterparts for these quantities, we propose to regularize a rational function of the form

$$
\frac{f(y)}{\left(y-y^{-1}\right)^{\ell}}, \quad \text { with } \quad \lim _{y \rightarrow 1} f(y) \neq 0 \text {, }
$$

as follows

$$
\left.\frac{f(y)}{\left(y-y^{-1}\right)^{\ell}} \longrightarrow \frac{1}{2^{\ell} \ell !}\left(y \frac{d}{d y}\right)^{\ell} f(y)\right|_{y=1} .
$$

\subsection{Bound state indices}

Let us consider the equations (2.2) for small values of $n$. For $n=2$, there is a single equation,

$$
\frac{\gamma_{12}}{r_{12}}=c_{1}
$$

We deduce that the two-center solution only exists as a physical solution if $\gamma_{12} c_{1}>0$. This depends on the moduli $t$. If $t$ approaches a value where $c_{1}$ vanishes, $r_{12}$ diverges and the solution disappears as a solution to the supergravity equations of motion. In particular for the attractor point, the two-center solution never exists.

The quantum states of the two-center solution correspond to the product of the internal degeneracies of the centers times the states of a spin $\left(\left|\gamma_{12}\right|-1\right) / 2$ multiplet, which arises due to the electric-magnetic fields sourced by the charges of the two-centers [65]. We express it here as the product

$$
\Omega_{2}\left(\gamma_{1}+\gamma_{2} ; t\right)=g_{C}\left(\left\{\gamma_{1}, \gamma_{2}\right\},\left\{c_{1}, c_{2}\right\}\right) \Omega\left(\gamma_{1}\right) \Omega\left(\gamma_{2}\right),
$$

with

$$
g_{C}\left(\left\{\gamma_{1}, \gamma_{2}\right\},\left\{c_{1}, c_{2}\right\}\right)=\frac{1}{2}\left(\operatorname{sgn}\left(\gamma_{12}\right)+\operatorname{sgn}\left(c_{1}\right)\right)(-1)^{\gamma_{12}-1} \gamma_{12},
$$

and $\Omega\left(\gamma_{j}\right)$ are degeneracies of the individual centers. For sgn, we use the definition

$$
\operatorname{sgn}(x)=\left\{\begin{aligned}
1, & x>0 \\
0, & x=0 \\
-1, & x<0
\end{aligned}\right.
$$

The function $\frac{1}{2}\left(\operatorname{sgn}\left(\gamma_{12}\right)+\operatorname{sgn}\left(c_{1}\right)\right)$ equals 1 if the solution to $(2.14)$ is physical, i.e. $r_{12}>0$, and it vanishes if the sign of $r_{12}$ is unphysical, $r_{12}<0$. The factor $(-1)^{\gamma_{12}-1} \gamma_{12}$ is the number of states of the bound state, assuming that it exists. The case that $c_{1}=0$ is a special case, we aim to avoid. At the attractor point for the total charge, the $c_{j}(2.4)$ are equal to $c_{j}^{*}$, given by $[3,4]$

$$
c_{j}^{*}=\left|Z\left(\gamma, t_{\gamma}^{*}\right)\right|\left\langle\gamma, \gamma_{j}\right\rangle \text {. }
$$


As a result, two-center solutions do not exist at the attractor point, because substituting $c_{1}^{*}$ in (2.14) gives a negative value for $r_{12}$ which is unphysical.

For $n=3$ distinct charges, (2.2) gives two independent equations,

$$
\begin{aligned}
& \frac{\gamma_{12}}{r_{12}}+\frac{\gamma_{13}}{r_{13}}=c_{1}, \\
& \frac{\gamma_{21}}{r_{12}}+\frac{\gamma_{23}}{r_{23}}=c_{2} .
\end{aligned}
$$

An intriguing aspect of these equations is that for appropriate values of $\gamma_{i j}$, they can be satisfied with positive $r_{i j}$ for all pairs $i \neq j$, for $t$ at the attractor point. Then, there is a one-parameter family of solutions [6], with

$$
r_{i j}=\varepsilon \gamma_{i j}+O\left(\varepsilon^{2}\right), \quad \varepsilon \rightarrow 0,
$$

for $i j$ equal to 12,23 and 31.

We can in fact do better, and give an all order solution in $\varepsilon$. The parameter $\varepsilon$ together with three angular variables form the 4-dimensional solution space to (2.19). We set

$$
\gamma_{12}=a, \quad \gamma_{23}=b, \quad \gamma_{31}=c,
$$

in the following, and assume $a, b, c>0 .{ }^{1}$ We can then verify that the following 1-parameter family of distances $r_{i j}$ satisfy Denef's equations (2.2),

$$
\frac{1}{r_{12}}=\frac{1}{a \varepsilon}-\rho_{a}, \quad \frac{1}{r_{23}}=\frac{1}{b \varepsilon}-\rho_{b}, \quad \frac{1}{r_{31}}=\frac{1}{c \varepsilon}-\rho_{c},
$$

where $\rho_{a}, \rho_{b}, \rho_{c}$ satisfy $\left(\rho_{c} c-\rho_{a} a\right)=c_{1},\left(\rho_{a} a-\rho_{b} b\right)=c_{2},\left(\rho_{b} b-\rho_{c} c\right)=c_{3}$. The range of $\varepsilon$ is determined by triangle inequalities and positivity of $r_{12}, r_{23}, r_{31}$. The equations for $\rho_{a, b, c}$ allow for a shift, which modifies the range of $\varepsilon$ accordingly.

We discuss this now in detail for the attractor point, where we can use (2.18) such that $\left|Z\left(\gamma, t_{\gamma}^{*}\right)\right|=M$ and $\rho_{a}=\rho_{b}=\rho_{c}=M$. We then have,

$$
\frac{1}{r_{12}}=\frac{1}{a \varepsilon}-M, \quad \frac{1}{r_{23}}=\frac{1}{b \varepsilon}-M, \quad \frac{1}{r_{31}}=\frac{1}{c \varepsilon}-M .
$$

The free parameter $\varepsilon$ is bounded from below by 0 . In $\varepsilon \ll 1 / M$ regime, this solution reduces to eq. (2.20); thus existence of the scaling solution requires $a, b, c$ to obey triangle inequalities [6]. As $\varepsilon$ is increased, the shape of the triangle changes, and we need to determine the correct maximum of $\varepsilon$ domain. First, positivity of $r_{12}, r_{23}, r_{31}$ imposes the upper bound $\varepsilon \leq \frac{1}{M \max (a, b, c)}$. However, we also need to impose that $r_{i j}$ satisfy the triangle inequalities, which imposes an even stronger upper bound. E.g. the condition $r_{12}+r_{23} \geq r_{31}$ requires

$$
(a+b-c)-2 a b \varepsilon M+a b c \varepsilon^{2} M^{2} \geq 0 .
$$

If $(c-a)(c-b)<0$, this condition is always satisfied since $a+b-c>0$ and the l.h.s. does not have real roots in this case. Moreover if $(c-a)(c-b) \geq 0$, the condition

\footnotetext{
${ }^{1}$ We apologize for the multiple use of $a, b$ and $c$.
} 
is saturated for $\varepsilon_{c}^{ \pm}=\frac{1}{M c}\left[1 \pm \sqrt{\frac{(c-a)(c-b)}{a b}}\right]$ and violated for $\varepsilon_{c}^{-}<\varepsilon<\varepsilon_{c}^{+}$. Both roots are non-negative provided $(a, b, c)$ obeys the triangle inequality $a+b \geq c$. Noting that $\varepsilon_{c}^{+}>\frac{1}{M c}$, we see $\varepsilon \geq \varepsilon_{c}^{+}$makes $r_{31}$ negative. Thus we must have $\varepsilon \leq \varepsilon_{c}^{-}$. Using two other triangle inequalities, we have $\varepsilon \in\left(0, \min \left(\varepsilon_{a}^{-}, \varepsilon_{b}^{-}, \varepsilon_{c}^{-}\right) \mathbb{R}\right]$ where $\varepsilon_{a}^{-}=\frac{1}{M a}\left[1-\sqrt{\frac{(a-b)(a-c)}{b c}}\right]$, $\varepsilon_{b}^{-}=\frac{1}{M b}\left[1-\sqrt{\frac{(b-a)(b-c)}{a c}}\right]$, and $\min \left(\varepsilon_{a}^{-}, \varepsilon_{b}^{-}, \varepsilon_{c}^{-}\right)_{\mathbb{R}}$ means the minimum among the $\varepsilon_{a, b, c}^{-} \in \mathbb{R}$. One of $\varepsilon_{a, b, c}^{-}$may be complex. At the maximal value of $\varepsilon$ the configuration is collinear. With the three additional angular variables, one can aligned the solution along the $z$-axis, thus giving a fixed point for rotations around this axis.

For three centers, the sum over fixed points reads

$$
\begin{aligned}
g_{C}\left(\left\{\gamma_{j}\right\} ;\left\{c_{j}\right\} ; y\right)= & \frac{(-1)^{a+b+c}}{\left(y-y^{-1}\right)^{2}}\left[F(123) y^{a+b-c}+F(321) y^{-a-b+c}+F(213) y^{-a+b-c}\right. \\
& \left.+F(312) y^{a-b+c}+F(132) y^{a-b-c}+F(231) y^{-a+b+c}\right]
\end{aligned}
$$

where

$$
F(i j k):=F\left(i j k ;\left\{c_{j}\right\}\right)=\left\{\begin{aligned}
s(p), & \exists \text { a fixed point } p \in \mathcal{M}_{n} \text { with } z_{i}<z_{j}<z_{k}, \\
0, & \nexists \text { a fixed point } p \in \mathcal{M}_{n} \text { with } z_{i}<z_{j}<z_{k} .
\end{aligned}\right.
$$

Since $a, b, c \in \mathbb{Z}$, the signs $(-1)^{a \pm b \pm c}$ equal $(-1)^{a+b+c}$, and we can factor this out from the sum over permutations. The dependence on the r.h.s. on $\left\{c_{j}\right\}$ is through $\mathcal{M}_{n}$.

Since $F\left(123 ;\left\{c_{j}\right\}\right)=F\left(321 ;\left\{c_{j}\right\}\right)$, we can shorten $g_{C}$ to

$$
\begin{aligned}
g_{C}\left(\left\{\gamma_{j}\right\} ;\left\{c_{j}\right\} ; y\right)= & \frac{(-1)^{a+b+c}}{\left(y-y^{-1}\right)^{2}}\left[F\left(123 ;\left\{c_{j}\right\}\right)\left(y^{a+b-c}+y^{-a-b+c}\right)\right. \\
& \left.+F\left(213 ;\left\{c_{j}\right\}\right)\left(y^{-a+b-c}+y^{a-b+c}\right)+F\left(132 ;\left\{c_{j}\right\}\right)\left(y^{a-b-c}+y^{-a+b+c}\right)\right] .
\end{aligned}
$$

This expression does not have a smooth $y \rightarrow 1$ limit if $\mathcal{M}_{n}$ is non-compact and contains a scaling region. In that case, only one of $F\left(i j k ;\left\{c_{j}\right\}\right)$ 's is non-vanishing. Turning on a fugacity is indeed known to be subtle for non-compact phase spaces [69].

In the present context, the minimal modification hypothesis is put forward in [68] to correct this. It adds a term with minimal angular momentum corresponding to the fixed point with coincident centers. The effect of the minimal modification hypothesis for three distinct charges is that the refined index $g_{C}\left(\left\{\gamma_{j}\right\} ;\left\{c_{j}\right\}\right)$ is completed to

$$
\bar{g}_{C}\left(\left\{\gamma_{j}\right\} ;\left\{c_{j}\right\}, y\right)=g_{C}\left(\left\{\gamma_{j}\right\} ;\left\{c_{j}\right\}, y\right)+H\left(\left\{\gamma_{j}\right\}, y\right)
$$

with

$$
H\left(\left\{\gamma_{j}\right\}, y\right)= \begin{cases}-\frac{2}{\left(y-y^{-1}\right)^{2}}, & \text { if } a+b+c \in 2 \mathbb{Z} \\ \frac{y+y^{-1}}{\left(y-y^{-1}\right)^{2}}, & \text { if } a+b+c \in 2 \mathbb{Z}+1\end{cases}
$$


$\bar{g}_{C}$ has a well-defined $y \rightarrow 1$ limit, which reads,

$$
\begin{aligned}
\bar{g}_{C}\left(\left\{\gamma_{j}\right\} ;\left\{c_{j}\right\}\right) & =\lim _{y \rightarrow 1} \bar{g}_{C}\left(\left\{\gamma_{j}\right\} ;\left\{c_{j}\right\} ; y\right) \\
& =(-1)^{a+b-c}[F(123) s(a, b, c)+F(213) s(a, c, b)+F(132) s(c, b, a)],
\end{aligned}
$$

with

$$
s(a, b, c)=\left\{\begin{aligned}
\frac{(a+b-c)^{2}}{4}, & \text { if } a+b+c \in 2 \mathbb{Z}, \\
\frac{(a+b-c)^{2}-1}{4}, & \text { if } a+b+c \in 2 \mathbb{Z}+1 .
\end{aligned}\right.
$$

We note that for degenerate cases, where one or more among $a, b$ and $c$ vanish, $g_{C}$ can be a multiple of $1 / 2$ or $1 / 4$ rather than in $\{-1,0,1\}$.

Using the regularization (2.13), we obtaine for the numerical version (2.29) of $H$,

$$
H\left(\left\{\gamma_{j}\right\}\right)= \begin{cases}0, & \text { if } a+b+c \in 2 \mathbb{Z}, \\ \frac{1}{4}, & \text { if } a+b+c \in 2 \mathbb{Z}+1,\end{cases}
$$

which in turn gives the numerical counterpart for $\Omega_{T}$,

$$
\Omega_{T}(\gamma)=\Omega_{S}(\gamma)+\left\{\begin{aligned}
0, & \text { if } a+b+c \in 2 \mathbb{Z}, \\
\frac{1}{4} \prod_{j=1}^{3} \Omega_{S}\left(\gamma_{j}\right), & \text { if } a+b+c \in 2 \mathbb{Z}+1 .
\end{aligned}\right.
$$

We obtain similarly using (2.13) for the numerical version of $g_{C}$,

$$
\begin{aligned}
g_{C}\left(\left\{\gamma_{j}\right\} ;\left\{c_{j}\right\}\right)= & \frac{(-1)^{a+b+c}}{4} \times\left[F\left(123 ;\left\{c_{j}\right\}\right)(a+b-c)^{2}\right. \\
& \left.+F\left(213,\left\{c_{j}\right\}\right)(a-b+c)^{2}+F\left(132,\left\{c_{j}\right\}\right)(a-b-c)^{2}\right] .
\end{aligned}
$$

Thus the numerical $g_{C}$ essentially corresponds to the $y \rightarrow 1$ limit of the equivariant volume of the solution space $\mathcal{M}_{3}$ [68].

The term $F\left(123,\left\{c_{j}\right\}\right)$ is determined in [51, equation (2.57)]. For our purposes we rewrite this in terms of sgn rather than the step function. This reads,

$$
F\left(123,\left\{c_{j}\right\}\right)=F_{1}\left(a, b, c,\left\{c_{j}\right\}\right)+F_{2}(a, b, c),
$$

with

$$
\begin{aligned}
F_{1}\left(a, b, c,\left\{c_{j}\right\}\right) & =\frac{1}{4}\left(\operatorname{sgn}(a)+\operatorname{sgn}\left(c_{1}\right)\right)\left(\operatorname{sgn}(b)-\operatorname{sgn}\left(c_{3}\right)\right), \\
F_{2}(a, b, c) & =\frac{1}{4}(\operatorname{sgn}(a)+\operatorname{sgn}(b))(\operatorname{sgn}(a+b-c)-\operatorname{sgn}(a+b)) .
\end{aligned}
$$

At special charge configurations, where one or more arguments of the signs vanish, this may differ from [51, equation (2.57)]. In such cases, the $F_{j}$ can be a fraction rather than an integer. To deal properly with such cases, we include additional terms below in eqs. (2.42) and (2.45).

Our interest in this paper is the index at the attractor point, thus

$$
g_{C}\left(\left\{\gamma_{j}\right\} ;\left\{c_{j}^{*}\right\}\right)
$$


where $c_{j}^{*}(2.18)$ is $c_{j}$ evaluated at the attractor point $t_{\gamma}^{*}$. Then $F_{1}$ reads

$$
F_{1}(a, b, c)=\frac{1}{4}(\operatorname{sgn}(a)+\operatorname{sgn}(c-a))(\operatorname{sgn}(b)+\operatorname{sgn}(c-b)) .
$$

Assuming non-vanishing arguments of the sign functions, we can simplify the sum $F^{*}(123)=F_{1}+F_{2}$ using the identity $[70, \text { eq. (A.1) }]^{2}$

$$
\left(\operatorname{sgn}\left(x_{1}\right)+\operatorname{sgn}\left(x_{2}\right)\right) \operatorname{sgn}\left(x_{1}+x_{2}\right)-\operatorname{sgn}\left(x_{1}\right) \operatorname{sgn}\left(x_{2}\right)=1, \quad\left(x_{1}, x_{2}\right) \neq(0,0),
$$

to $F^{*}(123)=F_{1}(a, b, c)+F_{2}(a, b, c)[70$, eq. $(4.10)]$,

$F^{*}(123)=\frac{1}{4}(1+\operatorname{sgn}(a-c) \operatorname{sgn}(b-c)+\operatorname{sgn}(b-c) \operatorname{sgn}(c-a-b)+\operatorname{sgn}(c-a-b) \operatorname{sgn}(a-c))$.

We stress that this expression may differ from (2.35) if the arguments of some products of sgn's vanish. For example for $a=0, b=c=1, F(123)=0$, while $F^{*}(123)$ equals $\frac{1}{4}$.

For the other permutations, we also define

$$
\begin{aligned}
& F_{3}(a, b, c)=\frac{1}{4}(\operatorname{sgn}(a)+\operatorname{sgn}(b-a))(\operatorname{sgn}(c)+\operatorname{sgn}(b-c)), \\
& F_{4}(a, b, c)=\frac{1}{4}(\operatorname{sgn}(a)+\operatorname{sgn}(c))(\operatorname{sgn}(a+c-b)-\operatorname{sgn}(a+c)), \\
& F_{5}(a, b, c)=\frac{1}{4}(\operatorname{sgn}(c)+\operatorname{sgn}(a-c))(\operatorname{sgn}(b)+\operatorname{sgn}(a-b)), \\
& F_{6}(a, b, c)=\frac{1}{4}(\operatorname{sgn}(c)+\operatorname{sgn}(b))(\operatorname{sgn}(c+b-a)-\operatorname{sgn}(c+b)),
\end{aligned}
$$

and

$$
\begin{aligned}
& F^{*}(213)=\frac{1}{4}(1+\operatorname{sgn}(a-b) \operatorname{sgn}(c-b)+\operatorname{sgn}(c-b) \operatorname{sgn}(b-a-c)+\operatorname{sgn}(b-a-c) \operatorname{sgn}(a-b)), \\
& F^{*}(132)=\frac{1}{4}(1+\operatorname{sgn}(c-a) \operatorname{sgn}(b-a)+\operatorname{sgn}(b-a) \operatorname{sgn}(a-b-c)+\operatorname{sgn}(a-b-c) \operatorname{sgn}(c-a)) .
\end{aligned}
$$

Having defined the $F^{*}(i j k)$, we turn to $g_{C}\left(\left\{\gamma_{j}\right\} ;\left\{c_{j}^{*}\right\}\right)$ and take care of the special cases where both arguments of a products of sgn's vanish. Let us first determine for which values of $a, b$ and $c, g_{C}\left(\left\{\gamma_{j}\right\} ;\left\{c_{j}^{*}\right\}\right)$ is affected. When the last two products of sgn's of $F^{*}(123)$ (2.40) vanish, the angular momentum factor $(a+b-c)^{2} / 4$ also vanishes. Thus replacing these products of sgn's with a non-vanishing value will not affect $g_{C}$. For the remaining product, $\operatorname{sgn}(a-c) \operatorname{sgn}(b-c)$ 's, the arguments vanish in the equilateral case $a=b=c$ for which the angular momentum factor is $a^{2} / 4$. This is the same as for the other permutations, $F^{*}(213)$ and $F^{*}(132)$, such that we can take all three into account by adding a single additional term with yet undetermined coefficient $A$. We obtain thus for $g_{C}$ at the attractor point,

$$
\begin{aligned}
g_{C}\left(\left\{\gamma_{j}\right\} ;\left\{c_{j}^{*}\right\}\right)= & \frac{(-1)^{a+b+c}}{4}\left[F^{*}(123)(a+b-c)^{2}+F^{*}(213)(a-b+c)^{2}\right. \\
& \left.+F^{*}(132)(-a+b+c)^{2}+\frac{1}{4} A \delta_{a, c} \delta_{b, c} a^{2}\right] .
\end{aligned}
$$

\footnotetext{
${ }^{2}$ We thank Sergey Alexandrov for stressing the simplifying power of this identity.
} 
At this point, we can make a "guess" of the preferred "physical" value for $A$. From the gravity perspective, the equilateral configurations with $a=b=c$ are perfectly fine multicenter solutions, such that it is most natural that these contribute with $1 \times(-1)^{a} a^{2} / 4$ to $g_{C}$. For $(2.42)$, we have instead $(3+A) \times(-1)^{a} a^{2} / 16$, thus suggesting $A=1$. We will demonstrate in section 4.5 that modularity of the $q$-series leads to exactly the same value.

With this we arrive at our final expression for the numerical attractor index $\Omega\left(\gamma ; t_{\gamma}\right)$ with $\gamma$ partitioned into at most three constituent charges is given by,

$$
\bar{\Omega}\left(\gamma ; t_{\gamma}\right)=\Omega_{T}(\gamma)+\sum_{\gamma=\sum_{i=1}^{3} \gamma_{i}} g_{C}\left(\left\{\gamma_{j}\right\} ;\left\{c_{j}^{*}\right\}\right) \prod_{j=1}^{3} \bar{\Omega}_{T}\left(\gamma_{j}\right),
$$

with $g_{C}\left(\left\{\gamma_{j}\right\} ;\left\{c_{j}^{*}\right\}\right)$ given by $(2.42)$, and $\Omega_{T}(\gamma)$ given in terms of $\Omega_{S}$ by (2.33).

Besides the bound state index $g_{C}$, we are also interested to enumerate the number of charge configurations giving rise to scaling black holes. Up to vanishing arguments, the sum $F_{\text {total }}(a, b, c)=\sum_{j=1}^{6} F_{j}(a, b, c)$ can be further simplified to

$$
\begin{aligned}
F_{\text {total }}(a, b, c)= & \frac{1}{4}[1+\operatorname{sgn}(a+b-c) \operatorname{sgn}(a+c-b) \\
& +\operatorname{sgn}(a+c-b) \operatorname{sgn}(b+c-a)+\operatorname{sgn}(b+c-a) \operatorname{sgn}(a+b-c)] .
\end{aligned}
$$

This expression has the advantage that for $a+b+c$ odd, the arguments of the sgn's never vanish. With inclusion of additional terms to deal with vanishing arguments, we define number $f_{C}$,

$$
\begin{aligned}
f_{C}\left(\left\{\gamma_{j}\right\},\left\{c_{j}^{*}\right\}\right)= & F_{\text {total }}(a, b, c)(-1)^{a+b+c} \\
& +\frac{1}{4} A_{1} \delta_{a, 0} \delta_{b, c}+\frac{1}{4} A_{2} \delta_{c, 0} \delta_{a, b}+\frac{1}{4} A_{3} \delta_{b, 0} \delta_{a, c},
\end{aligned}
$$

where the constants $A_{j}$ are yet to be determined. We will determine these from the modular completion. To our surprise, these numbers are typically irrational for $a+b+c \in 2 \mathbb{Z}$, while they do not contribute for $a+b+c \in 2 \mathbb{Z}+1$. We find the irrationality quite peculiar. On the other hand, $f_{C}$ is not a BPS index, such that it is not really violating any physical principles.

\section{Review of D4-brane black holes}

We review in this section aspects of partition functions of D4-brane black holes. We start in subsection 5 by reviewing the uplift of D4-brane black holes to M-theory. In subsection 3.1, we discuss the "supergravity" partition function, which enumerates D4-brane BPS indices $\Omega(\gamma ; t)$ for fixed Kähler modulus $t$. In subsection 3.2, we discuss the "attractor" partition function, which is a generating function of BPS indices $\Omega\left(\gamma ; t_{\gamma}^{*}\right)$ evaluated at the attractor point $t_{\gamma}^{*}$ of the corresponding charge $\gamma$.

\subsection{Supergravity partition function}

From the perspective of asymptotically flat $\mathbb{R}^{4}$, the most natural BPS partition function is enumerates the BPS indices for a fixed, asymptotic value of the (Kähler) moduli $t$, and in 
the mixed ensemble where the magnetic charge $P$ is fixed $[6,13,14,71]$ and the electric charge $Q$ is varied. The electric charge takes value in the lattice $\Lambda \simeq \mathbb{Z}^{b_{2}}$ with bi-linear form $D_{a b}=d_{a b c} P^{c}$. For a positive magnetic charge $P^{a}, d_{a b c} P^{c}$ provides a non-degenerate quadratic form with signature $\left(1, b_{2}-1\right)$ for the lattice $\Lambda$ of magnetic charges. The electric charges $Q_{a}$ take values in dual lattice $\Lambda^{*}$, with quadratic form $D^{a b}=\left(d_{a b c} P^{c}\right)^{-1}$. We abbreviate the pairing between an element $Q \in \Lambda^{*}$ and $k \in \Lambda$ as

$$
\sum_{a=1}^{b_{2}} Q_{a} P^{a}=Q . P
$$

and extend this by linearity in each argument to $\Lambda^{*} \otimes \mathbb{R}$ and $\Lambda \otimes \mathbb{R}$. For later use, we also introduce the notation $\mu \in \Lambda^{*} / \Lambda$,

$$
\Lambda_{\mu}^{*}=\{Q \in \Lambda+\mu+P / 2\} .
$$

We stress that the elements of $\Lambda_{\mu}^{*}$ do not necessarily lie in $\Lambda^{*}$ due to the shift by $P / 2$. Using this notation, the partition function for D4-D2-D0 black holes reads schematically

$$
\mathcal{Z}_{S G}(\tau, C, t)=\sum_{Q_{0}, Q_{a}} \bar{\Omega}(\gamma, t)(-1)^{P \cdot Q} e^{-2 \pi \tau_{2}|Z(\gamma, t)|+2 \pi i \tau_{1}\left(Q_{0}-Q . B+B^{2} / 2\right)+2 \pi i C \cdot(Q-B / 2)},
$$

where $\bar{\Omega}(\gamma, t)$ is the rational index $(2.5), \tau_{1} \in \mathbb{R}$ is the $\mathrm{RR} 1$-form $C_{1}$, and $\tau_{2}=e^{-\phi} \in \mathbb{R}_{+}$ with $\phi$ being the dilaton, $C$ the RR 3 -form and $B$ the $B$-field.

Here $Z(\gamma, t)$ is the central charge of the $\mathcal{N}=2$ algebra. Ignoring the non-perturbative terms in the strict large volume limit, $Z$ reads

$$
Z(\gamma, t)=\frac{1}{2} P \cdot\left(J^{2}-B^{2}\right)+Q \cdot B-Q_{0}+i(Q-B P) \cdot J .
$$

The BPS mass becomes in this limit,

$$
|Z(\gamma, t)|=\frac{1}{2} P .\left(J^{2}-B^{2}\right)+Q . B-Q_{0}+\frac{((Q-B P) . J)^{2}}{P . J^{2}},
$$

up to inverse powers of $J$. By assumption $J^{2}>0$, hence $J^{a}$ lies in the positive cone of $\Lambda$. Thus $\frac{k . J J}{J^{2}}$ is the component of the vector $k$ along $J$.

In the large volume limit, supergravity has an $\operatorname{Sp}\left(2 b_{2}+2, \mathbb{Z}\right)$ symmetry, generated by matrices

$$
\mathbb{K}(k)=\left(\begin{array}{cccc}
1 & 0 & 0 & 0 \\
k^{a} & \mathbb{I}_{b_{2}} & 0 & 0 \\
\frac{1}{2} d_{a b c} k^{b} k^{c} & d_{a b c} k^{c} & \mathbb{I}_{b_{2}} & 0 \\
\frac{1}{6} d_{a b c} k^{a} k^{b} k^{c} & \frac{1}{2} d_{a b c} k^{b} k^{c} & k^{a} & 1
\end{array}\right), k \in \mathbb{Z}^{b_{2}},
$$

and acts linearly on $\gamma=\left(P^{0}, P^{a}, Q_{a}, Q_{0}\right)$. For $P^{0}=0$, these transformations preserve the magnetic charge, and act on remaining charges and moduli as follows:

$$
\begin{aligned}
Q_{a} & \rightarrow Q_{a}+d_{a b c} k^{b} P^{c}, \\
Q_{0} & \rightarrow Q_{0}+k^{a} Q_{a}+\frac{1}{2} d_{a b c} k^{a} k^{b} P^{c}, \\
t^{a} & \rightarrow t^{a}+k^{a} .
\end{aligned}
$$


We introduce the abbreviations:

$$
\begin{aligned}
\hat{Q}_{\overline{0}} & :=-Q_{0}+\frac{1}{2} Q^{2}, \\
\hat{Q} & :=Q-B, \\
\hat{Q}_{-}^{2} & :=\hat{Q}^{2}-\hat{Q}_{+}^{2}, \\
(Q-B)_{+}^{2} & :=\frac{((Q-B J) \cdot P)^{2}}{P J^{2}} .
\end{aligned}
$$

Note that the combinations $\hat{Q}, \hat{Q}_{\overline{0}}$ and the conjugacy class $\mu$ of electric charge vector are invariant under the transformations (3.7). Invariance of the conjugacy class is seen by noting that spectral flow shifts $Q$ by integer lattice vectors, when mapped to magnetic lattice $\Lambda$ and such shifts do not change the conjugacy class.

The holomorphic and anti-holomorphic dependence on $\tau$ can be made more manifest, if we rewrite $\mathcal{Z}_{S G}$ as

$$
\mathcal{Z}_{S G}(\tau, C, t)=e^{-\pi \tau_{2} J^{2}} \sum_{Q_{0}, Q} \bar{\Omega}\left(P, Q, Q_{0} ; t\right)(-1)^{P \cdot Q} \bar{q}^{\hat{Q}_{\overline{0}}-\hat{Q}_{-}^{2} / 2} q^{\hat{Q}_{+}^{2} / 2} e^{2 i \pi C \cdot(Q-B / 2)},
$$

where $q=e^{2 \pi i \tau}$. The transformations (3.7) act on $\mathcal{Z}_{S G}$ as

$$
\mathcal{Z}_{S G}(\tau, C, t) \rightarrow(-1)^{P . k} e^{\pi i C . k} \mathcal{Z}_{S G}(\tau, C, t),
$$

under the action of $\mathbb{K}(k)$.

We can map the system of D4-D2-D0 branes to IIB string theory, by a T-duality along time circle. The RR 1-form $C_{1}$ is mapped to the RR 0-form $C_{0}$, while the D4, D2 and D0-branes respectively become D3, D1 branes and D(-1)-instantons. Moreover, the action of the IIB strong-weak duality on the instantonic branes carries over to the spectrum of D4-D2-D0 branes in IIA string theory. The duality acts as follows. The modular parameter is $\tau=C_{1}+i e^{-\phi}$, with $\phi$ being the dilaton and $C_{1}$ the Ramond-Ramond 1-form flux along $S_{t}^{1}$. For an element $\left(\begin{array}{ll}a & b \\ c & d\end{array}\right) \in \mathrm{SL}(2, \mathbb{Z})$. This duality acts as

$$
\begin{aligned}
\tau & \rightarrow \frac{a \tau+b}{c \tau+d}, \\
\left(\begin{array}{l}
C \\
B
\end{array}\right) & \rightarrow\left(\begin{array}{ll}
a & b \\
c & d
\end{array}\right)\left(\begin{array}{l}
C \\
B
\end{array}\right), \\
J & \rightarrow|c \tau+d| J,
\end{aligned}
$$

In a series of papers $[6,13,14,18,25,26,29]$, the supergravity partition function (3.3) has been analyzed. We summarize the main properties:

- Quasi-periodicity in the two-form fields $B$ and $C$.

For $k \in \Lambda$, we have

$$
\begin{aligned}
& \mathcal{Z}_{S G}(\tau, C, t+k)=(-1)^{P . k} e^{\pi i C . k} \mathcal{Z}_{S G}(\tau, C, t), \\
& \mathcal{Z}_{S G}(\tau, C+k, t)=(-1)^{P . k} e^{-\pi i B . k} \mathcal{Z}_{S G}(\tau, C, t) .
\end{aligned}
$$

These can be seen as large gauge transformations. 
- $\mathrm{SL}(2, \mathbb{Z})$ S-duality:

$$
\begin{aligned}
S: & \mathcal{Z}_{S G}(-1 / \tau,-B, C+|\tau| J) & =\tau^{1 / 2} \bar{\tau}^{-3 / 2} \varepsilon(S) \mathcal{Z}_{S G}(\tau, C, t), \\
T: & \mathcal{Z}_{S G}(\tau+1, C+B, t) & =\varepsilon(T) \mathcal{Z}_{S G}(\tau, C, t),
\end{aligned}
$$

where $\varepsilon(S)=\varepsilon(T)^{-3}, \varepsilon(T)=e^{-i \pi \frac{c_{2}(X) . P}{12}}$ are phases. The factor $\bar{\tau}^{-3 / 2}$ can be understood from the non-compact bosons in the CFT due to the center of mass in $\mathbb{R}^{3}$.

- The partition function involves an intricate dependence on the moduli $t$ through wall-crossing. The proper modular invariant partition function differs from (3.9) by additional non-holomorphic, subleading terms which are non-holomorphic in $\tau$.

\subsection{Attractor partition function}

The supergravity partition function is a rather complicated function. It has become clear that an alternative partition function is a more amenable object to study [18]. This is the attractor partititon function $\mathcal{Z}_{P}^{\lambda}$, which is the generating function of indices $\bar{\Omega}\left(\gamma ; t_{\gamma}^{\lambda}\right)$, where each index is evaluated at its (large volume) attractor point $t_{\gamma}^{\lambda}$. The indices $\bar{\Omega}\left(\gamma ; t_{\gamma}^{\lambda}\right)$ were also referred to $\Omega_{\text {CFT }}$ in [25], and MSW invariants $\Omega^{\mathrm{MSW}}$ in [18]. For irreducible magnetic charge $P$ and magnetic charges which can be written as a sum of at most 2 magnetic charges, these indices are conjectured to coincide with the CFT indices. However as discussed in section 5, our findings in this paper suggest that there is a difference for generic magnetic charges due to scaling black holes.

This partition function is obtained by replacing $\Omega(\gamma, t)$ in the definition of $\mathcal{Z}_{S G}$ by its attractor value $\Omega\left(\gamma, t_{\gamma}^{\lambda}\right)$. For a D4-D2-D0 black hole with charge $\gamma=\left(0, P^{a}, Q_{a}, Q_{0}\right)$, we have for the "large volume" attractor value $\left(t_{\gamma}^{*}\right)^{a}$,

$$
\left(t_{\gamma}^{\lambda}\right)^{a}=D^{a b} Q_{b}+i \lambda P^{a}
$$

with $\lambda$ sufficiently large, such that subleading terms in a $\lambda$ expansion can be ignored. Eq. (3.14) is equivalent to

$$
B_{\gamma}^{a}=D^{a b} Q_{b}, \quad\left(J_{\gamma}^{\lambda}\right)^{a}=\lambda P^{a}
$$

The precise proportionality constant $\lambda$ between $J^{a}$ and $P^{a}$ will not be important for us, since we will restrict to the large volume limit, $|J| \gg 1$. On the other hand, we do not take the limit $\lambda \rightarrow \infty$, since physical quantities such as the BPS mass diverge in this limit.

The attractor or MSW partition function then reads,

$$
\mathcal{Z}_{P}^{\lambda}(\tau, C, t)=\sum_{Q_{0}, Q_{a}} \bar{\Omega}\left(\gamma, t_{\gamma}^{\lambda}\right)(-1)^{P \cdot Q} e^{-2 \pi \tau_{2}|Z(\gamma, t)|+2 \pi i \tau_{1}\left(Q_{0}-Q . B+B^{2} / 2\right)+2 \pi i C \cdot(Q-B / 2)}
$$

Note that although the degeneracy is evaluated at the attractor point $t_{\gamma}^{\lambda}$, the mass in the exponent is the ADM mass evaluated for moduli at infinity being $t$. The other moduli dependence in the exponent is also similar to that of $\mathcal{Z}_{S G}(\tau, C, t)$. 
Since black holes states contributing to the attractor index exist everywhere in moduli space, their quantum degeneracies should be moduli independent. Moreover, one can show that $\Omega\left(\gamma ; t_{\gamma}^{\lambda}\right)$ are invariant under spectral flow transformations (3.7). This entails that $\Omega\left(\gamma ; t_{\gamma}^{\lambda}\right)$ depends on the charges only through the invariant combination $\hat{Q}_{\overline{0}}$ and the conjugacy class $\mu$. These imply the sum

$$
h_{P, \mu}(\tau):=\sum_{Q_{0}} \bar{\Omega}\left(\gamma ; t_{\gamma}^{\lambda}\right) q^{\hat{Q}_{\overline{0}}},
$$

for fixed $Q$ is also invariant under spectral flow transformations and apart from the magnetic charge, depends solely on the conjugacy class $\mu$. It has been understood from wallcrossing and the perspective of D-instantons, that $h_{P, \mu}$ receives additional non-holomorphic contributions $h_{P, \mu}[18,29]$. The resultant is the non-holomorphic function $\widehat{h}_{P, \mu}(\tau, \bar{\tau})$.

Hence one is led to the following theta function decomposition of the attractor partition function,

$$
\begin{aligned}
\mathcal{Z}_{P}^{\lambda}(\tau, C, t) & =e^{-\pi \tau_{2} J^{2}} \sum_{\mu \in \Lambda^{*} / \Lambda} \overline{h_{P, \mu}(\tau)} \Theta_{\mu}(\tau, \bar{\tau}, C, B), \\
\text { where } \Theta_{\mu}(\tau, \bar{\tau}, C, B) & =\sum_{Q \in \Lambda_{\mu}^{*}}(-1)^{P . Q} q^{\hat{Q}_{+}^{2} / 2} \bar{q}^{-\hat{Q}_{-}^{2} / 2} e^{2 \pi i C .(Q-B / 2)},
\end{aligned}
$$

with the set $\Lambda_{\mu}^{*}$ is given in (3.2). We define analogously the completion $\widehat{\mathcal{Z}}_{P}^{\lambda}$ by replacing $h_{P, \mu}$ by $\widehat{h}_{P, \mu}$ in (3.18).

Various factors in the right hand side of (3.18) also have definite modular transformation properties. The prefactor $e^{-\pi \tau_{2} J^{2}}$ is modular invariant. Moreover, the Siegel-Narain theta function $\Theta_{\mu}(\tau, C, B)$ transforms as

$$
\begin{aligned}
S: \quad \Theta_{\mu}(-1 / \tau,-1 / \bar{\tau},-B, C)= & \frac{1}{\sqrt{\left|\Lambda^{*} / \Lambda\right|}}(-i \tau)^{b_{2}^{+} / 2}(i \bar{\tau})^{b_{2}^{-} / 2} e^{-i \pi P^{2} / 2} \\
& \times \sum_{\nu \in \Lambda^{*} / \Lambda} e^{-2 \pi i \mu . \nu} \Theta_{\nu}(\tau, \bar{\tau}, C, B), \\
T: \quad \Theta_{\mu}(\tau+1, \bar{\tau}+1, C+B, B)= & e^{i \pi(\mu+P / 2)^{2}} \Theta_{\mu}(\tau, \bar{\tau}, B, C) .
\end{aligned}
$$

This implies that the appropriate completion $\widehat{h}_{P, \mu}$ transforms as a vector valued modular form,

$$
\begin{aligned}
& S: \quad \widehat{h}_{P, \mu}(-1 / \tau,-1 / \bar{\tau})=-\frac{1}{\sqrt{\left|\Lambda^{*} / \Lambda\right|}}(-i \tau)^{-b_{2} / 2-1} \varepsilon(S)^{*} e^{-i \pi P^{2} / 2} \sum_{\delta \in \Lambda^{*} / \Lambda} e^{-2 \pi i \delta . \mu} \widehat{h}_{P, \delta}(\tau, \bar{\tau}), \\
& T: \quad \widehat{h}_{P, \mu}(\tau+1, \bar{\tau}+1)=\varepsilon(T)^{*} e^{i \pi(\mu+P / 2)^{2}} \widehat{h}_{P, \mu}(\tau, \bar{\tau}),
\end{aligned}
$$

where $\varepsilon(S)$ and $\varepsilon(T)$ are as below (3.13).

\section{Partition functions for scaling black holes}

Our aim in this section is determine and analyze the first multi-center contribution to the attractor partition function (3.17) with the moduli at the large volume attractor points 
$t_{\gamma}^{\lambda}$ (3.14). The large volume attractor points are in many ways analogous to the proper attractor points $t_{\gamma}$. For this value of the moduli and assuming that $\gamma$ and $\gamma_{j}$ both carry D4-brane charge, the $c_{j}(2.4)$ evaluated at $t_{\gamma}^{\lambda}$ are to leading order equal to $c_{j}^{\lambda}$,

$$
c_{j}^{\lambda}=2 \lambda\left\langle\gamma, \gamma_{j}\right\rangle
$$

Since the factor $2 \lambda$ on the r.h.s. is positive, the large volume attractor point is similar in nature to the $c_{j}^{*}(2.18)$ for the proper attractor point.

On the other hand, specific bound state solutions may exist at $t_{\gamma}^{\lambda}$ which are unstable at $t_{\gamma}$. Examples of such bound states are pairs of D6 and anti-D6 branes. The spatial separation of these such bounds state is of the order $\ell_{5}^{3} / R^{2}$, which decreases as $\lambda^{-3}$ in the large $\lambda$ limit. On the other hand, the separation of centers that carry D4-brane charge, but vanishing D6 charge scales as (4.1), scales as $\lambda^{-1}$ except for the near coincident region. From the perspective of the large volume limit, the (D6, anti-D6) bound states are effectively single centers. To emphasize that they are bound states with a small separation, we introduce the notion of "core" for a black hole solution with separation of the order $\ell_{5}^{3} / R^{2}$ between the centers. The discussion of section 2 is easily adapted to the large volume regime by reading "single core" for "single center". One naturally defines a single core index $\Omega_{S C}$ and total core invariant $\Omega_{T C}$ analogous to the single center index $\Omega_{S C}$ and total invariant $\Omega_{T C}$.

The first multi-center, or multi-core, contribution to the attractor partition function (3.17) consists of bound states with three cores, which each carry a positive D4-brane charge and vanishing D6-brane charge. The symplectic innerproducts $a, b$ and $c(2.21)$ read in this case,

$$
\begin{aligned}
& a=P_{1} Q_{2}-P_{2} Q_{1}, \\
& b=P_{2} Q_{3}-P_{3} Q_{2}, \\
& c=P_{3} Q_{1}-P_{1} Q_{3} .
\end{aligned}
$$

This gives for the $c_{j}^{\lambda}, c_{1}^{\lambda}=2 \lambda(c-a), c_{2}^{\lambda}=2 \lambda(a-b)$. Since the $c_{j}^{*}(2.18)$ and $c_{j}^{\lambda}(4.1)$ are simply related by the substitution $\left|Z\left(\gamma ; t_{\gamma}^{*}\right)\right| \rightarrow 2 \lambda$, the discussion on the solution to Denef's equations below (2.23) is applicable, and in particular gives the separation of collinear solutions.

At the attractor point $t_{\gamma}^{\lambda}$ many multi-center and multi-core configurations do not exist as physical solutions. However we have seen that some multi-center solutions do exist. These solutions are distinguished from generic solutions, since the centers can approach each other arbitrarily close. The scaling solutions do respect the spectral flow symmetry, since the symplectic innerproducts are invariant under (3.7).

We note that the existence scaling solutions poses constraints on the magnetic charges $P_{j}$. For example since for a scaling solution $a, b, c$ must have the same sign, we deduce that there are no scaling solutions for $P_{1}=P_{2}=P_{3}=P / 3$, since this gives rise to $\gamma_{12}+\gamma_{23}+\gamma_{31}=a+b+c=0$. Therefore, there must be an asymmetry in the magnetic charge of the centers for such scaling solutions to exist. To see that $F$ vanishes in the case of three equal magnetic charges, note that the second factors in $F_{2}, F_{4}, F_{6}$ vanish immediately as $\operatorname{sgn}(2 x)-\operatorname{sgn}(x)=0$. Moreover, in $F_{1}$ we can replace $c=-a-b$ and when $a>0$ the 
first factor imposes the constraint $-2 a-b \geq 0$ so $b<-2 a$ and $b$ must be negative. Hence the second factor in $F_{1}$ vanishes. The argument goes similarly for $F_{3}, F_{5}$. Similarly one may show that such scaling black holes only exist for $b_{2}>1$.

\subsection{Bound states and lattices}

We discuss in this subsection various aspects of charge lattices of bound states, and the projection of the charge lattice to a sublattice with fixed total charge. The discussion in this subsection does not rely on the existence of scaling black holes for these charges and lattices.

Lattice decomposition. We apply some techniques of decompositions and gluings of integral lattices to charge lattices of bound states. See for example [72, 73]. We will consider a black hole bound state of $n$ centers, with non-vanishing, positive magnetic charge $P_{j} \in \Lambda_{j}$, $j=1,2, \ldots, n$, where $\Lambda_{j}$ is the $b_{2}$-dimensional lattice associated to the $j$ 'th center with innerproduct $D_{j}$,

$$
D_{j a b}=d_{a b c} P_{j}^{c}, \quad a, b, c=1, \ldots, b_{2} .
$$

The total magnetic charge is $P=\sum_{j=1}^{n} P_{j}$ with quadratic form $D$ and $b_{2}$-dimensional lattice $\Lambda$. The signature $\Lambda_{j}$ and $\Lambda$ is $\left(1, b_{2}-1\right)$.

We use boldface notation for the lattices for boundstates. For $n$ centers, we introduce $\left(n b_{2}\right)$-dimensional lattices and vectors,

$$
\begin{aligned}
& \vec{k}=\left(k_{1}, k_{2}, \ldots, k_{n}\right) \in \boldsymbol{\Lambda}:=\Lambda_{1} \oplus \Lambda_{2} \oplus \cdots \oplus \Lambda_{n} \\
& \vec{x}=\left(x_{1}, x_{2}, \ldots, x_{n}\right) \in \Lambda^{*}:=\Lambda_{1}^{*} \oplus \Lambda_{2}^{*} \oplus \cdots \oplus \Lambda_{n}^{*}, \\
& \vec{Q}=\left(Q_{1}, Q_{2}, \ldots, Q_{n}\right) \in \Lambda^{*}+\vec{P} / 2=\Lambda_{1}^{*} \oplus \Lambda_{2}^{*} \oplus \cdots \oplus \Lambda_{n}^{*}+\left(P_{1}, P_{2}, \ldots, P_{n}\right) / 2 .
\end{aligned}
$$

We denote the quadratic form for $\boldsymbol{\Lambda}$ by $\vec{D}=\operatorname{diag}\left(D_{1}, D_{2}, \ldots, D_{n}\right)$.

Since we typically sum over bound states with fixed total electric charge, we aim to decompose the lattice $\boldsymbol{\Lambda}$ in a $b_{2}$-dimensional sublattice $\overline{\boldsymbol{\Lambda}} \subset \boldsymbol{\Lambda}$ representing the total charge, and its orthogonal complement $\underline{\boldsymbol{\Lambda}}$ with dimension $(n-1) b_{2}$ representing the relative charge distribution over the constituents. To introduce this properly, let $\overline{\boldsymbol{\Lambda}}$ be the sublattice $\bar{\Lambda} \subset \boldsymbol{\Lambda}$,

$$
\overline{\mathbf{\Lambda}}=\left\{\vec{k}=(k, k, \ldots, k) \in \boldsymbol{\Lambda} \mid k \in \mathbb{Z}^{b_{2}}\right\} .
$$

The lattice $\boldsymbol{\Lambda}$ induces a quadratic form on $\bar{\Lambda}$. Namely for $\vec{k} \in \boldsymbol{\Lambda}, \vec{D}(\vec{k})=\sum_{j=1}^{n} D_{j}(k)=D(k)$. For $k \in \mathbb{Z}^{b_{2}}$, this is the quadratic form for the total charge $P$ as desired. As a result, we have the group isomorphism $\Lambda^{*} / \Lambda=\bar{\Lambda}^{*} / \bar{\Lambda}$.

Let $\bar{\pi}$ be the orthogonal projection,

$$
\bar{\pi}: \Lambda \rightarrow \bar{\Lambda} \otimes \mathbb{Q}
$$

For $\vec{k} \in \boldsymbol{\Lambda}$ and $\vec{m} \in \bar{\Lambda}$, we have

$$
\vec{D}(\bar{\pi}(\vec{k}), \vec{m})=\vec{D}(\vec{k}, \bar{\pi}(\vec{m}))=\vec{D}(\vec{k}, \vec{m}) \in \mathbb{Z} .
$$

Therefore, $\bar{\pi}$ gives an injection of $\boldsymbol{\Lambda}$ to the dual lattice $\overline{\boldsymbol{\Lambda}}^{*}$,

$$
\bar{\pi}: \Lambda \rightarrow \bar{\Lambda}^{*}
$$


Moreover, if we extend $\bar{\pi}$ by linearity to $\boldsymbol{\Lambda}^{*}$, a similar argument to (4.7) shows that $\bar{\pi}: \boldsymbol{\Lambda}^{*} \rightarrow \overline{\boldsymbol{\Lambda}}^{*}$. The projection $\bar{\pi}$ is explicitly given for $\vec{k}=\left(k_{1}, k_{2}, \ldots, k_{n}\right) \in \boldsymbol{\Lambda}$ by,

$$
\bar{\pi}(\vec{k})=(k, k, \ldots, k) \in(\bar{\Lambda})^{*} \quad \text { with } \quad k=D^{-1} \sum_{j=1}^{n} D_{j} k_{j} .
$$

We define furthermore the kernel $\underline{\boldsymbol{\Lambda}}=\operatorname{Ker}(\bar{\pi})$,

$$
\underline{\boldsymbol{\Lambda}}:=\left\{\vec{k} \in \mathbf{\Lambda} \mid \sum_{j=1}^{n} D_{j} k_{j}=0\right\} .
$$

Elements in $\underline{\boldsymbol{\Lambda}}$ have vanishing innerproduct with $\overline{\boldsymbol{\Lambda}}$, such that they are indeed each others orthogonal complement in $\boldsymbol{\Lambda}$.

Since $\bar{\pi}(\vec{k}) \in \bar{\Lambda}$ for $\vec{k} \in \boldsymbol{\Lambda}$, we have that $\vec{k}-\bar{\pi}(\vec{k}) \in \underline{\boldsymbol{\Lambda}}$. Thus $\overline{\boldsymbol{\Lambda}} \oplus \underline{\boldsymbol{\Lambda}}$ is in the kernel of the homomorphism $\bar{h}: \boldsymbol{\Lambda} \rightarrow \overline{\boldsymbol{\Lambda}}^{*} / \overline{\boldsymbol{\Lambda}}$. We call

$$
G=\Lambda /(\bar{\Lambda} \oplus \underline{\Lambda})
$$

the glue group for the decomposition of $\boldsymbol{\Lambda}$, and the image of $G$ under $\bar{h}, \bar{h}(G) \subset \overline{\boldsymbol{\Lambda}}^{*} / \overline{\boldsymbol{\Lambda}}$, the glue group for $\overline{\boldsymbol{\Lambda}}$ [72]. The homomorphism $\bar{h}$ gives an injection of $G$ to the subgroup $\bar{h}(G) \subset \overline{\boldsymbol{\Lambda}}^{*} / \overline{\boldsymbol{\Lambda}}$. Therefore, the number of glue vectors, $N_{g}=|\boldsymbol{\Lambda} /(\overline{\boldsymbol{\Lambda}} \oplus \underline{\boldsymbol{\Lambda}})|$ is a factor in $\left|\overline{\boldsymbol{\Lambda}}^{*} / \overline{\boldsymbol{\Lambda}}\right|=\operatorname{det}(\bar{D})$. In the special case that $\operatorname{det}(\bar{D})$ is prime, or more generally co-prime with $\operatorname{det}\left(D_{j}\right)$ for all $j, N_{g}=\operatorname{det}(\bar{D})$. By the same arguments, there is a projection $\underline{\pi}: \boldsymbol{\Lambda} \rightarrow \underline{\boldsymbol{\Lambda}}^{*}$, and a homomorphism $\underline{h}: G \rightarrow \underline{\boldsymbol{\Lambda}}^{*} / \underline{\boldsymbol{\Lambda}}$. Therefore, $N_{g}$ is also a factor $\operatorname{in} \operatorname{det}(\underline{D})$. This gives for the number of glue vectors $N_{g}$ in general

$$
N_{g}=\sqrt{\frac{\operatorname{det}(\bar{D}) \operatorname{det}(\underline{D})}{\prod_{j=1}^{n} \operatorname{det}\left(D_{j}\right)}}
$$

The order of the quotient group $\left(\underline{\boldsymbol{\Lambda}}^{*} / \underline{\boldsymbol{\Lambda}}\right) / \underline{h}(G)$ is

$$
N_{q}=\frac{\operatorname{det}(\underline{D})}{N_{g}} .
$$

This will be useful for us in the following way. If we consider the class of vectors $\vec{k} \in \boldsymbol{\Lambda}$ with fixed projection to $\bar{\Lambda}^{*}$, for example $\bar{\pi}(\vec{k})=0$, this fixes an element of the glue group $G$, and thus also of the images of $G$ in $\bar{\Lambda}^{*} / \bar{\Lambda}$ and $\underline{\Lambda}^{*} / \underline{\Lambda}$. As a result, the number of possible conjugacy classes of $\underline{\pi}(\vec{k}) \in \underline{\boldsymbol{\Lambda}}^{*} / \underline{\boldsymbol{\Lambda}}$ of $\underline{\pi}(\vec{k})$ is $N_{q}$ (4.13). This is also the case for a vector $\vec{x} \in \boldsymbol{\Lambda}^{*}$ with fixed projection $\bar{\pi}(\vec{x}) \in \bar{\Lambda}^{*}$.

Similarly to $\Lambda_{\mu}^{*}(3.2)$, we introduce the notation $\underline{\Lambda}_{\mu}^{*}$,

$$
\underline{\Lambda}_{\mu}^{*}:=\left\{\vec{Q}=\left(Q_{1}, Q_{2}, \ldots, Q_{n}\right) \in \boldsymbol{\Lambda}+\left(\mu_{1}, \mu_{2}, \ldots, \mu_{n}\right)+\vec{P} / 2 \mid \sum_{j=1}^{n} Q_{j}=\mu+P / 2\right\},
$$


where the subscript $\boldsymbol{\mu} \in \underline{\Lambda}^{*} / \underline{\boldsymbol{\Lambda}}, \mu=\sum_{j=1}^{n} \mu_{j} \in \Lambda^{*}$ and $P=\sum_{j=1}^{n} P_{j} \in \Lambda$. We define the quadratic form $Q^{2}$ for $\underline{\Lambda}_{\mu}^{*}$,

$$
\begin{aligned}
\boldsymbol{Q}^{2} & =-Q^{2}+\sum_{j=1}^{3}\left(Q_{j}\right)_{j}^{2} \\
& =-(\mu+P / 2)^{2}+\sum_{j=1}^{3}\left(Q_{j}\right)_{j}^{2} .
\end{aligned}
$$

This form of the quadratic form appears naturally, when we determine the partition function $h_{\mu}^{s}$ (4.53) of scaling solutions in the next subsection. On the other hand, it should match with the inverse of the quadratic form for $\underline{\boldsymbol{\Lambda}}$. In the cases considered below, we find that this is indeed the case.

Note that in components, $P$ in (4.14) has a lower index and equals $P_{a}=d_{a b c} P^{b} P^{c}=$ $d_{a b c}\left(\sum_{j} P_{j}\right)^{b}\left(\sum_{j} P_{j}\right)^{c}$. To understand constraint $\sum_{j} Q_{j}=\mu+P / 2$ better, we change variables from $Q_{j} \in \Lambda_{j}^{*}$ to $k_{j} \in \Lambda_{j}$ using

$$
\begin{aligned}
Q_{1, a} & =\mu_{1, a}+D_{1 a b}\left(k_{1}^{b}+P_{2}^{b}+P_{1}^{b} / 2\right), \\
Q_{2, a} & =\mu_{2, a}+D_{2 a b}\left(k_{2}^{b}+P_{3}^{b}+P_{2}^{b} / 2\right), \\
& \vdots \\
Q_{n-1, a} & =\mu_{n-1, a}+D_{(n-1) a b}\left(k_{n}^{b}+P_{n}^{b}+P_{n-1}^{b} / 2\right), \\
Q_{n, a} & =\mu_{n, a}+D_{n a b}\left(k_{n}^{b}+P_{1}^{b}+P_{n}^{b} / 2\right),
\end{aligned}
$$

with $k_{j}^{b} \in \mathbb{Z}^{b_{2}}$ for $j=1, \ldots, n$. The shifts of $k_{j}^{b}$ by $P_{j}^{b}$ are included such that the required identity for the $k_{j}$ takes a compact form,

$$
\sum_{j=1}^{n} D_{j a b} k_{j}^{b}=0, \quad k_{j} \in \mathbb{Z}^{b_{2}},
$$

which is indeed identical to the defining condition for the lattice $\underline{\boldsymbol{\Lambda}}$ in (4.10). Solving this relation over the integers, $k_{j} \in \mathbb{Z}^{b_{2}}$, is in general a complicated problem depending on the $D_{j}$. Solving say for $k_{n}$, we have

$$
k_{n}=-D_{n}^{-1} \sum_{j=1}^{n-1} D_{j} k_{j} .
$$

Thus we find that if $D_{n}^{-1} D_{j}, j=1, \ldots n-1$ are not integral matrices, not all $k_{j} \in \mathbb{Z}^{b_{2}}$ can correspond to bound state charges for these conjugacy classes. In some cases, this problem can be avoid by solving for another $k_{j}$ instead of $k_{n}$, but not in general. We restrict to special cases in the following.

2- and 3-center bound states for $\boldsymbol{b}_{\mathbf{2}}=\mathbf{1}$. For $b_{2}=1$, the $D_{j}$ are simply positive numbers. For $n=2$, the relation (4.17) becomes

$$
D_{1} k_{1}+D_{2} k_{2}=0
$$


The solutions with $k_{1,2} \in \mathbb{Z}$ are

$$
k_{1}=\frac{D_{2}}{\operatorname{gcd}\left(D_{1}, D_{2}\right)} m, \quad k_{2}=-\frac{D_{1}}{\operatorname{gcd}\left(D_{1}, D_{2}\right)} m, \quad m \in \mathbb{Z},
$$

where gcd stands for the greatest common divisor. Substituting (4.20) in the quadratic form $\sum_{j=1,2} D_{j} k_{j}^{2}$, gives for the quadratic form $\underline{D}$ on $\underline{\boldsymbol{\Lambda}}$,

$$
\underline{D}=\frac{D_{1} D_{2}\left(D_{1}+D_{2}\right)}{\operatorname{gcd}\left(D_{1}, D_{2}\right)^{2}} .
$$

Thus the number of glue vectors (4.12) and order $N_{q}$ (4.13) are in this case,

$$
n=2: \quad N_{g}=\frac{D_{1}+D_{2}}{\operatorname{gcd}\left(D_{1}, D_{2}\right)}, \quad N_{q}=\frac{D_{1} D_{2}}{\operatorname{gcd}\left(D_{1}, D_{2}\right)} .
$$

Moving on to $n=3$, we need to find the integral solutions to

$$
D_{1} k_{1}+D_{2} k_{2}+D_{3} k_{3}=0
$$

To this end, we first consider $k_{3}=0$. Then, the solutions are obviously given by (4.20). Next for a fixed non-vanishing $k_{3}$, there are only solutions if $\operatorname{gcd}\left(D_{1}, D_{2}\right)$ divides $D_{3} k_{3}$ by Bézout's identity. As a result, if we choose $\operatorname{gcd}\left(D_{1}, D_{2}\right) / \operatorname{gcd}\left(D_{1}, D_{2}, D_{3}\right)$ for $k_{3}$, Bézout's identity asserts that there is an integral solution $\left(\ell_{1}, \ell_{2}\right)$ for $\left(k_{1}, k_{2}\right)$,

$$
D_{1} \ell_{1}+D_{2} \ell_{2}+D_{3} \frac{\operatorname{gcd}\left(D_{1}, D_{2}\right)}{\operatorname{gcd}\left(D_{1}, D_{2}, D_{3}\right)}=0
$$

Since this choice for $k_{3}$ has the smallest non-vanishing magnitude with integral solutions, the other choices for $k_{3}$ follow by multiplication by an integer $m_{1}$. Including also the solutions (4.20), we find for the general solution,

$$
\begin{aligned}
& k_{1}=\ell_{1} m_{1}+\frac{D_{2}}{\operatorname{gcd}\left(D_{1}, D_{2}\right)} m_{2}, \\
& k_{2}=\ell_{2} m_{1}-\frac{D_{1}}{\operatorname{gcd}\left(D_{1}, D_{2}\right)} m_{2}, \\
& k_{3}=\frac{\operatorname{gcd}\left(D_{1}, D_{2}\right)}{\operatorname{gcd}\left(D_{1}, D_{2}, D_{3}\right)} m_{1} .
\end{aligned}
$$

Substitution of (4.25) in $\sum_{j=1}^{3} D_{j} k_{j}^{2}$, one finds for the 2-dimensional quadratic form $\underline{D}$ for $\left(m_{1}, m_{2}\right)$,

$$
\underline{D}=\left(\begin{array}{cr}
D_{1} \ell_{1}^{2}+D_{2} \ell_{2}^{2}+D_{3} \frac{\operatorname{gcd}\left(D_{1}, D_{2}\right)^{2}}{\operatorname{gcd}\left(D_{1}, D_{2}, D_{3}\right)^{2}} & \frac{D_{1} D_{2}\left(\ell_{1}-\ell_{2}\right)}{\operatorname{gcd}\left(D_{1}, D_{2}\right)} \\
\frac{D_{1} D_{2}\left(\ell_{1}-\ell_{2}\right)}{\operatorname{gcd}\left(D_{1}, D_{2}\right)} & \frac{D_{1} D_{2}\left(D_{1}+D_{2}\right)}{\operatorname{gcd}\left(D_{1}, D_{2}\right)^{2}}
\end{array}\right)
$$

Using (4.24), we find for its determinant,

$$
\operatorname{det}(\underline{D})=\frac{D_{1} D_{2} D_{3}\left(D_{1}+D_{2}+D_{3}\right)}{\operatorname{gcd}\left(D_{1}, D_{2}, D_{3}\right)^{2}},
$$


which is symmetric in the $D_{j}$. For the number of glue vectors $N_{g}$ and order $N_{q}$, we now obtain,

$$
n=3: \quad N_{g}=\frac{D_{1}+D_{2}+D_{3}}{\operatorname{gcd}\left(D_{1}, D_{2}, D_{3}\right)}, \quad N_{q}=\frac{D_{1} D_{2} D_{3}}{\operatorname{gcd}\left(D_{1}, D_{2}, D_{3}\right)} .
$$

Together with the discussion below, this is very suggestive that the generalization to $n>3$ is

$$
\operatorname{det}(\underline{D})=\frac{\left(\sum_{j=1}^{n} D_{j}\right) \prod_{j=1}^{n} D_{j}}{\operatorname{gcd}\left(D_{1}, D_{2}, \ldots, D_{n}\right)^{2}}
$$

2- and 3-center bound states for $b_{2} \geq 1$ with simplifications. We continue to discuss the general case $b_{2} \geq 1$. Let us first consider $n=2$. We make the assumption that $D_{2}^{-1} D_{1}$ is an integral matrix. The equation (4.17) can be solved by setting $k_{2}=-D_{2}^{-1} D_{1} k_{1}$. Substituting this in the quadratic form $\vec{D}$, gives us for the $b_{2}$-dimensional quadratic form on $\underline{\Lambda}$

$$
\underline{D}=D_{1}+D_{1} D_{2}^{-1} D_{1} .
$$

This agrees with (4.21) upon specialization to $b_{2}=1$. We find then for $N_{g}$ and $N_{q}$,

$$
n=2: \quad N_{g}=\operatorname{det}\left(D_{2}^{-1} D\right), \quad N_{q}=\operatorname{det}\left(D_{1}\right) .
$$

To deal with our main case, $n=3$, we make two technical simplifications:

1. We assume that $D_{3}^{-1} D_{1}$ and $D_{3}^{-1} D_{2}$ are integral matrices, such that $k_{3} \in \mathbb{Z}^{b_{2}}$ in (4.18) if $k_{1}$ and $k_{2} \in \mathbb{Z}^{2}$.

2. We assume that $d_{a b c} \in 2 \mathbb{Z}$ for all $a, b, c$. Then $\Lambda^{*}+P / 2=\Lambda^{*}$ for any $P$ such that shifts by $P$ (and $P_{j}$ ) in (4.14), (4.15) and (4.16) are unnecessary.

These assumptions are satisfied in the examples in section 6 .

If we substitute now (4.18) in $\boldsymbol{Q}^{2}$, we arrive at

$$
\begin{aligned}
\boldsymbol{Q}^{2}= & -\mu^{2}+\left(\mu_{1}\right)_{1}^{2}+\left(\mu_{2}\right)_{2}^{2}+\left(\mu_{3}\right)_{3}^{2}+2\left(\mu_{1}-D_{3}^{-1} D_{1} \mu_{3}\right) \cdot k_{1}+2\left(\mu_{2}-D_{3}^{-1} D_{2} \mu_{3}\right) \cdot k_{2} \\
& +\left(k_{1}, k_{2}\right) \underline{D}\left(k_{1}, k_{2}\right)^{T},
\end{aligned}
$$

where $\underline{D}$ is the quadratic form of the lattice $\underline{\boldsymbol{\Lambda}}$,

$$
\underline{D}=\left(\begin{array}{cc}
D_{1}+D_{1} D_{3}^{-1} D_{1} & D_{1} D_{3}^{-1} D_{2} \\
D_{2} D_{3}^{-1} D_{1} & D_{2}+D_{2} D_{3}^{-1} D_{2}
\end{array}\right) .
$$

The determinant of $\underline{D}$ is given $b^{3}$

$$
\begin{aligned}
\operatorname{det}(\underline{D}) & =\operatorname{det}\left(D_{1}\right) \operatorname{det}\left(D_{2}\right) \operatorname{det}\left(D_{3}^{-1}\right) \operatorname{det}\left(D_{1}+D_{2}+D_{3}\right) \\
& =\operatorname{det}\left(D_{1}\right) \operatorname{det}\left(D_{2}\right) \operatorname{det}\left(D_{3}^{-1} D\right) .
\end{aligned}
$$

We have for $N_{g}$ and $N_{q}$ in this case,

$$
N_{g}=\operatorname{det}\left(D_{3}^{-1} D\right), \quad N_{q}=\operatorname{det}\left(D_{1}\right) \operatorname{det}\left(D_{2}\right)
$$

\footnotetext{
${ }^{3}$ We use that for an invertible $n \times n$ matrix $A$ and $n \times m$ matrices $U$ and $V$, we have $\operatorname{det}\left(A+U V^{T}\right)=$ $\operatorname{det}(A) \operatorname{det}\left(1_{m}+V^{T} A^{-1} U\right)$, https://en.wikipedia.org/wiki/Matrix_determinant_lemma.
} 
These formulas are in agreement with (4.27) for $b_{2}=1$, and $\operatorname{gcd}\left(D_{1}, D_{2}, D_{3}\right)=D_{3}$. Moreover for generic $b_{2}$, we can consider cases where we can solve (4.17) in terms of $k_{2}$ as well as $k_{3}$, and we expect a symmetry in $D_{2} \leftrightarrow D_{3}$. Indeed, then $D_{2}^{-1} D_{1}$ and $D_{2}^{-1} D_{3}$ are integral matrices too. The only way for $D_{2}^{-1} D_{3}$ and $D_{3}^{-1} D_{2}$ both to be integral is to satisfy $\left|\operatorname{det}\left(D_{2}\right)\right|=$ $\left|\operatorname{det}\left(D_{3}\right)\right|$, in which case (4.34) reduces to $\operatorname{det} \underline{D}= \pm \operatorname{det}\left(D_{1}\right) \operatorname{det}\left(D_{1}+D_{2}+D_{3}\right)$, which is symmetric under the exchange of 2 and 3 . Similar comments hold of course as well for $k_{1}$.

Using general formula's for inverses of block matrices [74], we derive that the inverse of $\underline{D}$ reads

$$
\begin{aligned}
\underline{D}^{-1} & =\left(\begin{array}{cc}
D^{-1}\left(D_{2}+D_{3}\right) D_{1}^{-1} & -D^{-1} \\
-D^{-1} & D^{-1}\left(D_{1}+D_{3}\right) D_{2}^{-1}
\end{array}\right) \\
& =\left(\begin{array}{cc}
D_{1}^{-1}-D^{-1} & -D^{-1} \\
-D^{-1} & D_{2}^{-1}-D^{-1}
\end{array}\right),
\end{aligned}
$$

One can verify that the determinant of $\underline{D}^{-1}$ is indeed the inverse of (4.34). Indeed, if we introduce the two components $\boldsymbol{\mu}_{1}$ and $\boldsymbol{\mu}_{2}$,

$$
\begin{aligned}
& \boldsymbol{\mu}_{1}=\mu_{1}-D_{1} D_{3}^{-1} \mu_{3}, \\
& \boldsymbol{\mu}_{2}=\mu_{2}-D_{2} D_{3}^{-1} \mu_{3},
\end{aligned}
$$

the quadratic form $\boldsymbol{Q}^{2}(4.32)$ for $k_{1}=k_{2}=0, \boldsymbol{Q}^{2}=\boldsymbol{\mu}^{2}$, can be written as

$$
\boldsymbol{\mu}^{2}=-\left(\boldsymbol{\mu}_{1}+\boldsymbol{\mu}_{2}\right)^{2}+\left(\boldsymbol{\mu}_{1}\right)_{1}^{2}+\left(\boldsymbol{\mu}_{2}\right)_{2}^{2}=\left(\boldsymbol{\mu}_{1}, \boldsymbol{\mu}_{2}\right) \underline{D}^{-1}\left(\boldsymbol{\mu}_{1}, \boldsymbol{\mu}_{2}\right)^{T} .
$$

For $k_{1}, k_{2}$ non-zero, we have $\boldsymbol{Q}=\left(\boldsymbol{Q}_{1}, \boldsymbol{Q}_{2}\right)$ with

$$
\begin{aligned}
& \boldsymbol{Q}_{1}=\mu_{1}-D_{1} D_{3}^{-1} \mu_{3}+\left(D_{1}+D_{1} D_{3}^{-1} D_{1}\right) k_{1}+D_{1} D_{3}^{-1} D_{2} k_{2}, \\
& \boldsymbol{Q}_{2}=\mu_{2}-D_{2} D_{3}^{-1} \mu_{3}+D_{2} D_{3}^{-1} D_{1} k_{1}+\left(D_{2}+D_{2} D_{3}^{-1} D_{2}\right) k_{2},
\end{aligned}
$$

or equivalently

$$
\begin{aligned}
& \boldsymbol{Q}_{1}=Q_{1}-D_{1} D_{3}^{-1} Q_{3}, \\
& \boldsymbol{Q}_{2}=Q_{2}-D_{2} D_{3}^{-1} Q_{3},
\end{aligned}
$$

which we can write more compactly as $\boldsymbol{Q}=\boldsymbol{\mu}+\underline{D} \boldsymbol{k}$ with $\boldsymbol{k}=\left(\boldsymbol{k}_{1}, \boldsymbol{k}_{2}\right)^{T}$. Thus an element $\boldsymbol{\mu} \in \underline{\boldsymbol{\Lambda}}^{*} / \underline{\boldsymbol{\Lambda}}$ is completely determined by

$$
\boldsymbol{\mu}=\left\{\left(\mu_{1}, \mu_{2}, \mu_{3}, \mu\right) \mid \mu_{j} \in \Lambda_{j}^{*}, \quad \mu_{1}+\mu_{2}+\mu_{3}=\mu \in \Lambda^{*}\right\} .
$$

Generic number of constituents (with simplifications). The analysis for a generic number $n$ of constituents (including $n=2$ ) follows analogously. The quadratic form reads as in (4.15), but with 3 replaced by $n$ in the summation, and with constraint $\sum_{j=1}^{n} Q_{j}=\mu+P / 2$. Similarly to (4.17), the constraint can be expressed as

$$
\sum_{j=1}^{n} D_{j a b} k_{j}^{b}=0 .
$$


With the assumption that $D_{n}^{-1} D_{j}$ is an integer matrix for all $j=1, \ldots, n$, this can be solved and the quadratic form becomes

$$
\underline{D}=\operatorname{diag}\left(D_{1}, \ldots, D_{n-1}\right)+\left(\begin{array}{c}
D_{1} \\
\ldots \\
D_{n-1}
\end{array}\right)\left(D_{n}^{-1} D_{1}, \ldots, D_{n}^{-1} D_{n-1}\right),
$$

with inverse

$$
\underline{D}^{-1}=\operatorname{diag}\left(D_{1}^{-1}, \ldots, D_{n-1}^{-1}\right)-\left(\begin{array}{c}
D^{-1} \\
\ldots \\
D^{-1}
\end{array}\right)(1, \ldots, 1),
$$

where $D=\sum_{j=1}^{n} D_{j}$. Moreover, the determinant of $\underline{D}$ is

$$
\begin{aligned}
\operatorname{det}(\underline{D}) & =\operatorname{det}\left(D_{n}^{-1}\right) \operatorname{det}(D) \prod_{j=1}^{n-1} \operatorname{det}\left(D_{j}\right) \\
& =\operatorname{det}\left(D_{n}^{-1} D\right) \prod_{j=1}^{n-1} \operatorname{det}\left(D_{j}\right),
\end{aligned}
$$

from which $N_{g}$ and $N_{q}$ are easily determined.

Characteristic vectors. We briefly discuss here a characteristic vector for the lattice $\underline{\Lambda}$ with $n=3$, which is important for the theta series of the scaling solutions. A sign which frequently occured in section 2 is $(-1)^{a+b+c}$. Such signs in a theta series are typically written in terms of a characteristic vector. We therefore express $a+b+c$ as

$$
a+b+c=\boldsymbol{K} \cdot \boldsymbol{Q}
$$

with

$$
\boldsymbol{K}=\left(P_{3}-P_{2}, P_{1}-P_{3}\right) .
$$

This is a characteristic vector of $\underline{\boldsymbol{\Lambda}}$. Indeed, we have with $\vec{P}=\left(P_{1}, P_{2}, P_{3}\right)$,

$$
\vec{k} \cdot \vec{P}+\vec{k}^{2} \in 2 \mathbb{Z}
$$

since $P_{j}$ is a characteristic vector for $\Lambda_{j}, j=1,2,3$. We can decompose with respect to the lattice decomposition $\underline{\boldsymbol{\Lambda}} \oplus \overline{\boldsymbol{\Lambda}}$,

$$
\boldsymbol{k} \cdot \boldsymbol{K}+\boldsymbol{k}^{2}+k \cdot P+k^{2} .
$$

Since $P$ is a characteristic vector for $\Lambda$, this shows that $\boldsymbol{K}$ is a characteristic vector for $\boldsymbol{\Lambda}$.

However, if we express $a+b+c+P . Q$ in terms of vectors in $\Lambda=\sum_{j} \Lambda_{j}$, such that $a+b+c=\vec{K} \cdot \vec{Q} \bmod 2$, then $\vec{K}=\left(P_{3}-P_{2}, P_{1}-P_{3}, P_{2}-P_{1}\right)$. Then $(\vec{K}+\vec{P}) \cdot \vec{Q}=$ $\boldsymbol{K} \cdot \boldsymbol{Q}+\vec{P} \cdot \vec{Q}=P \cdot Q \bmod 2$. Moreover, $\vec{P}$ is a characteristic vector of $\boldsymbol{\Lambda}$, and

$$
\vec{P}^{2}=P^{3}+\boldsymbol{K}^{3} \bmod 4 .
$$




\subsection{Partition functions}

We consider black hole bound states with three cores. The $j^{\text {th }}$ core carries electric and magnetic charges $Q_{j}$ and $P_{j}$ respectively. It is natural to work with a mixed ensemble with total magnetic charge $P$ held fixed. For the present purpose, we shall fix the total electric D2-brane charge $Q=\sum_{j} Q_{j} \in \Lambda^{*}+P / 2$ with $\mu \in \Lambda^{*} / \Lambda$ as well. We work at the attractor value of the moduli, corresponding to total charge vector $(P, Q)$. At this point apart from single core black holes, the only other black holes to survive are the scaling black holes. A natural question is - for a fixed total charge, how many scaling black holes are there and what is their contribution to the index?

In analogy to the attractor indices $\bar{\Omega}\left(\gamma, t_{\gamma}^{\lambda}\right)$, we define the generating function $h_{\mu}^{T}(\tau)$ of numerical total core invariants $\bar{\Omega}_{T C}$ introduced earlier in this section,

$$
h_{P, \mu}^{T}(\tau)=\sum_{Q_{0}} \bar{\Omega}_{T C}(\gamma) q^{\hat{Q}_{\overline{0}}}
$$

We can similarly define the partition function of single core indices $h_{P, \mu}^{S}(\tau)$, with $\Omega_{T}$ replaced by $\Omega_{S}$. The $\Omega_{T}(\gamma)$ are determined from the refined ones (2.9) using the regularization (2.13). For the 3 -core case, this gives (2.33). If $P$ is irreducible, i.e. it can not be written as a sum of more than 1 positive magnetic charge, and the three partition functions agree.

Based on (2.7), we can express the attractor partition function $h_{P, \mu}$ in terms of the partition function $h_{P, \mu}^{T}$. We have schematically

$$
h_{P, \mu}(\tau)=h_{P, \mu}^{T}(\tau)+\sum_{n>1} \sum_{\substack{\sum_{j=1}^{n} P_{j}=P \\ \sum_{j=1}^{n} Q_{j}=Q}} \frac{g_{C}\left(\left\{\gamma_{j}\right\},\left\{c_{j}^{\lambda}\right\}\right)}{\left|\operatorname{Aut}\left(\left\{\gamma_{j}\right\}\right)\right|} q^{\mu^{2} / 2-\sum_{j}\left(Q_{j}\right)_{j}^{2} / 2} \prod_{j=1}^{n} h_{P_{j}, \mu_{j}}^{T}(\tau) .
$$

Recall that there are no 2-center/core scaling black holes, such that there is no contribution from $n=2$ on the r.h.s.

We will proceed by considering the term in (4.52) with $n=3$. Using the notation introduced in section 4.1, we can enumerate the number of three-core scaling black holes as

$$
h_{\left\{P_{j}\right\}, \mu}^{3 T}(\tau)=\sum_{\substack{\mu_{j} \in \Lambda_{j}^{*} / \Lambda_{j}, j=1,2,3 \\ \mu_{1}+\mu_{2}+\mu_{3}=\mu}} h_{P_{1}, \mu_{1}}^{T}(\tau) h_{P_{2}, \mu_{2}}^{T}(\tau) h_{P_{3}, \mu_{3}}^{T}(\tau) \Psi_{\mu}(\tau),
$$

with $\boldsymbol{\mu}$ as in (4.41), and where $\Psi_{\boldsymbol{\mu}}$ is the indefinite theta series

$$
\Psi_{\boldsymbol{\mu}}(\tau)=\sum_{\boldsymbol{Q} \in \underline{\boldsymbol{\Lambda}}_{\mu}^{*}} g_{C}\left(\left\{\gamma_{j}\right\},\left\{c_{j}^{\lambda}\right\}\right) q^{-Q^{2} / 2} .
$$

With $y=e^{2 \pi i z}$, we define the refined series as

$$
\Psi_{\boldsymbol{\mu}}(\tau, z)=\left(y-y^{-1}\right)^{2} \sum_{\boldsymbol{Q} \in \underline{\boldsymbol{\Lambda}}_{\boldsymbol{\mu}}^{*}} g_{C}\left(\left\{\gamma_{j}\right\},\left\{c_{j}^{\lambda}\right\} ; y\right) q^{-Q^{2} / 2},
$$

with $g_{C}\left(\left\{\gamma_{j}\right\},\left\{c_{j}^{\lambda}\right\}\right)$ as in $(2.42)$ and $g_{C}\left(\left\{\gamma_{j}\right\},\left\{c_{j}^{\lambda}\right\} ; y\right)$ as in (2.27). Note that $\Psi_{\boldsymbol{\mu}}$ is symmetric as function of $z$,

$$
\Psi_{\mu}(\tau,-z)=\Psi_{\mu}(\tau, z)
$$


The two functions are related by (2.13),

$$
\Psi_{\mu}(\tau)=\left.\left(\frac{1}{4 \pi i} \frac{\partial}{\partial z}\right)^{2} \Psi_{\mu}(\tau, z)\right|_{z=0}
$$

The kernel $g_{C}\left(\left\{\gamma_{j}\right\},\left\{c_{j}^{\lambda}\right\}\right)$ and therefore $\Psi_{\mu}$ is unchanged under a symplectic transformation (3.7), such that $h_{\left\{P_{j}\right\}, \mu}^{T}$ is invariant under spectral flow as required. The number of terms in the sum over $\mu_{j}$ in (4.53) is given by $N_{q}$ (4.13).

To determine the modular properties of $\Psi_{\mu}$, we consider first the generating function of $f_{C}(2.45)$, which enumerates the number of scaling charge configurations for a given total charge. We define this function $\Phi_{\mu}$ as the following theta series,

$$
\Phi_{\mu}(\tau)=\sum_{Q \in \underline{\Lambda}_{\mu}^{*}} f_{C}\left(\left\{\gamma_{j}\right\},\left\{c_{j}^{\lambda}\right\}\right) q^{-Q^{2} / 2}
$$

with $f_{C}\left(\left\{\gamma_{j}\right\},\left\{c_{j}^{\lambda}\right\}\right)$ as in $(2.45)$.

The following subsections will demonstrate that $\Phi_{\mu}(\tau)$ is a convergent $q$-series, which can be completed to a function $\widehat{\Phi}_{\boldsymbol{\mu}}$ which transform as a vector-valued modular form. The transformation properties under the $S$ and $T$ transformations are

$$
\begin{aligned}
& \widehat{\Phi}_{\boldsymbol{\mu}}(-1 / \tau,-1 / \bar{\tau})=-\frac{(-i \tau)^{b_{2}}}{\sqrt{\mid \underline{\boldsymbol{\Lambda}}^{*} / \underline{\boldsymbol{\Lambda} \mid}}} e^{\pi i \boldsymbol{K}^{2} / 2} \sum_{\boldsymbol{\nu} \in \underline{\boldsymbol{\Lambda}}^{*} / \underline{\boldsymbol{\Lambda}}} e^{2 \pi i \mu . \nu} \widehat{\Phi}_{\boldsymbol{\nu}}(\tau, \bar{\tau}), \\
& \widehat{\Phi}_{\boldsymbol{\mu}}(\tau+1, \bar{\tau}+1)=e^{\pi i(\boldsymbol{\mu}+\boldsymbol{K} / 2)^{2}} \widehat{\Phi}_{\boldsymbol{\mu}}(\tau, \bar{\tau}) .
\end{aligned}
$$

The partition function $\widehat{\Psi}_{\mu}(\tau)$ can be obtained by introducing a suitable elliptic variable in $\widehat{\Phi}_{\mu}$ and subsequently differentiating twice to this variable. As a result, the modular transformations of the completed function $\widehat{\Phi}_{\mu}$ equal those of the completed $\widehat{\Psi}_{\mu}$ except that the weight of $\widehat{\Psi}_{\boldsymbol{\mu}}$ is increased by two compared to $\widehat{\Phi}_{\boldsymbol{\mu}}$. The weight of $\widehat{\Psi}_{\boldsymbol{\mu}}$ is thus $b_{2}+2$. The non-holomorphic terms are determined in this way in section 4.5, specifically eq. (4.116). The end result is that $\widehat{\Psi}_{\boldsymbol{\mu}}$ transforms as

$$
\begin{aligned}
& \widehat{\Psi}_{\boldsymbol{\mu}}(-1 / \tau,-1 / \bar{\tau})=\frac{(-i \tau)^{b_{2}+2}}{\sqrt{\left|\underline{\boldsymbol{\Lambda}}^{*} / \underline{\boldsymbol{\Lambda}}\right|}} e^{\pi i \boldsymbol{K}^{2} / 2} \sum_{\boldsymbol{\nu} \in \underline{\boldsymbol{\Lambda}}^{*} / \underline{\boldsymbol{\Lambda}}} e^{2 \pi i \boldsymbol{\mu} . \boldsymbol{\nu}} \widehat{\Psi}_{\boldsymbol{\nu}}(\tau, \bar{\tau}), \\
& \widehat{\Psi}_{\boldsymbol{\mu}}(\tau+1, \bar{\tau}+1)=e^{\pi i(\boldsymbol{\mu}+\boldsymbol{K} / 2)^{2}} \widehat{\Psi}_{\boldsymbol{\mu}}(\tau, \bar{\tau}) .
\end{aligned}
$$

Therefore, the completion of $h_{\left\{P_{j}\right\}, \mu}^{3 T}(4.53)$,

$$
\widehat{h}_{\left\{P_{j}\right\}, \mu}^{3 T}(\tau, \bar{\tau})=\sum_{\substack{\mu_{j} \in \Lambda_{j}^{*} / \Lambda_{j}, j=1,2,3, \mu_{1}+\mu_{2}+\mu_{3}=\mu}} \widehat{h}_{P_{1}, \mu_{1}}^{T}(\tau) \widehat{h}_{P_{2}, \mu_{2}}^{T}(\tau) \widehat{h}_{P_{3}, \mu_{3}}^{T}(\tau) \widehat{\Psi}_{\mu}(\tau),
$$

transforms as $\widehat{h}_{P, \mu}(3.21)$ as we aimed to show. We can furthermore combine $\widehat{h}_{P, \mu}$ with the theta series $\Theta_{\mu}$,

$$
\widehat{\mathcal{Z}}_{P}^{3 T}(\tau, C, t)=\sum_{\sum_{j=1}^{3} P_{j}=P} \sum_{\mu \in \Lambda^{*} / \Lambda} \widehat{h}_{\left\{P_{j}\right\}, \mu}^{3 T}(\tau, \bar{\tau}) \Theta_{\mu}(\tau, \bar{\tau}, C, B) .
$$


We can then decompose the attractor partition $\widehat{\mathcal{Z}}_{P}^{\lambda}$ in terms of the multi-core partition functions $\widehat{\mathcal{Z}}_{P}^{n T}$,

$$
\widehat{\mathcal{Z}}_{P}^{\lambda}(\tau, C, t)=\widehat{\mathcal{Z}}_{P}^{T}(\tau, C, t)+\widehat{\mathcal{Z}}_{P}^{3 T}(\tau, C, t)+\ldots
$$

Since the partition functions transform the same way, this raises the question which terms are captured by the MSW conformal field theory. As mentioned in the introduction, it will also be interesting to deduce the non-holomorphic terms of $\widehat{\mathcal{Z}}_{P}^{T}$ using those determined for $\widehat{\mathcal{Z}}_{P}^{3 T}$ in this paper, and those for $\widehat{\mathcal{Z}}_{P}^{\lambda}$ in [29].

\subsection{Convergence}

A crucial aspect of $\Phi_{\mu}$ (and $\Psi_{\mu}$ ) is whether the sum on the r.h.s. of (4.58) (and (4.54)) is convergent. If $\boldsymbol{Q}^{2}$ would be negative definite, convergence of these series would be guaranteed. However this is not the case since the electric charge lattice has signature $\left(2,2 b_{2}-2\right)$, i.e. has 2 positive directions.

To prove the convergence, we first introduce a theta series $\Theta_{\mu}[\mathcal{K}](\tau)$ with kernel $\mathcal{K}$ for a generic indefinite theta lattice $L$ and $\mu \in L^{*}$,

$$
\Theta_{\mu}[\mathcal{K}](\tau ; L)=\sum_{x \in L+\mu} \mathcal{K}(x) q^{-B(x) / 2},
$$

with integral quadratic form $B$. If $L$ is negative definite, we also use

$$
\theta_{\mu}(\tau ; L)=\Theta_{\mu}[1](\tau ; L)
$$

For an indefinite lattice the kernel $\mathcal{K}(x)=\mathcal{K}(x, \mathcal{V})$ depends on a collection

$$
\mathcal{V}=\left\{V_{1}, V_{2}, \ldots, V_{N}\right\}
$$

of positive vectors. For signature $\left(2,2 b_{2}-2\right), \mathcal{K}(x, \mathcal{V})$ can be expressed as $[28,36,75]$

$$
\mathcal{K}(x, \mathcal{V})=\frac{1}{4}\left(w(\mathcal{V})+\sum_{j=1}^{N} \operatorname{sgn}\left(B\left(x, V_{j}\right)\right) \operatorname{sgn}\left(B\left(x, V_{j+1}\right)\right)\right)
$$

where for any strictly positive vector $v \in L, v^{2}>0$,

$$
w(\mathcal{V})=-\sum_{j=1}^{N} \operatorname{sgn}\left(B\left(v, V_{j}\right)\right) \operatorname{sgn}\left(B\left(v, V_{j+1}\right)\right),
$$

which is independent of the choice of positive vector $v$ [75]. There are various sufficient conditions for convergence put forward in the literature [28, 36, 38, 39, 75]. We will consider here the following $N$-gon conditions put forward in [28, 36, 75], which read

$$
\begin{aligned}
B\left(V_{j}, V_{j}\right) & >0, \\
B\left(V_{j}, V_{j}\right) B\left(V_{j+1}, V_{j+1}\right)-B\left(V_{j}, V_{j+1}\right)^{2} & >0, \\
B\left(V_{j}, V_{j}\right) B\left(V_{j-1}, V_{j+1}\right)-B\left(V_{j}, V_{j-1}\right) B\left(V_{j}, V_{j+1}\right) & <0 .
\end{aligned}
$$


Now let us return to the sum (4.58) at hand. It comprises of six individual sums, which are each of the form

$$
\sum_{\substack{Q_{i} \in \mu_{i}+\Lambda_{i}+P_{i} / 2 \\ Q_{1}+Q_{2}+Q_{3}=\mu+P / 2}} F_{\ell}(a, b, c)(-1)^{a+b+c} q^{Q^{2} / 2-\sum_{i}\left(Q_{i}\right)_{i}^{2} / 2}=\sum_{\boldsymbol{Q} \in \underline{\Lambda}_{\mu}^{*}} F_{\ell}(a, b, c)(-1)^{a+b+c} q^{-Q^{2} / 2},
$$

with $\ell=1, \ldots, 6$.

The simplification $F_{\text {total }}(a, b, c)$ for $\sum_{j=1}^{6} F_{j}(a, b, c)$ put forward in (2.44) is precisely of the form (4.66) with $N=3$. To present the vectors, we define

$$
\begin{aligned}
& C_{a}=\left(-P_{2}, P_{1}, 0\right), \\
& C_{b}=\left(0,-P_{3}, P_{2}\right), \\
& C_{c}=\left(P_{3}, 0,-P_{1}\right),
\end{aligned}
$$

such that $C_{a} \cdot \boldsymbol{Q}=a, C_{b} \cdot \boldsymbol{Q}=b$ and $C_{c} \cdot \boldsymbol{Q}=c$, with $a, b$ and $c$ as in (4.2). The $V_{j}$ are then identified with $C_{j} \in \underline{\boldsymbol{\Lambda}}$ with the $C_{j}$ given by,

$$
\begin{aligned}
& C_{1}=C_{a}+C_{b}-C_{c}=\left(-P_{2}-P_{3}, P_{1}-P_{3}, P_{1}+P_{2}\right), \\
& C_{2}=C_{a}-C_{b}+C_{c}=\left(-P_{2}+P_{3}, P_{1}+P_{3},-P_{1}-P_{2}\right), \\
& C_{3}=-C_{a}+C_{b}+C_{c}=\left(P_{2}+P_{3},-P_{1}-P_{3},-P_{1}+P_{2}\right) .
\end{aligned}
$$

If we assume that $P_{j}$ is an ample divisor for each $j \in 1,2,3$, triple intersections $P_{i} P_{j} P_{k}>0$ for all $i, j, k \in\{1,2,3\}$. The conditions for convergence (4.68) are then satisfied.

It is also useful to consider the convergence for the kernel due to a single permutation separately, since the different permuations are weighted by a different factor in $g_{C}$. The vectors $V_{j}$ for the kernel $F^{*}(123)(2.40)$ can be chosen as $C_{j}^{(123)}$,

$$
\begin{aligned}
& C_{1}^{(123)}=C_{a}-C_{c}=\left(C_{1}-C_{2}\right) / 2, \\
& C_{2}^{(123)}=C_{b}-C_{c}=\left(C_{1}-C_{3}\right) / 2, \\
& C_{3}^{(123)}=C_{c}-C_{a}-C_{b}=-C_{1} .
\end{aligned}
$$

Again one may verify that with the assumption $P_{i} P_{j} P_{k}>0$ for all $i, j, k \in\{1,2,3\}$, these vectors satisfy the conditions for convergence (4.68).

\subsection{Modular completion of $\Phi_{\mu}$}

Having discussed the convergence of $\Phi_{\boldsymbol{\mu}}$, we proceed in this section to discuss its modularity. Since $\Phi_{\mu}$ is a sum over a subset of an indefinite lattice, the function is not modular in the classical sense. Our task is to determine a modular completion $\widehat{\Phi}_{\boldsymbol{\mu}}$, which differes from $\Phi_{\mu}$ by subleading non-holomorphic terms, and which does transform as a modular form. Essentially, products of sgn-functions are replaced by a generalized error function [36]. Following this approach, we will demonstrate that the difference between $\widehat{\Phi}_{\boldsymbol{\mu}}$ and $\Phi_{\boldsymbol{\mu}}$, is given by iterated integrals of modular forms. 
Such non-holomorphic contributions have appeared in similar contexts. In specific cases, the non-holomorphic contributions are derived from different physical points of view, for example the continuum of multi-particle states in $\mathbb{R}^{4}[58]$, or the quantum field theory on the world volume of the D-branes [44, 47, 48], or the perspective of D3-instantons in the hypermultiplet moduli space [18].

To this end let us consider $F_{\text {total }}(a, b, c)$ in (2.44). Under modular completion, one adds certain extra terms to $\operatorname{sgn}\left(V_{1}, x\right) \operatorname{sgn}\left(V_{2}, x\right)$, therefore replacing $\operatorname{sgn}\left(V_{1}, x\right) \operatorname{sgn}\left(V_{2}, x\right)$ with the double error function $E_{2}\left(\alpha, u_{1}, u_{2}\right)$ :

$$
\operatorname{sgn}\left(V_{1}, x\right) \operatorname{sgn}\left(V_{2}, x\right) \rightarrow E_{2}\left(\alpha, \sqrt{2 \tau_{2}} \boldsymbol{u}\right)
$$

with [36]

$$
E_{2}(\alpha ; \boldsymbol{u})=\int_{\mathbb{R}^{2}} e^{-\pi\left(u_{1}-u_{1}^{\prime}\right)^{2}-\pi\left(u_{2}-u_{2}^{\prime}\right)^{2}} \operatorname{sgn}\left(u_{2}^{\prime}\right) \operatorname{sgn}\left(u_{1}^{\prime}+\alpha u_{2}^{\prime}\right) d u_{1}^{\prime} d u_{2}^{\prime},
$$

whose arguments are given in terms of $V_{1}, V_{2}$ and $x$ by

$$
\begin{aligned}
& \alpha=\alpha\left(V_{1}, V_{2}\right)=\frac{\left(V_{1}, V_{2}\right)}{\sqrt{V_{1}^{2} V_{2}^{2}-\left(V_{1}, V_{2}\right)^{2}}}, \\
& \boldsymbol{u}=\boldsymbol{u}\left(V_{1}, V_{2} ; x\right)=\left(u_{1}\left(V_{1}, V_{2} ; x\right), u_{2}\left(V_{1}, V_{2} ; x\right)\right),
\end{aligned}
$$

with

$$
\begin{aligned}
& u_{1}\left(V_{1}, V_{2} ; x\right)=\frac{\left(V_{1 \perp 2}, x\right)}{\sqrt{\left(V_{1 \perp 2}, V_{1 \perp 2}\right)}}, \\
& u_{2}\left(V_{1}, V_{2} ; x\right)=\frac{\left(V_{2}, x\right)}{\sqrt{\left(V_{2}, V_{2}\right)}}
\end{aligned}
$$

and $V_{1 \perp 2}$ the component of $V_{1}$ orthogonal to $V_{2}$,

$$
V_{1 \perp 2}=V_{1}-\frac{\left(V_{1}, V_{2}\right)}{\left(V_{2}, V_{2}\right)} V_{2}
$$

To stress the dependance of $E_{2}$ on the vectors $V_{1}, V_{2}$ and $x$, we will also use $E_{2}$ with alternative arguments,

$$
E_{2}(\alpha, \boldsymbol{u}) \equiv E_{2}\left(V_{1}, V_{2} ; x\right)
$$

with the identifications as in (4.75).

In addition to (4.74), another (equivalent) expression for $E_{2}$ is in terms of Eichler integrals. To this end, we first define the Eichler integrals $M_{1}$,

$$
M_{1}(u)=\left\{\begin{array}{cc}
\frac{i u}{\sqrt{2 \tau_{2}}} q^{\frac{u^{2}}{4 \tau_{2}}} \int_{-\bar{\tau}}^{i \infty} \frac{e^{\frac{i \pi u^{2} w}{2 \tau_{2}}}}{\sqrt{-i(w+\tau)}} d w, & u \neq 0 \\
0, & u=0 .
\end{array}\right.
$$


and the (iterated) Eichler integral $M_{2}$ and $m_{2}$, for $u_{1} \neq 0$, and $u_{2}-\alpha u_{1} \neq 0$,

$$
\begin{aligned}
& m_{2}\left(u_{1}, u_{2}\right)=\left\{\begin{array}{cl}
\frac{u_{1} u_{2}}{2 \tau_{2}} q^{\frac{u_{1}^{2}}{4 \tau_{2}}+\frac{u_{2}^{2}}{4 \tau_{2}}} \int_{-\bar{\tau}}^{i \infty} d w_{2} \int_{w_{2}}^{i \infty} d w_{1} \frac{e^{\frac{\pi i u_{1}^{2} w_{1}}{2 \tau_{2}}+\frac{\pi i u_{2}^{2} w_{2}}{2 \tau_{2}}}}{\sqrt{-\left(w_{1}+\tau\right)\left(w_{2}+\tau\right)}}, & u_{1} \neq 0 \\
0, & u_{1}=0
\end{array}\right. \\
& M_{2}\left(\alpha ; u_{1}, u_{2}\right)= \begin{cases}-m_{2}\left(u_{1}, u_{2}\right)-m_{2}\left(\frac{u_{2}-\alpha u_{1}}{\sqrt{1+\alpha^{2}}}, \frac{u_{1}+\alpha u_{2}}{\sqrt{1+\alpha^{2}}}\right) & u_{1} \neq 0, u_{2}-\alpha u_{1} \neq 0, \\
-m_{2}\left(\frac{u_{2}-\alpha u_{1}}{\sqrt{1+\alpha^{2}}}, \frac{u_{1}+\alpha u_{2}}{\sqrt{1+\alpha^{2}}}\right) & u_{1}=0, u_{2} \neq 0, \\
-m_{2}\left(u_{1}, u_{2}\right) & u_{1} \neq 0, u_{2}-\alpha u_{1}=0, \\
\frac{2}{\pi} \arctan \alpha & u_{1}=u_{2}=0 .\end{cases}
\end{aligned}
$$

With $\boldsymbol{u}=\left(u_{1}, u_{2}\right)$ as before, the double error function $E_{2}$ is then defined as a linear combination of $M_{1}$ and $M_{2}[36,57]$

$$
\begin{aligned}
E_{2}(\alpha ; \boldsymbol{u})= & \operatorname{sgn}\left(u_{2}\right) \operatorname{sgn}\left(u_{1}+\alpha u_{2}\right)+\operatorname{sgn}\left(u_{1}\right) M_{1}\left(u_{2}\right) \\
& +\operatorname{sgn}\left(u_{2}-\alpha u_{1}\right) M_{1}\left(\frac{u_{1}+\alpha u_{2}}{\sqrt{1+\alpha^{2}}}\right)+M_{2}\left(\alpha ; u_{1}, u_{2}\right) .
\end{aligned}
$$

See [36] for other representations of $E_{2}$.

Thus $E_{2}$ consists of the original $\operatorname{sgn}\left(V_{1}, x\right) \operatorname{sgn}\left(V_{2}, x\right)$ plus four more terms. Noting that

$$
\begin{aligned}
& \frac{u_{2}\left(V_{2}, x\right)-\alpha u_{1}\left(V_{1}, V_{2}, x\right)}{\sqrt{1+\alpha^{2}}}=u_{1}\left(V_{2}, V_{1}, x\right), \\
& \frac{u_{1}\left(V_{1}, V_{2}, x\right)+\alpha u_{2}\left(V_{2}, x\right)}{\sqrt{1+\alpha^{2}}}=u_{2}\left(V_{1}, x\right),
\end{aligned}
$$

we can write

$$
\begin{aligned}
E_{2}(\alpha ; \boldsymbol{u})= & \operatorname{sgn}\left(V_{1} \cdot x\right) \operatorname{sgn}\left(V_{2} \cdot x\right)+\operatorname{sgn}\left(u_{1}\left(V_{1}, V_{2}, x\right)\right) M_{1}\left(u_{2}\left(V_{2}, x\right)\right) \\
& +\operatorname{sgn}\left(u_{1}\left(V_{2}, V_{1}, x\right)\right) M_{1}\left(u_{2}\left(V_{1}, x\right)\right) \\
& -m_{2}\left(u_{1}\left(V_{1}, V_{2}, x\right), u_{2}\left(V_{2}, x\right)\right)-m_{2}\left(u_{1}\left(V_{2}, V_{1}, x\right), u_{2}\left(V_{1}, x\right)\right) .
\end{aligned}
$$

$E_{2}$ satisfies an identity similar to $(2.39)$. This reads

$$
E_{2}\left(V_{1}, V_{1}+V_{2} ; x\right)+E_{2}\left(V_{2}, V_{1}+V_{2} ; x\right)-E_{2}\left(V_{1}, V_{2} ; x\right)=1
$$

and is valid for any choice of the arguments such that the corresponding $\alpha, u_{1}$ and $u_{2}$ 's are in $\mathbb{R}$.

The sum at hand (2.44) has the form

$$
\operatorname{sgn}\left(C_{1} \cdot x\right) \operatorname{sgn}\left(C_{2} \cdot x\right)+\operatorname{sgn}\left(C_{2} \cdot x\right) \operatorname{sgn}\left(C_{3} \cdot x\right)+\operatorname{sgn}\left(C_{3} \cdot x\right) \operatorname{sgn}\left(C_{1} \cdot x\right),
$$

with $C_{1}, C_{2}, C_{3}$ given in (4.71). The modular completion $\widehat{\Phi}_{\boldsymbol{\mu}}$ of $\Phi_{\boldsymbol{\mu}}$ (4.58) follows by the adding to the coefficient $f_{C}(2.45)$ the following terms

$$
\frac{1}{4} \sum_{\ell=1,2,3}\left[E_{2}\left(C_{\ell}, C_{\ell+1} ; \sqrt{2 \tau_{2}} x\right)-\operatorname{sgn}\left(C_{\ell} \cdot x\right) \operatorname{sgn}\left(C_{\ell+1} \cdot x\right)-A_{\ell} \delta_{\left(C_{\ell} \cdot x\right)} \delta_{\left(C_{\ell+1} \cdot x\right)}\right] .
$$


This essentially amounts to replacing $f_{C}$ by a linear combination of $E_{2}$ 's. Since the latter satisfies Vignéras equation, modular transformation properties are ensured [55].

Our first aim is to determine the value of $A_{\ell}$ such that the completion is subleading, i.e. it vanishes in the limit that $\operatorname{Im}(\tau)=\tau_{2} \rightarrow \infty$. This follows from realizing that the difference $E_{2}\left(C_{\ell}, C_{\ell+1} ; \sqrt{2 \tau_{2}} x\right)-\operatorname{sgn}\left(C_{\ell} \cdot x\right) \operatorname{sgn}\left(C_{\ell+1} . x\right)$ vanishes in this limit except if $\left(C_{\ell} \cdot x\right)=\left(C_{\ell+1} \cdot x\right)=0$, when it equals $\frac{2}{\pi} \arctan \left(\alpha_{\ell}\right), \alpha_{\ell}=\alpha\left(C_{\ell}, C_{\ell+1}\right)$ (4.75). Requiring that (4.87) vanish, we thus arrive at

$$
A_{\ell}=\frac{2}{\pi} \arctan \left(\alpha_{\ell}\right)
$$

Surprisingly this implies that $A_{\ell}$ can be irrational, as we will see in the explicit case studies in section 6 . This of course obstructs an interpretation as a "counting" function for the coefficients to which $A_{\ell}$ contribute. On the other hand, since the function $\Phi_{\mu}$ is not a proper physical partition function summing over a Hilbert space, we are not very concerned about this.

To proceed with determining the completion, we rearrange the terms and write (4.87) as

$$
\begin{aligned}
& \frac{1}{4} \sum_{\ell=1,2,3}\left[\operatorname{sgn}\left(u_{1}\left(C_{\ell+1}, C_{\ell}, x\right)\right)+\operatorname{sgn}\left(u_{1}\left(C_{\ell-1}, C_{\ell}, x\right)\right)\right] M_{1}\left(\sqrt{2 \tau_{2}} u_{2}\left(C_{\ell}, x\right)\right) \\
& -m_{2}\left(\sqrt{2 \tau_{2}} u_{1}\left(C_{\ell+1}, C_{\ell}, x\right), \sqrt{2 \tau_{2}} u_{2}\left(C_{\ell}, x\right)\right)-m_{2}\left(\sqrt{2 \tau_{2}} u_{1}\left(C_{\ell-1}, C_{\ell}, x\right), \sqrt{2 \tau_{2}} u_{2}\left(C_{\ell}, x\right)\right) .
\end{aligned}
$$

We determine now the $A_{\ell}$ by requiring that this expression vanishes in the limit $y \rightarrow \infty$.

Using these expressions, we thus naturally separate the holomorphic part $\Phi_{\boldsymbol{\mu}}$ from the completion $\widehat{\Phi}_{\mu}$,

$$
\widehat{\Phi}_{\boldsymbol{\mu}}(\tau, \bar{\tau})=\Phi_{\boldsymbol{\mu}}(\tau)+R_{\boldsymbol{\mu}}^{\Phi}(\tau, \bar{\tau}),
$$

with the non-holomorphic completion $R_{\mu}^{\Phi}$ defined by

$$
\begin{aligned}
R_{\mu}^{\Phi}(\tau, \bar{\tau})= & \sum_{\boldsymbol{Q} \in \underline{\boldsymbol{\Lambda}}_{\mu}^{*}} \sum_{\ell=1,2,3} \\
& {\left[\left[\operatorname{sgn}\left(u_{1}\left(C_{\ell+1}, C_{\ell}, x\right)\right)+\operatorname{sgn}\left(u_{1}\left(C_{\ell-1}, C_{\ell}, x\right)\right)\right] M_{1}\left(\sqrt{2 \tau_{2}} u_{2}\left(C_{\ell}, x\right)\right)\right.} \\
& -m_{2}\left(\sqrt{2 \tau_{2}} u_{1}\left(C_{\ell+1}, C_{\ell}, x\right), \sqrt{2 \tau_{2}} u_{2}\left(C_{\ell}, x\right)\right) \\
& \left.-m_{2}\left(\sqrt{2 \tau_{2}} u_{1}\left(C_{\ell-1}, C_{\ell}, x\right), \sqrt{2 \tau_{2}} u_{2}\left(C_{\ell}, x\right)\right)\right] \\
& \times(-1)^{\boldsymbol{K} \cdot \boldsymbol{Q}} q^{-Q^{2} / 2}
\end{aligned}
$$

Our next aim is to write the non-holomorphic part as an (iterated) integral over modular forms. This makes the modular properties of the holomorphic $q$-series manifest, since the modular properties of integrals of modular forms are readily determined. Moreover, it is straightforward to determine the non-holomorphic anomaly.

To determine this form of the non-holomorphic part, we write $R_{\mu}^{\Phi}$ as

$$
R_{\boldsymbol{\mu}}^{\Phi}(\tau, \bar{\tau})=\sum_{\ell=1,2,3}\left[R_{\boldsymbol{\mu}, 1, \ell}(\tau, \bar{\tau})+R_{\boldsymbol{\mu}, 2,(\ell-1, \ell)}(\tau, \bar{\tau})+R_{\boldsymbol{\mu}, 2,(\ell+1, \ell)}(\tau, \bar{\tau})\right]
$$




\begin{tabular}{|c|c|c|c|}
\hline term & form & dual lattice & signature \\
\hline $\boldsymbol{Q}^{2}$ & $\underline{D}$ & $\underline{\boldsymbol{\Lambda}}_{\boldsymbol{\mu}}^{*}$ & $\left(2,2 b_{2}-2\right)$ \\
\hline$u_{2, \ell}^{2}$ & $\underline{D}_{\ell}=\left|C_{\ell}\right|^{2}$ & $\left(L_{\ell}\right)_{\boldsymbol{\mu}}^{*} \subset \underline{\boldsymbol{\Lambda}}_{\boldsymbol{\mu}}^{*}$ & $(1,0)$ \\
\hline $\boldsymbol{Q}^{2}-u_{2, \ell}^{2}$ & $\underline{D}_{\perp \ell}$ & $\left(L_{\ell}^{\perp}\right)_{\boldsymbol{\mu}}^{*} \subset \underline{\boldsymbol{\Lambda}}_{\boldsymbol{\mu}}^{*}$ & $\left(1,2 b_{2}-2\right)$ \\
\hline$u_{1,(k, l)}^{2}$ & $\underline{D}_{(k, l)}=\left|C_{k \perp \ell}\right|^{2}$ & $\left(L_{k \ell}\right)_{\boldsymbol{\mu}}^{*} \subset\left(L_{\ell}^{\perp}\right)_{\boldsymbol{\mu}}^{*}$ & $(1,0)$ \\
\hline $\boldsymbol{Q}^{2}-u_{1,(k, l)}^{2}-u_{2, \ell}^{2}$ & $\underline{D}_{(k \ell)}^{\perp}$ & $\left(L_{k \ell}^{\perp}\right)_{\boldsymbol{\mu}}^{*} \subset\left(L_{\ell}^{\perp}\right)_{\boldsymbol{\mu}}^{*}$ & $\left(0,2 b_{2}-2\right)$ \\
\hline
\end{tabular}

Table 1. Lattices and quadratic forms associated to the splitting in equation (4.94).

and $R_{\boldsymbol{\mu}, 1, \ell}, R_{\boldsymbol{\mu}, 2,(k, \ell)}$ are defined as

$$
\begin{aligned}
R_{\boldsymbol{\mu}, 1, \ell}(\tau, \bar{\tau}) & =\sum_{\boldsymbol{Q} \in \underline{\boldsymbol{\Lambda}}_{\mu}^{*}}\left[\operatorname{sgn}\left(u_{1,(\ell+1, \ell)}+\operatorname{sgn}\left(u_{1,(\ell-1, \ell)}\right)\right] M_{1}\left(\sqrt{2 y} u_{2, \ell}\right)(-1)^{\boldsymbol{K} \cdot \boldsymbol{Q}} q^{-\boldsymbol{Q}^{2} / 2},\right. \\
R_{\boldsymbol{\mu}, 2,(k, \ell)}(\tau, \bar{\tau}) & =-\sum_{\boldsymbol{Q} \in \underline{\boldsymbol{\Lambda}}_{\mu}^{*}} m_{2}\left(\sqrt{2 \tau_{2}} u_{1,(k, l)}, \sqrt{2 \tau_{2}} u_{2, \ell}\right)(-1)^{\boldsymbol{K} \cdot \boldsymbol{Q}} q^{-\boldsymbol{Q}^{2} / 2}
\end{aligned}
$$

where we have used the abbreviations $u_{1,(k, \ell)}=u_{1}\left(C_{k}, C_{\ell}, x\right), u_{2, \ell}=u_{2}\left(C_{\ell}, x\right)$.

To evaluate the sums (4.93), it is useful to split $\boldsymbol{Q}^{2}$ as follows

$$
\boldsymbol{Q}^{2}=u_{1,(k, \ell)}^{2}+u_{2, \ell}^{2}+\left(\boldsymbol{Q}^{2}-u_{1,(k, \ell)}^{2}-u_{2, \ell}^{2}\right),
$$

$u_{1,(k, \ell)}^{2}, u_{2, \ell}^{2}$ and $\left(\boldsymbol{Q}^{2}-u_{1,(k, \ell)}^{2}-u_{2, \ell}^{2}\right)$ are naturally associated with quadratic forms and lattices as described in table 1 .

We let $L_{\ell}$ be the 1-dimensional lattice spanned by $C_{\ell}$. The quadratic form is a number in this case, $D_{\ell}=\left|C_{\ell}\right|^{2}$. We denote the dual lattice with quadratic form $\left|C_{\ell}\right|^{-2}$ by $\left(L_{\ell}\right)^{*}$. The projection of $\boldsymbol{\mu}$ to $\left(L_{\ell}\right)^{*}$ is $\mu_{\ell}=\left(\boldsymbol{\mu} . C_{\ell}\right) C_{\ell} \in \mathbb{Z}^{2 b_{2}}$. To express a generic vector in $\underline{\Lambda}_{\boldsymbol{\mu}}^{*}$ as an element of $\left(L_{\ell}\right)^{*} \oplus\left(L_{\ell}^{\perp}\right)^{*}$, we introduce glue vectors $\rho$. We denote by $\left(L_{\ell}\right)_{\mu}^{*}$ the set of vectors $\mu_{\ell} \bmod L_{\ell} \in\left(L_{\ell}\right)^{*}$, and by $\left(L_{\ell}\right)_{\mu+\rho}^{*}$ the set of vectors $\mu_{\ell}+\rho \bmod L_{\ell} \in\left(L_{\ell}\right)^{*}$. We introduce similar notation for $L_{\ell}^{\perp}$. Using this notation, the direct $\operatorname{sum}\left(L_{\ell}\right)_{\mu}^{*} \oplus\left(L_{\ell}^{\perp}\right)_{\mu}^{*}$ is a subset of the lattice $\underline{\boldsymbol{\Lambda}}_{\boldsymbol{\mu}}^{*},\left(L_{\ell}\right)_{\boldsymbol{\mu}}^{*} \oplus\left(L_{\ell}^{\perp}\right)_{\boldsymbol{\mu}}^{*} \subset \underline{\Lambda}_{\boldsymbol{\mu}}^{*}$.

A generic element $\boldsymbol{k}$ of $\underline{\boldsymbol{\Lambda}}_{\boldsymbol{\mu}}^{*}$ can be written as a sum, $\boldsymbol{k}=\boldsymbol{l}_{\ell}+\boldsymbol{l}_{\ell}^{\perp} \in\left(L_{\ell}\right)_{\boldsymbol{\mu}, \rho}^{*} \oplus\left(L_{\ell}^{\perp}\right)_{\boldsymbol{\mu}, \rho}^{*}$ for some $\rho$, where the projection $\boldsymbol{l}_{\ell}$ to $\left(L_{\ell}^{\perp}\right)^{*}$ vanishes, and similarly for the projection of $\boldsymbol{l}_{\ell}^{\perp}$ to $\left(L_{\ell}\right)^{*}$. We have a similar decomposition of vectors in $\left(L_{\ell}\right)^{\perp}$ with respect to the decomposition $\left(L_{k \ell}\right)^{*} \oplus\left(L_{k \ell}^{\perp}\right)^{*}$. The representatives of minimal length in $\left(L_{\ell}\right)^{*} \oplus\left(L_{\ell}^{\perp}\right)^{*}$ appearing in such splits are called "glue vectors". We use glue vectors $\rho$ for the splitting $\left(L_{\ell}\right)_{\boldsymbol{\mu}, \rho}^{*} \oplus\left(L_{\ell}^{\perp}\right)_{\boldsymbol{\mu}, \rho}^{*}$ and $\nu$ for the splitting $\left(L_{k \ell}\right)^{*} \oplus\left(L_{k \ell}^{\perp}\right)^{*}$. We then have,

$$
\begin{aligned}
\underline{\Lambda}_{\mu}^{*} & =\sum_{\rho}\left(L_{\ell}\right)_{\mu+\rho}^{*} \oplus\left(L_{\ell}^{\perp}\right)_{\mu+\rho}^{*}, \\
\left(L_{\ell}^{\perp}\right)_{\rho}^{*} & =\sum_{\nu} \subset\left(L_{k}\right)_{\rho+\nu}^{*} \oplus\left(L_{k \ell}^{\perp}\right)_{\rho+\nu}^{*} .
\end{aligned}
$$

Now let us evaluate the sum $R_{\boldsymbol{\mu}, 1}$ and $R_{\boldsymbol{\mu}, 2}$ (4.93). The embedding of $L_{\ell}$ in $\underline{\boldsymbol{\Lambda}}_{\boldsymbol{\mu}} \otimes \mathbb{Q}$ is spanned by $k C_{\ell}$ with $k \in \mathbb{Z}+\rho$ where $\rho \in \mathbb{Q}$ is (the projection of) the glue vector. Then, 
$u_{2, \ell}=\left|C_{\ell}\right| k$, such that $R_{\mu, 1, \ell}$ reads

$$
\begin{aligned}
R_{\mu, 1, \ell}(\tau, \bar{\tau})= & \sum_{\rho} \sum_{\substack{l_{\ell}^{\perp} \in\left(L_{\ell}^{\perp}\right)^{*} \\
k \in \mathbb{Z}+\rho+\rho}}\left(\operatorname{sgn}\left(u_{1,(\ell-1, \ell)}\right)+\operatorname{sgn}\left(u_{1,(\ell+1, \ell)}\right)\right) \\
& \times(-1)^{K . l_{\ell}^{\perp}+K \cdot C_{\ell} k} M_{1}\left(\sqrt{2 \tau_{2}}\left|C_{\ell}\right| k\right) q^{-\left(l_{\ell}^{\perp}\right)^{2} / 2-C_{\ell}^{2} k^{2} / 2} .
\end{aligned}
$$

To write this more compactly, we define for a generic lattice $L$ of signature $(1, \operatorname{dim}(L)-1)$ and characteristic vector $K$,

$$
\Theta_{\alpha}\left(\tau ; L,\left\{V, V^{\prime}\right\}\right):=\sum_{x \in L+\alpha}\left(\operatorname{sgn}(V, x)-\operatorname{sgn}\left(V^{\prime}, x\right)\right)(-1)^{K \cdot x} q^{-x^{2} / 2} .
$$

Convergence of $\Theta_{\alpha}$ requires [34]

$$
V^{2}>0,\left(V^{\prime}\right)^{2}>0,\left(V, V^{\prime}\right)>0 .
$$

Moreover, we define the unary theta series $\Upsilon_{\alpha}$,

$$
\Upsilon_{\alpha}(\tau, M, N)=\sum_{x \in \mathbb{Z}+\alpha} x(-1)^{N x} e^{\pi i \tau M x^{2}} .
$$

For $\sigma \in \overline{\mathbb{H}}$, we introduce the period integral

$$
\begin{aligned}
R_{\alpha}(\tau, \sigma ; M, N) & :=i \sum_{x \in \mathbb{Z}+\alpha}(-1)^{N x} x \int_{-\sigma}^{i \infty} d w \frac{e^{\pi i w M x^{2}}}{\sqrt{-i(w+\tau)}} \\
& =i \int_{-\sigma}^{i \infty} d w \frac{\Upsilon_{\alpha}(w, M, N)}{\sqrt{-i(w+\tau)}} .
\end{aligned}
$$

The non-holomorphic modular completion $\widehat{\Theta}_{\alpha}$ of $\Theta_{\alpha}$ is expressed in terms of $R_{\alpha}$ as

$$
\begin{aligned}
\widehat{\Theta}_{\alpha}\left(\tau, \bar{\tau} ; L,\left\{V, V^{\prime}\right\}\right)= & \left.\Theta_{\alpha}\left(\tau ; L, V, V^{\prime}\right\}\right) \\
& +\sum_{\nu} \theta_{\alpha+\nu}\left(\tau ; L_{V}^{\perp}\right) R_{\alpha+\nu}\left(\tau, \bar{\tau} ; V^{2}, K_{L} \cdot V\right) \\
& -\sum_{\nu^{\prime}} \theta_{\alpha+\nu^{\prime}}\left(\tau ; L_{V^{\prime}}^{\perp}\right) R_{\alpha+\nu^{\prime}}\left(\tau, \bar{\tau} ;\left(V^{\prime}\right)^{2}, K_{L} \cdot V^{\prime}\right),
\end{aligned}
$$

with $\theta_{\alpha}$ as in (4.65).

Returning to $R_{\boldsymbol{\mu}, 1, \ell}$, we can now write this as

$$
R_{\boldsymbol{\mu}, 1, \ell}(\tau, \bar{\tau})=\sum_{\rho} \Theta_{\boldsymbol{\mu}+\rho}\left(\tau, L_{\ell}^{\perp},\left\{C_{\ell-1}, C_{\ell+1}\right\}\right) R_{\boldsymbol{\mu}+\rho}\left(\tau, \bar{\tau} ; C_{\ell}^{2}, \boldsymbol{K} . C_{\ell}\right) .
$$

The relations for convergence of $\Theta_{\boldsymbol{\mu}+\rho}$ are indeed satisfied for the vectors given in (4.71). We will continue to demonstrate that these are captured by the term $R_{\boldsymbol{\mu}, 2}$ in (4.92). We have

$$
\begin{aligned}
R_{\boldsymbol{\mu}, 2,(k, \ell)}(\tau, \bar{\tau})= & -\sum_{\boldsymbol{Q} \in \underline{\Lambda}_{\boldsymbol{\mu}}^{*}} m_{2}\left(\sqrt{2 \tau_{2}} u_{1}\left(C_{k}, C_{\ell}, \boldsymbol{Q}\right), \sqrt{2 \tau_{2}} u_{2}\left(C_{\ell}, \boldsymbol{Q}\right)\right)(-1)^{\boldsymbol{K} \cdot \boldsymbol{Q}} q^{-\boldsymbol{Q}^{2} / 2} \\
= & -\sum_{\boldsymbol{Q} \in \underline{\Lambda}_{\boldsymbol{\mu}}^{*}} u_{1}\left(C_{k}, C_{\ell}, \boldsymbol{Q}\right) u_{2}\left(C_{\ell}, \boldsymbol{Q}\right)(-1)^{\boldsymbol{K} \cdot \boldsymbol{Q}} q^{\left(u_{1}\left(C_{k}, C_{\ell}, \boldsymbol{Q}\right)^{2}+u_{2}\left(C_{\ell}, \boldsymbol{Q}\right)^{2}-\boldsymbol{Q}^{2}\right) / 2} \\
& \times \int_{-\bar{\tau}}^{i \infty} d w_{2} \int_{w_{2}}^{i \infty} d w_{1} \frac{e^{\pi i\left(w_{1} u_{1}\left(C_{k}, C_{\ell}, \boldsymbol{Q}\right)^{2}+w_{2} u_{2}\left(C_{\ell}, \boldsymbol{Q}\right)^{2}\right)}}{\sqrt{-\left(w_{1}+\tau\right)\left(w_{2}+\tau\right)}} .
\end{aligned}
$$


Using the splits of the lattices and $\Upsilon_{\alpha}$ (4.99), we can express this as

$$
\begin{aligned}
R_{\boldsymbol{\mu}, 2,(k, \ell)}(\tau, \bar{\tau})= & -\sum_{\rho} \theta_{\boldsymbol{\mu}+\rho+\nu}\left(\tau ; L_{k \ell}^{\perp}\right) \\
& \times \int_{-\bar{\tau}}^{i \infty} d w_{2} \int_{w_{2}}^{i \infty} d w_{1} \frac{\Upsilon_{\boldsymbol{\mu}+\rho}\left(w_{2} ; C_{\ell}^{2}, \boldsymbol{K} \cdot C_{\ell}\right) \Upsilon_{\boldsymbol{\mu}+\rho+\nu}\left(w_{1} ; C_{k \perp \ell}^{2}, \boldsymbol{K} \cdot C_{k \perp \ell}\right)}{\sqrt{-\left(w_{1}+\tau\right)\left(w_{2}+\tau\right)}} .
\end{aligned}
$$

Next, we aim to combine the sums in (4.91). We recognize the two $\Upsilon$ 's in the integrand in (4.104). The first, $\Upsilon_{\boldsymbol{\mu}+\rho}$, appears in the integrand of $R_{\boldsymbol{\mu}+\rho}$ on the r.h.s. of (4.102), whereas the second $\Upsilon_{\mu+\rho+\nu}$ matches with one of the non-holomorphic terms on the r.h.s. of (4.101). More precisely and concisely, we arrive at

$$
R_{\mu}^{\Phi}(\tau, \bar{\tau})=\sum_{\ell=1,2,3} i \int_{-\bar{\tau}}^{i \infty} d w \frac{\sum_{\rho} \widehat{\Theta}_{\boldsymbol{\mu}+\rho}\left(\tau,-w ; L_{\ell}^{\perp},\left\{C_{\ell-1} C_{\ell+1}\right\}\right) \Upsilon_{\boldsymbol{\mu}+\rho}\left(w ; C_{\ell}^{2}, \boldsymbol{K} \cdot C_{\ell}\right)}{\sqrt{-i(w+\tau)}}
$$

We thus have succeeded to express $R_{\mu}^{\Phi}$ in (4.90) as an iterated integral of modular forms.

Note that for $\ell=1$, there is a symmetry exchanging $P_{1} \leftrightarrow P_{2}$, and there are similar symmetries for $\ell=2$ and $\ell=3$. The form (4.105) makes the determination of the anti-holomorphic derivative of $\widehat{\Phi}_{\boldsymbol{\mu}}$ immediate. We have,

$$
\frac{\partial \widehat{\Phi}_{\mu}(\tau, \bar{\tau})}{\partial \bar{\tau}}=\sum_{\ell=1,2,3} \sum_{\rho} \frac{\widehat{\Theta}_{\mu+\rho}\left(\tau, \bar{\tau} ; L_{\ell}^{\perp},\left\{C_{\ell-1}, C_{\ell+1}\right\}\right) \Upsilon_{\boldsymbol{\mu}+\rho}\left(-\bar{\tau} ; C_{\ell}^{2}, \boldsymbol{K} \cdot C_{\ell}\right)}{\sqrt{2 \tau_{2}}} .
$$

\subsection{Modular completion of $\Psi_{\mu}$}

We will treat in this subsection the completion of $\Psi_{\mu}$. Eq. (4.57) related the holomorphic $q$-series $\Psi_{\mu}(\tau)$ to that the second derivative of the function $\Psi_{\mu}(\tau, z)$. To determine the non-holomorphic completion $\widehat{\Psi}_{\boldsymbol{\mu}}(\tau, \bar{\tau})$, we consider the non-holomorphic differential operator

$$
-\frac{1}{4 \pi^{2}}\left(\frac{\partial^{2}}{\partial z^{2}}+\frac{2 \pi m}{\tau_{2}}\right)
$$

on the completion $\widehat{\Psi}_{\mu}(\tau, \bar{\tau}, z, \bar{z})$ of $\Psi_{\mu}(\tau, z)$ (4.55). The differential operator maps a Jacobi form of weight $k$ and index $m$ to a Jacobi form of weight $k+2$ and index $m$. The second non-holomorphic term in (4.107) is required for modularity but does is not relevant for the holomorphic part.

To determine the completion $\widehat{\Psi}_{\boldsymbol{\mu}}(\tau, \bar{\tau}, z, \bar{z})$, we set $z=\beta \tau+\delta$ with $\beta, \delta \in \mathbb{R}$, so that $\beta=\operatorname{Im}(z) / \tau_{2}$. We first note that completing the square gives for a generic vector $V \in \underline{\boldsymbol{\Lambda}}$,

$$
y^{V \cdot \boldsymbol{Q}} q^{-\frac{1}{2} \boldsymbol{Q}^{2}}=q^{\frac{\beta^{2}}{2} V^{2}-\frac{1}{2}(\boldsymbol{Q}-\beta V)^{2}} e^{2 \pi i(V \cdot \boldsymbol{Q}) \delta},
$$

for an arbitrary vector $V$. To write the modular completion, we can treat the three permutations separately. With $C_{1}$ as in (4.71), and $C_{\ell}^{(123)}$ as in (4.72), we find that the 
kernel $(-1)^{a+b-c} F^{*}(123)\left(y^{a+b-c}+y^{-a-b+c}\right)$ is to be completed to

$$
\widehat{F}^{*}(123, y)=\frac{(-1)^{a+b-c}}{4} \sum_{ \pm}\left[1+\sum_{\ell=1}^{3} E_{2}\left(C_{\ell}^{(123)}, C_{\ell+1}^{(123)} ; \sqrt{2 \tau_{2}}\left(\boldsymbol{Q} \mp \beta C_{1}\right)\right)\right] y^{ \pm(a+b-c)} .
$$

Then the completion $\widehat{\Psi}_{\boldsymbol{\mu}}(\tau, \bar{\tau}, z, \bar{z})$ reads,

$$
\widehat{\Psi}_{\boldsymbol{\mu}}(\tau, \bar{\tau}, z, \bar{z})=\sum_{\boldsymbol{Q} \in \underline{\boldsymbol{\Lambda}}_{\boldsymbol{\mu}}^{*}}\left[\widehat{F}^{*}(123, y)+\widehat{F}^{*}(213, y)+\widehat{F}^{*}(132, y)\right] q^{-Q^{2} / 2}
$$

The completion $\widehat{\Psi}_{\boldsymbol{\mu}}(\tau, \bar{\tau}, z, \bar{z})$ transforms as a Jacobi form of weight $b_{2} / 2$ and index $m_{P}$ given by,

$$
m_{P}=-\frac{1}{2} C_{1}^{2}=-P_{1} P_{2} P_{3}-\frac{1}{2} \sum_{\ell=1}^{3} P_{\ell}\left(P_{\ell+1}^{2}+P_{\ell+2}^{2}\right) .
$$

This is symmetric under permutations, and thus also equal to $-C_{2}^{2} / 2=-C_{3}^{2} / 2$.

To write the modular completion, we define the function

$$
\begin{aligned}
G\left(V_{1}, V_{2}, V_{3} ; \boldsymbol{Q}, \tau_{2}\right)= & -\left.\frac{1}{4 \pi^{2}} \partial_{z}^{2}\left(E_{2}\left(V_{1}, V_{2} ; \sqrt{2 \tau_{2}}\left(\boldsymbol{Q}-\beta V_{3}\right)\right) y^{V_{3} \cdot \boldsymbol{Q}}\right)\right|_{z=0} \\
= & \left(V_{3} \cdot \boldsymbol{Q}\right)^{2} E_{2}\left(V_{1}, V_{2} ; \sqrt{2 \tau_{2}} \boldsymbol{Q}\right) \\
& -\left.\frac{1}{4 \pi^{2}} \partial_{z}^{2} E_{2}\left(V_{1}, V_{2} ; \sqrt{2 \tau_{2}}\left(\boldsymbol{Q}-\beta V_{3}\right)\right)\right|_{z=0} \\
& +\left.\frac{1}{2 \pi i}\left(V_{3} \cdot \boldsymbol{Q}\right) \partial_{z} E_{2}\left(V_{1}, V_{2} ; \sqrt{2 \tau_{2}}\left(\boldsymbol{Q}-\beta V_{3}\right)\right)\right|_{z=0} .
\end{aligned}
$$

The limit $\tau_{2} \rightarrow \infty$ is determined by the first term,

$$
\lim _{\tau_{2} \rightarrow \infty} G\left(V_{1}, V_{2}, V_{3} ; \boldsymbol{Q}, \tau_{2}\right)=\left\{\begin{aligned}
\left(V_{3} \cdot \boldsymbol{Q}\right)^{2} \arctan (\alpha), & \text { if } V_{1} \cdot \boldsymbol{Q}=V_{2} \cdot \boldsymbol{Q}=0, \\
\left(V_{3} \cdot \boldsymbol{Q}\right)^{2} \operatorname{sgn}\left(V_{1} \cdot \boldsymbol{Q}\right) \operatorname{sgn}\left(V_{2} \cdot \boldsymbol{Q}\right), & \text { otherwise }
\end{aligned}\right.
$$

with $\alpha$ as in (4.75).

To write the modular completion, we can treat the three permutations separately. We set

$$
\widehat{G}^{*}(123)=\frac{(-1)^{a+b-c}}{4}\left((a+b-c)^{2}+\sum_{\ell=1}^{3} G\left(C_{\ell}^{(123)}, C_{\ell+1}^{(123)}, C_{1} ; \boldsymbol{Q}, \tau_{2}\right)\right),
$$

with $C_{1}$ as in (4.71), and $C_{\ell}^{(123)}$ as in (4.72) The other permutations (213) and (132) give similarly rise to $\widehat{F}^{*}(213)$ and $\widehat{F}^{*}(132)$. We define,

$$
\widehat{g}_{C}\left(\left\{\gamma_{j}\right\} ;\left\{c_{j}^{*}\right\}\right)=\frac{1}{4}\left[\widehat{G}^{*}(123)+\widehat{G}^{*}(213)+\widehat{G}^{*}(132)\right] .
$$

The modular completion $\widehat{\Psi}_{\mu}$ now reads

$$
\widehat{\Psi}_{\boldsymbol{\mu}}(\tau, \bar{\tau})=\sum_{\boldsymbol{Q} \in \underline{\boldsymbol{\Lambda}}_{\mu}^{*}} \widehat{g}_{C}\left(\left\{\gamma_{j}\right\} ;\left\{c_{j}^{*}\right\}\right) q^{-Q^{2} / 2}-\frac{m_{P}}{2 \pi \tau_{2}} \widehat{\Phi}_{\boldsymbol{\mu}}(\tau, \bar{\tau}),
$$


where the last term is due to the $m$-dependent term in (4.107). We let this expression be our final form for $\widehat{\Psi}_{\boldsymbol{\mu}}(\tau, \bar{\tau})$.

Our last task is to determine the constant $A$ in $g_{C}\left(\left\{\gamma_{j}\right\} ;\left\{c_{j}^{*}\right\}\right)$ (2.42). To this end, we consider the $\tau_{2} \rightarrow \infty$ limit of $\widehat{g}_{C}\left(\left\{\gamma_{j}\right\} ;\left\{c_{j}^{*}\right\}\right)$ and require that it reduces to $g_{C}\left(\left\{\gamma_{j}\right\} ;\left\{c_{j}^{*}\right\}\right)$. If we consider the term $F^{*}(123)(a+b-c)^{2}$, we see that only the completion of the term $\operatorname{sgn}(a-c) \operatorname{sgn}(b-c)$ in $g_{C}$ can contribute a non-vanishing difference. Indeed, if we include the other permutations, the only remaining terms in the difference are the equilateral cases with $a=b=c,{ }^{4}$

$$
\begin{aligned}
\lim _{\tau_{2} \rightarrow \infty} \widehat{g}_{C}\left(\left\{\gamma_{j}\right\} ;\left\{c_{j}^{*}\right\}\right)-g_{C}\left(\left\{\gamma_{j}\right\} ;\left\{c_{j}^{*}\right\}\right)=\frac{(-1)^{a}}{4} a^{2} \delta_{a, b} \delta_{b, c} \\
\times \lim _{\tau_{2} \rightarrow \infty}\left(E_{2}\left(C_{a}-C_{c}, C_{b}-C_{c} ; \sqrt{2 \tau_{2}} \boldsymbol{Q}\right)+E_{2}\left(C_{a}-C_{b}, C_{c}-C_{b} ; \sqrt{2 \tau_{2}} \boldsymbol{Q}\right)\right. \\
\left.\left.+E_{2}\left(C_{c}-C_{a}, C_{b}-C_{a} ; \sqrt{2 \tau_{2}} \boldsymbol{Q}\right)-A\right)\right) .
\end{aligned}
$$

Now the sum of $E_{2}$ 's is precisely of the form (4.85), and thus equals 1 . As a result, we find that with $A=1$ the limit vanishes for any choice of Calabi-Yau and charge configurations. This matches perfectly with the physical expectation. Note that the individual values for $E_{2}$ are given by an arctan, and are generically irrational, but that this combination adds up to 1 .

It is quite striking that the values of $E_{2}$ 's precisely confirm the physical expectation. Also in other cases $[30,43]$, the values of the generalized error functions for vanishing arguments has matched with the expectations based on BPS invariants.

\section{Relation to M5-branes and $\mathrm{AdS}_{3} / \mathrm{CFT}_{2}$}

In this brief section, we discuss our findings from the point of view of M-theory and the MSW CFT. We discuss how the partition functions $\widehat{Z}_{P}^{n T}, n>2$, may arise from the 2-dimensional perspective.

The uplift of the D4-branes to M5-branes in M-theory is useful to understand the modular properties of the partition functions [6, 12-14]. The spatial dimensions of the M5-brane are $P \times S_{M}^{1}$, where $P \in H_{4}(X, \mathbb{Z})$ is a four-cycle in the Calabi-Yau threefold and $S_{M}^{1}$ is the M-theory circle. The D2 branes of IIA string theory are realized as excitations of the self-dual 2-form field on M5 brane world volume, while the D0-branes are realized as momenta of the brane system along $S_{M}^{1}$ with radius $R=g_{s} \ell_{s}=\ell_{11}^{3} / \ell_{s}^{2}$ in terms of the string coupling $g_{s}$, string length $\ell_{s}$, and eleven dimensional Planck length $\ell_{11}$. The world volume theory of the M5 brane gives a low energy description of the system, provided gravitational effects can be ignored. This can be ensured by taking the volume of the $\mathrm{CY}_{3}$ to be large, namely $V_{X} / \ell_{11}^{6}$ large but fixed. Supersymmetry of the M5 world volume theory implies that the MSW CFT has $(0,4)$ supersymmetry, and is dual to the near horizon $\mathrm{AdS}_{3}$ geometry $[12,76]$. Bosons of this CFT include, moduli of the divisor $P$ inside $X$, translations along flat $\mathbb{R}^{3}$ and (anti)-chiral scalars coming from reduction of self-dual two-form field in

\footnotetext{
${ }^{4}$ We stress that the equilateral condition $a=b=c$, is the condition on $\boldsymbol{Q}$ to satisfy $C_{a} \cdot \boldsymbol{Q}=C_{b} \cdot \boldsymbol{Q}=C_{c} \cdot \boldsymbol{Q}$, and does not imply equalities among $C_{a}, C_{b}$ and $C_{c}$.
} 
the M5 brane world volume. The number of fields can be determined using geometric data of $X$, when $P$ is a very ample divisor. Consequently the central charges can be determined. The central charge of the left-moving, non-supersymmetric sector is

$$
c_{L}=P^{3}+c_{2}(X) \cdot P,
$$

where $c_{2}(X)$ is the second Chern class of the Calabi-Yau three-fold $X$. Using Cardy's formula, Reference [12] demonstrated that microscopic entropy is in agreement with one loop corrected Bekenstein-Hawking entropy.

As for any CFT, a key feature of the MSW CFT is the modular symmetry. When time direction is compactified, the CFT lives on a 2-torus $T^{2}=S_{M}^{1} \times S_{t}^{1}$. The linear fractional transformation of the complex structure modulus $\tau$ of $T^{2}$ by an element of $\operatorname{SL}(2, \mathbb{Z})$ corresponds to the same torus $T^{2}$. Thus the $\operatorname{SL}(2, \mathbb{Z})$ symmetry, or modular symmetry, appears as a symmetry of the CFT. This is a strong constraint on the degeneracies. Another property of the CFT is the spectral flow of the $\mathrm{U}(1)^{b_{2}}$ current algebra. This leads to a symmetry of degeneracies as function of the charges, such that the partition function can be decomposed as a finite sum of theta series $\times$ modular functions, much as is the case for the attractor partition function in section 3.2.

We proceed by considering the $\mathrm{AdS}_{3}$ dual to the MSW CFT following [27]. The relation of $\lambda$ in (3.14) to the five-dimensional quantities can be understood from four-dimensional Newton's constant. From the IIA perspective, we have

$$
G_{4}=\frac{g_{s}^{2} \ell_{s}^{8}}{V_{X}}=\frac{g_{s}^{2} \ell_{s}^{2}}{\lambda^{3}}
$$

while in terms of the five-dimensional quantities,

$$
G_{4}=\frac{\ell_{5}^{3}}{R}=g_{s}^{2} \ell_{s}^{2} \frac{\ell_{5}^{3}}{R^{3}}
$$

such that one has,

$$
\lambda=\frac{R}{\ell_{5}} .
$$

Starting from five dimensional asymptotically flat geometries with appropriate charges, one takes the decoupling limit as follows: size of M-theory circle $R$ is kept fixed (in absolute units), Calabi-Yau volume is kept finite in units of five dimensional Planck length $\ell_{5}$. In the decoupling limit to $\mathrm{AdS}_{3}, \ell_{5} \rightarrow 0$, hence $\lambda \rightarrow \infty$ by (5.4). Multi-centered geometries where centers have mutual distance of the order of $\ell_{5}^{3} \sim \lambda^{-3}$ or less, survive this limit and go over to asymptotically $\mathrm{AdS}_{3} \times S^{2}$ geometries, with asymptotic moduli fixed to their attractor values. These are the $\lambda$-core geometries mentioned in the introduction, and include centers with non-vanishing individual D6 brane charges, which add up to zero. On the other hand, if the distances between the centers is larger than $\sim \ell_{5}^{3} / R^{2}$ for $\ell_{5} \rightarrow 0$, the energy of their fluctuations diverges and such bound states decouple from the spectrum. As we can see from the Denef equations (2.2), this is for example the case if the centers carry a non-vanishing D4-brane charge with vanishing D6-brane charge. Then the distances between the centers scale as $\ell_{5}$ for non-scaling bound states, and the centers give rise to disconnected $\mathrm{AdS}_{3}$ 
throats in the decoupling limit [27]. On the other hand for scaling solutions, the distance between the centers contains a regime where the centers can come arbitrarily close.

As explained in section 2, the BPS index can be determined using localization with respect to rotation around the $z$-axis, and we can therefore concentrate on collinear solutions to Denef's equations [68]. These collinear solutions admit two branches, one corresponding to centers at finite distances, and the other one is when the centers are nearly coincident. The second branch, sometimes referred to as "deep scaling regime" reproduces pure Higgs states and goes to a single throat in $\ell_{5} \rightarrow 0$ limit [53]. This is in accordance with the expectation that MSW CFT captures the near coincident regime of scaling solutions since these are smoothly connected to the single center black hole. Since $c_{j} \sim \lambda(4.1)$, The separation between the centers at the collinear fixed point is of order $\lambda^{-1} \sim \ell_{5}$, and these therefore decouple from the $\mathrm{AdS}_{3}$ geometry. As a result, these do not appear to be captured by the CFT. This leads us to speculate that the first term on the r.h.s. of (1.1), $\widehat{\mathcal{Z}}_{P}^{T}$, corresponds to the $\mathrm{AdS}_{3} / \mathrm{CFT}_{2}$ partition function while the other terms on the r.h.s. do not.

It is intriguing that the terms due to scaling solutions $\widehat{\mathcal{Z}}_{P}^{n T}$ with $n \geq 3$ do satisfy the restrictive modular transformations as well as the spectral flow symmetry, and it is desirable to understand the origin. We think that these terms can appear after turning on an irrelevant deformation in the $(0,4) \mathrm{CFT}$, away from the conformal fixed point and reversing the attractor flow and $\ell_{5} \rightarrow 0$ limit. While such a deformation does not lead to a finite change in the partition function for $(4,4)$ CFT [54], it seems plausible to us that this can happen with reduced supersymmetry. This deformation is distinguished among other deformations spanning the space of attractor moduli, since this deformation does preserve the spectral flow symmetry which is in general not the case for variations of the Kähler moduli orthogonal to $P$ [25]. We leave a further exploration of these interesting aspects for future work.

\section{Case studies}

To make a suitable choice of a Calabi-Yau threefold, we note that the lattices involved have dimensions linear in $b_{2}$, the second Betti number. Thus the computations are less involved for smaller $b_{2}$. However for the simplest case $b_{2}=1$, there are no cyclic quivers and hence no scaling solutions. ${ }^{5}$ Also, the lattices concerned are positive definite, and therefore do not give rise to mock modular forms. So we settle for the next simplest case $b_{2}=2$.

In order to define quadratic forms on the lattices, one needs the intersection numbers. For a Calabi Yau threefold with $b_{2}=2$, there are $2^{3}=8$ intersection numbers of which only 4 are independent. For the sake of simplicity we shall choose a Calabi Yau threefold $X$, for which all intersection numbers are $\in 2 \mathbb{Z}$ such that Simplification 2 of section 4.1 holds, and for which most of these numbers vanish. To be specific, we choose $X$ to be a $K 3$ fibration over $\mathbb{P}^{1}$. This Calabi-Yau manifold corresponds to Polytope ID \# $=14$ in the online database [77]. $X$ can be realized as a divisor in an ambient toric variety $\mathbb{P}^{3} \times \mathbb{P}^{1}$,

\footnotetext{
${ }^{5}$ Consider triangular quiver to start with. For $b_{2}=1$, electric and magnetic charges are numbers and satisfy the identity $a P_{3}+b P_{1}+c P_{2}=0$. Since $P_{i}>0$ this implies $a, b, c$ can not all have the same sign hence the quiver can not be cyclic. This is easily generalized to any cyclic quiver.
} 
specified by the weight matrix

$$
W=\left(\begin{array}{llllll}
0 & 0 & 0 & 1 & 1 & 0 \\
1 & 1 & 1 & 0 & 0 & 1
\end{array}\right)
$$

$W$ defines the toric variety through the identification on $\mathbb{C}^{6} /\{0\}$

$$
\left(z_{1}, \ldots, z_{6}\right) \sim\left(\lambda^{W_{i 1}} z_{1}, \ldots, \lambda^{W_{i 6}} z_{6}\right), \lambda \in \mathbb{C}^{\star}, i=1,2,
$$

where $z_{1}, \ldots, z_{6}$ are coordinates of $\mathbb{C}^{6}$. Anti-canonical divisor of this toric variety defines the Calabi Yau threefold under consideration. It has the following Hodge numbers

$$
h^{1,1}=2, \quad h^{2,1}=86
$$

and consequently the Euler number is $\chi=2\left(h^{1,1}-h^{2,1}\right)=-168$. Intersection polynomial and second Chern class of $X$ are given respectively by

$$
\begin{aligned}
\text { intersection polynomial } & =4 J_{1} J_{2}^{2}+2 J_{2}^{3}, \\
\text { second Chern class } & =8 J_{1} J_{2}+6 J_{2}^{2},
\end{aligned}
$$

where $J_{i}$ 's are a basis of 2 -forms on $X$ and generators of the Kähler cone. $X$ is favorable, meaning that Kähler forms on $X$ descend from the ambient toric variety. In our notation, the intersection numbers $d_{a b c}$ and second Chern class $c_{2, a}$ read

$$
\begin{array}{rlrl}
d_{111} & =d_{112}=0, & d_{122} & =4, \\
c_{2,1} & =24, & d_{222}=2, & \\
c_{2,2} & =44
\end{array}
$$

and the $d_{a b c}$ for other indices follow by permutation.

\subsection{Charge configuration 1: $P_{1}=P_{2}=(0,1), P_{3}=(1,1)$}

In this example we choose the total magnetic charge $P=(1,3)$ magnetic charges split into 3 centers as

$$
P_{1}=P_{2}=(0,1), \quad P_{3}=(1,1) .
$$

We note that $P_{1,2}$ are irreducible while $P_{3}$ is reducible. Therefore $h_{P_{1,2}, \mu}$ is a weakly holomorphic modular form, while $h_{P_{3}, \mu}$ is a mock modular form. The corresponding central charges of the left-moving sector of the CFT (5.1) are,

$$
c_{L}\left(P_{1,2}\right)=46, \quad c_{L}\left(P_{3}\right)=92, \quad c_{L}(P)=318 .
$$

For the $j$-th center, we have for the innerproduct $D_{j}=d_{a b c} P_{j}^{c}$,

$$
D_{1}=D_{2}=\left(\begin{array}{ll}
0 & 4 \\
4 & 2
\end{array}\right), \quad D_{3}=\left(\begin{array}{ll}
0 & 4 \\
4 & 6
\end{array}\right)
$$

For this choice, $D_{3}^{-1} D_{1}=D_{3}^{-1} D_{2}$ is an integral matrix, such that Simplication 1. of section 4.1 is satisfied, and we can use the results of that section. We have, $D_{1} D_{3}^{-1} D_{1}=$ 
$\left(\begin{array}{cc}0 & 4 \\ 4 & -2\end{array}\right)$ which produces the quadratic form of $\underline{\boldsymbol{\Lambda}}(4.33)$

$$
\underline{D}=\left(\begin{array}{cccc}
0 & 8 & 0 & 4 \\
8 & 0 & 4 & -2 \\
0 & 4 & 0 & 8 \\
4 & -2 & 8 & 0
\end{array}\right), \quad \operatorname{det} \underline{D}=2304
$$

The quadratic form on the lattice of electric charges $\underline{D}^{-1}$ is given by,

$$
\underline{D}^{-1}=\left(\begin{array}{cccc}
-\frac{1}{18} & \frac{1}{6} & \frac{5}{72} & -\frac{1}{12} \\
\frac{1}{6} & 0 & -\frac{1}{12} & 0 \\
\frac{5}{72} & -\frac{1}{12} & -\frac{1}{18} & \frac{1}{6} \\
-\frac{1}{12} & 0 & \frac{1}{6} & 0
\end{array}\right) .
$$

Electric charge vectors $Q_{j} \in \Lambda_{j}^{*}$ have the following form

$$
Q_{j}=\left(\begin{array}{c}
q_{j 1} \\
q_{j 2}
\end{array}\right)
$$

There are $\operatorname{det}\left(D_{1}\right) \operatorname{det}\left(D_{2}\right)=256$ conjugacy classes of the form

$$
\begin{array}{ll}
q_{11}=4 k_{12}+\mu_{11}, & q_{12}=4 k_{11}+2 k_{12}+\mu_{12}, \\
q_{21}=4 k_{22}+\mu_{21}, & q_{22}=4 k_{21}+2 k_{22}+\mu_{22},
\end{array}
$$

where $\mu_{i j} \in\{0,1,2,3\}, k_{i j} \in \mathbb{Z}$. These conjugacy classes have the exchange symmetry between $\mu_{1}, \mu_{2}$. Since $D_{3}$ divides $D_{1}$ and $D_{2}, N_{q}=\operatorname{det}\left(D_{1}\right) \operatorname{det}\left(D_{2}\right)$ (4.35) is the number of conjugacy classes of $\underline{\boldsymbol{\Lambda}}^{*} / \underline{\boldsymbol{\Lambda}}$ for fixed $\mu_{1}+\mu_{2}+\mu_{3}=\mu \in \Lambda^{*} / \Lambda$, since the class $\mu_{3} \in \Lambda_{3}^{*} / \Lambda_{3}$ by the simplification. Using the equations in (4.39), we can write

$$
\begin{aligned}
& \boldsymbol{Q}_{1}=\left(\begin{array}{c}
8 k_{12}+4 k_{22}+\mu_{11}-\mu_{31} \\
8 k_{11}+4 k_{21}-2 k_{22}+\mu_{12}+\mu_{31}-\mu_{32}
\end{array}\right), \\
& \boldsymbol{Q}_{2}=\left(\begin{array}{c}
8 k_{22}+4 k_{12}+\mu_{21}-\mu_{31} \\
8 k_{21}+4 k_{11}-2 k_{12}+\mu_{22}+\mu_{31}-\mu_{32}
\end{array}\right) .
\end{aligned}
$$

We restrict to $\mu=0=\mu_{1}+\mu_{2}+\mu_{3}$, such that

$$
\begin{aligned}
& \boldsymbol{Q}_{1}=\left(\begin{array}{c}
8 k_{12}+4 k_{22}+2 \mu_{11}+\mu_{21} \\
8 k_{11}+4 k_{21}-2 k_{22}+2 \mu_{12}+\mu_{22}-\mu_{11}-\mu_{21}
\end{array}\right), \quad \boldsymbol{\mu}_{1}=\left(\begin{array}{c}
2 \mu_{11}+\mu_{21} \\
2 \mu_{12}+\mu_{22}-\mu_{11}-\mu_{21}
\end{array}\right), \\
& \boldsymbol{Q}_{2}=\left(\begin{array}{c}
8 k_{22}+4 k_{12}+2 \mu_{21}+\mu_{11} \\
8 k_{21}+4 k_{11}-2 k_{12}+2 \mu_{22}+\mu_{12}-\mu_{11}-\mu_{21}
\end{array}\right), \quad \boldsymbol{\mu}_{2}=\left(\begin{array}{c}
2 \mu_{21}+\mu_{11} \\
2 \mu_{22}+\mu_{12}-\mu_{11}-\mu_{21}
\end{array}\right) .
\end{aligned}
$$

List of $\Phi_{\boldsymbol{\mu}}$. We next present explicit $q$-expansions of $\Phi_{\boldsymbol{\mu}}$ for a various choices of $\boldsymbol{\mu}$. All the convergence conditions of the types $C_{i}^{2} C_{i+1}^{2}-\left(C_{i} \cdot C_{i+1}\right)^{2}>0$ and $C_{i}^{2}\left(C_{i-1} \cdot C_{i+1}\right)-$ $\left(C_{i} . C_{i-1}\right) \cdot\left(C_{i} . C_{i+1}\right)<0$ are satisfied in this example along with $C_{j}^{2}>0$. As in explained in section $4.1, \boldsymbol{\mu}$ is fully determined by specifying $\left(\mu_{1}, \mu_{2}, \mu_{3}\right)$ and $\mu=\mu_{1}+\mu_{2}+\mu_{3}$. In the 
following example, we fix $\mu=\mu_{1}=0$ and $\mu_{3}=-\mu_{2}$, and list $\Phi_{\mu}$ for 16 different values of $\mu_{2}$. Out of this set, 10 give rise to different $q$-series. The overall sign of the $q$-expansion is given by $(-1)^{(a+b+c)}=(-1)^{\mu_{11}-\mu_{21}}=(-1)^{\mu_{21}}$. Furthermore, the growth of the coefficients is moderate, $|c(n)| \leq C n^{\alpha}$ for some positive constants $\alpha$ and $C$, which is expected for a holomorphic (mock) modular form of weight 2 .

It can be shown that in most of the sectors we study $\left(\mu_{1}=(0,0)\right)$ there is no contribution to the holomorphic part when the arguments of $E_{2}$ are both zero. When $\mu_{2}=(1, j)$ or $\mu_{2}=(3, j) b$ is odd while $a, c$ are both odd or both even. Under these conditions at least two of $(a+b-c),(a-b+c),(c+b-a)$ cannot vanish. Only in the conjugacy classes $\mu_{2}=(0,0),(0,2),(2,3)$ we find integral $k_{11}, k_{12}, k_{21}, k_{22}$ to satisfy at least two of $(a+b-c),(a-b+c),(c+b-a)$ to vanish. The contributions $A_{\ell}$ are given by

$$
\begin{aligned}
& A_{1}=\frac{2}{\pi} \arctan (-5 / \sqrt{11}), \\
& A_{2}=A_{3}=\frac{2}{\pi} \arctan (-1 / \sqrt{8}),
\end{aligned}
$$

which are all irrational numbers.

For $\mu_{2}=(0,0)$, we have:

$$
\begin{aligned}
\Phi_{\boldsymbol{\mu}}= & 2 q^{6}+4 q^{20}+6 q^{24}+4 q^{30}+4 q^{44}+4 q^{50}+8 q^{52}+2 q^{54}+12 q^{56}+4 q^{60} \\
& +4 q^{64}+4 q^{68}+4 q^{70}+12 q^{80}+2 q^{88}+8 q^{90}+8 q^{92}+8 q^{94}+14 q^{96}+16 q^{100} \\
& +\frac{\left(A_{1}+A_{2}+A_{3}+1\right)}{2}+\left(A_{1}+A_{2}+A_{3}+3\right)\left(q^{8}+q^{32}+q^{72}\right)+\ldots
\end{aligned}
$$

For $\mu_{2}=(0,1)$, we have:

$$
\begin{aligned}
\Phi_{\boldsymbol{\mu}}= & q^{5}\left(1+q+2 q^{12}+2 q^{15}+2 q^{17}+2 q^{20}+q^{22}+2 q^{23}+q^{27}+3 q^{28}+q^{30}+2 q^{35}+2 q^{38}\right. \\
& +3 q^{40}+2 q^{42}+4 q^{45}+3 q^{46}+3 q^{49}+4 q^{51}+2 q^{52}+2 q^{53}+3 q^{54}+2 q^{55}+2 q^{57}+3 q^{60} \\
& +2 q^{61}+2 q^{63}+q^{64}+2 q^{65}+5 q^{69}+6 q^{70}+2 q^{71}+2 q^{73}+3 q^{75}+2 q^{78}+2 q^{79}+2 q^{81} \\
& \left.+q^{82}+2 q^{83}+6 q^{84}+2 q^{86}+3 q^{87}+4 q^{88}+5 q^{90}+4 q^{91}+2 q^{92}+6 q^{93}+2 q^{94}+2 q^{95}\right)+\ldots
\end{aligned}
$$

For $\mu_{2}=(0,2)$, we have:

$$
\begin{aligned}
\Phi_{\boldsymbol{\mu}}= & 2 q^{14}+2 q^{20}+2 q^{24}+2 q^{26}+2 q^{32}+6 q^{36}+4 q^{40}+4 q^{42}+2 q^{44}+4 q^{48} \\
& +10 q^{56}+6 q^{60}+4 q^{62}++4 q^{68}+4 q^{70}+8 q^{72}+8 q^{76}+10 q^{80}+4 q^{82}+4 q^{86} \\
& +8 q^{88}+4 q^{92}+4 q^{96}+6 q^{98}+8 q^{100}+\left(A_{1}+1\right)\left(q^{50}+q^{54}+q^{62}+q^{74}+q^{90}\right)+\ldots
\end{aligned}
$$

For $\mu_{2}=(1,0)$, we have:

$$
\begin{aligned}
\Phi_{\mu}= & -2 q^{29 / 4}\left(1+q^{4}+q^{12}+q^{17}+q^{18}+q^{19}+2 q^{21}+q^{25}+q^{31}+q^{37}+2 q^{40}+q^{42}+q^{43}\right. \\
& +2 q^{45}+q^{48}+2 q^{49}+3 q^{50}+q^{52}+q^{53}+2 q^{54}+q^{55}+q^{56}+q^{57}+2 q^{60} \\
& +q^{66}+2^{71}+2 q^{74}+q^{75}+q^{76}+3 q^{79}+q^{80}+2 q^{81}+q^{82}+3 q^{84}+q^{85}+q^{86} \\
& \left.+3 q^{87}+2 q^{90}+3 q^{91}+3 q^{92}\right)+\ldots
\end{aligned}
$$


For $\mu_{2}=(1,1)$, we have:

$$
\begin{aligned}
\Phi_{\boldsymbol{\mu}}= & -2 q^{83 / 4}\left(1+q^{3}+q^{5}+q^{8}+q^{9}+q^{10}+q^{13}+q^{16}+q^{22}+q^{23}+q^{24}+q^{26}+3 q^{31}\right. \\
& +2 q^{33}+q^{34}+q^{35}+q^{36}+2 q^{38}+q^{39}+q^{40}+q^{41}+2 q^{42}+q^{44}+q^{45}+q^{46}+q^{49}+2 q^{51} \\
& +2 q^{54}+q^{57}+q^{58}+2 q^{59}+2 q^{60}+2 q^{61}+q^{68}+q^{69}+q^{70}+2 q^{71}+4 q^{72}+3 q^{72}+q^{74} \\
& \left.++2 q^{75}+q^{76}+q^{77}+3 q^{78}+q^{79}\right)+\ldots
\end{aligned}
$$

For $\mu_{2}=(1,2)$, we have:

$$
\begin{aligned}
\Phi_{\boldsymbol{\mu}}= & -2 q^{45 / 4}\left(1+2 q^{8}+q^{15}+q^{18}+q^{21}+q^{23}+2 q^{27}+q^{28}+q^{29}+q^{32}+2 q^{35}+q^{39}+q^{41}\right. \\
& +2 q^{43}+q^{44}+q^{46}+2 q^{48}+q^{50}+q^{52}+q^{54}+q^{56}+3 q^{57}+q^{58}+2 q^{62}+3 q^{63}+3 q^{65} \\
& \left.+q^{66}+q^{68}+q^{70}+2 q^{72}+q^{73}+2 q^{74}+q^{75}+3 q^{80}+q^{82}+2 q^{85}+q^{86}+q^{87}+q^{88}\right)+\ldots
\end{aligned}
$$

For $\mu_{2}=(1,3)$, we have:

$$
\begin{aligned}
\Phi_{\boldsymbol{\mu}}= & -2 q^{15 / 4}\left(1+q+q^{10}+q^{13}+q^{17}+q^{18}+q^{19}+q^{21}+q^{22}+2 q^{32}+q^{35}+q^{39}\right. \\
& +2 q^{40}+q^{42}+q^{45}+q^{47}+q^{50}+2 q^{51}+q^{53}+3 q^{54}+q^{56}+2 q^{59}+q^{63}+q^{65}+2 q^{66} \\
& +2 q^{68}+q^{71}+q^{72}+q^{74}+q^{75}+q^{76}+3 q^{77}+q^{79}+2 q^{80}+2 q^{83}+q^{84}+2 q^{85}+q^{86} \\
& \left.+q^{87}+q^{88}+2 q^{90}+2 q^{92}+3 q^{93}+2 q^{95}\right)+\ldots
\end{aligned}
$$

For $\mu_{2}=(2,0)$, we have:

$$
\begin{aligned}
\Phi_{\boldsymbol{\mu}}= & 2\left(q^{9}+q^{15}+q^{19}+2 q^{27}+2 q^{29}+q^{33}+q^{35}+q^{43}+4 q^{45}+4 q^{53}+2 q^{55}+q^{57}+q^{59}\right. \\
& +2 q^{61}+2 q^{63}+3 q^{65}+q^{67}+2 q^{71}+q^{75}+4 q^{77}+2 q^{81}+3 q^{83}+2 q^{87}+q^{89}+5 q^{91} \\
& \left.+q^{93}+6 q^{95}+q^{97}+2 q^{99}\right)+\ldots
\end{aligned}
$$

For $\mu_{2}=(2,1)$, we have:

$$
\begin{aligned}
\Phi_{\boldsymbol{\mu}}= & 2 q^{25}\left(2+2 q+q^{2}+q^{4}+2 q^{15}+q^{16}+2 q^{18}+q^{22}+2 q^{25}+2 q^{29}+4 q^{32}+3 q^{34}+4 q^{37}\right. \\
& +2 q^{39}+q^{40}+2 q^{41}+q^{44}+q^{46}+2 q^{51}+2 q^{55}+q^{56}+2 q^{59}+4 q^{61}+3 q^{62}+2 q^{65}+2 q^{66} \\
& \left.+2 q^{68}+4 q^{70}+2 q^{73}+2 q^{74}+2 q^{75}\right)+\ldots
\end{aligned}
$$

For $\mu_{2}=(2,3)$, we have:

$$
\begin{aligned}
\Phi_{\boldsymbol{\mu}}= & 2 q^{5}\left(1+q^{6}+q^{9}+q^{15}+q^{19}+2 q^{20}+q^{27}+q^{28}+q^{33}+2 q^{34}+q^{37}+q^{40}+q^{42}+2 q^{47}+3 q^{51}\right. \\
& +q^{54}+2 q^{55}+2 q^{56}+q^{60}+q^{63}+2 q^{64}+q^{66}+q^{69}+2 q^{70}+4 q^{71}+2 q^{73}+q^{75}+2 q^{78}+3 q^{81} \\
& \left.+2 q^{84}+2 q^{85}+2 q^{88}+4 q^{90}+3 q^{91}+2 q^{92}+3 q^{94}\right)+\left(A_{3}+1\right) q^{2}\left(1+q^{16}+q^{48}+q^{96}\right)+\ldots
\end{aligned}
$$


List of $\Psi_{\mu}$. Similarly to the $\Phi_{\boldsymbol{\mu}}$, we list the $q$-expansion of $\Psi_{\boldsymbol{\mu}}$ for a various choices of $\boldsymbol{\mu}$. In these examples, we keep $\mu=\mu_{1}=0$ and $\mu_{3}=-\mu_{2}$, and list $\Psi_{\mu}$ for 16 different values of $\mu_{2}$. Out of these 16 only 10 give rise to different $q$-series. The factor $(-1)^{(a+b+c)}=$ $(-1)^{\mu_{11}-\mu_{21}}=(-1)^{\mu_{21}}$ fixes the overall sign of the whole series. Comparison of the $\Phi_{\boldsymbol{\mu}}$ and $\Psi_{\mu}$ demonstrates that for small powers of $q$, scaling solutions can be present kinematically, but that the number of bound states vanishes quantum mechanically. We again observe a moderate, polynomial growth of the coefficients, as expected for a (mock) modular form of weight 4 .

For $\mu_{2}=(0,0)$, we have:

$$
\Psi_{\boldsymbol{\mu}}=16 q^{30}\left(1+2 q^{22}+4 q^{34}+q^{40}+2 q^{60}+8 q^{62}+2 q^{64}+4 q^{70}\right)+\ldots
$$

For $\mu_{2}=(0,1)$, we have:

$$
\begin{aligned}
\Psi_{\boldsymbol{\mu}}= & 2 q^{17}\left(1+q^{5}+q^{8}+q^{11}+q^{16}+q^{23}+q^{26}+q^{28}+4 q^{30}+q^{33}+q^{34}+9 q^{37}+5 q^{39}+q^{40}\right. \\
& +q^{41}+q^{42}+4 q^{43}+4 q^{45}+4 q^{48}+q^{49}+9 q^{51}+q^{53}+q^{57}+6 q^{58}+q^{59}+q^{61}+q^{63}+9 q^{66} \\
& \left.+4 q^{67}+16 q^{69}+q^{71}+5 q^{72}+16 q^{74}+q^{75}+13 q^{76}+26 q^{78}+q^{79}+q^{80}+13 q^{81}+q^{82}+q^{83}\right) \\
& +\ldots
\end{aligned}
$$

For $\mu_{2}=(0,2)$, we have:

$$
\begin{aligned}
\Psi_{\boldsymbol{\mu}}= & 16 q^{36}\left(1+q^{4}+q^{12}+2 q^{20}+q^{24}+q^{32}+q^{34}+2 q^{36}+2 q^{40}+q^{44}+q^{46}+2 q^{52}+4 q^{56}\right. \\
& \left.+4 q^{62}+2 q^{64}\right)+\ldots
\end{aligned}
$$

For $\mu_{2}=(1,0)$, we have:

$$
\begin{aligned}
\Psi_{\boldsymbol{\mu}}= & -\frac{q^{29 / 4}}{2}\left(1+q^{4}+q^{12}+q^{17}+q^{18}+q^{19}+10 q^{21}+9 q^{25}+q^{31}+q^{37}+10 q^{40}+q^{42}+q^{43}\right. \\
& +34 q^{45}+q^{48}+2 q^{49}+27 q^{50}+q^{52}+q^{53}+50 q^{54}+q^{55}+q^{56}+q^{57}+58 q^{60}+9 q^{66}+2 q^{71} \\
& +q^{74}+9 q^{75}+q^{78}+51 q^{79}+25 q^{80}+10 q^{81}+q^{82}+107 q^{84}+25 q^{85}+49 q^{86}+43 q^{87}+26 q^{90} \\
& \left.+107 q^{91}+51 q^{92}\right)+\ldots
\end{aligned}
$$

For $\mu_{2}=(1,1)$, we have:

$$
\begin{aligned}
\Psi_{\mu}= & -\frac{q^{83 / 4}}{2}\left(9+9 q^{3}+q^{5}+q^{8}+q^{9}+q^{10}+9 q^{13}+q^{16}+9 q^{22}+q^{23}+9 q^{24}+q^{26}+19 q^{31}\right. \\
& +34 q^{33}+9 q^{34}+25 q^{35}+9 q^{36}+26 q^{38}+9 q^{39}+25 q^{40}+q^{41}+10 q^{42}+25 q^{44}+9 q^{45} \\
& +25 q^{46}+q^{49}+2 q^{51}+10 q^{54}+9 q^{57}+9 q^{58}+2 q^{59}+18 q^{60}+34 q^{61}+q^{68}+9 q^{69}+49 q^{70} \\
& \left.+74 q^{71}+140 q^{72}+131 q^{73}+q^{74}+18 q^{75}+q^{76}+49 q^{77}+43 q^{78}+25 q^{79}\right)+\ldots
\end{aligned}
$$

For $\mu_{2}=(1,2)$, we have:

$$
\begin{aligned}
\Psi_{\mu}= & -\frac{q^{45 / 4}}{2}\left(1+2 q^{8}+q^{15}+q^{18}+9 q^{21}+9 q^{23}+2 q^{27}+q^{28}+9 q^{29}+q^{32}+18 q^{35}+9 q^{39}\right. \\
& +9 q^{41}+26 q^{43}+9 q^{44}+25 q^{46}+10 q^{47}+9 q^{50}+q^{52}+9 q^{54}+9 q^{56}+19 q^{57}+25 q^{58}+10 q^{62} \\
& +75 q^{64}+43 q^{65}+9 q^{66}+9 q^{68}+q^{70}+10 q^{72}+q^{73}+18 q^{74}+q^{75}+131 q^{80}+49 q^{82} \\
& \left.+50 q^{85}+44 q^{86}+49 q^{87}+25 q^{88}\right)+\ldots
\end{aligned}
$$


For $\mu_{2}=(1,3)$, we have:

$$
\begin{aligned}
\Psi_{\mu}= & -\frac{q^{15 / 4}}{2}\left(1+q+q^{10}+q^{13}+q^{17}+q^{18}+q^{19}+9 q^{21}+q^{22}+10 q^{32}+q^{35}+25 q^{39}+2 q^{40}\right. \\
& +q^{42}+q^{45}+q^{47}+9 q^{50}+50 q^{51}+q^{53}+27 q^{54}+9 q^{56}+10 q^{59}+q^{63}+9 q^{65}+10 q^{66} \\
& +50 q^{68}+q^{71}+25 q^{72}+q^{74}+9 q^{75}+q^{76}+83 q^{77}+q^{79}+10 q^{80}+10 q^{83}+25 q^{84} \\
& \left.+26 q^{85}+49 q^{86}+q^{87}+q^{88}+26 q^{90}+10 q^{92}+139 q^{93}+34 q^{95}\right)+\ldots
\end{aligned}
$$

For $\mu_{2}=(2,0)$, we have:

$$
\begin{aligned}
\Psi_{\mu}= & 2 q^{9}\left(1+q^{6}+q^{10}+2 q^{18}+2 q^{20}+q^{24}+q^{26}+q^{34}+4 q^{36}+12 q^{44}+2 q^{46}+q^{48}+9 q^{50}+2 q^{52}\right. \\
& +10 q^{54}+3 q^{56}+2 q^{58}+10 q^{62}+q^{66}+4 q^{68}+18 q^{72}+11 q^{74}+2 q^{78}+q^{80}+29 q^{82}+q^{84} \\
& \left.+46 q^{86}+q^{88}+10 q^{90}\right)+\ldots
\end{aligned}
$$

For $\mu_{2}=(2,1)$, we have:

$$
\begin{aligned}
\Psi_{\boldsymbol{\mu}}= & 4 q^{25}\left(1+q+q^{15}+q^{18}+4 q^{25}+4 q^{29}+5 q^{32}+4 q^{34}+8 q^{37}+q^{39}+q^{41}+q^{51}+4 q^{55}\right. \\
& \left.+4 q^{59}+5 q^{61}+4 q^{62}+4 q^{65}+q^{66}+16 q^{68}+16 q^{70}+9 q^{73}+9 q^{74}+4 q^{75}\right)+\ldots
\end{aligned}
$$

For $\mu_{2}=(2,3)$, we have:

$$
\begin{aligned}
\Psi_{\boldsymbol{\mu}}= & 4\left(q^{25}+q^{39}+q^{52}+9 q^{56}+q^{61}+4 q^{69}+q^{75}+17 q^{76}+4 q^{83}+9 q^{89}+q^{90}+q^{93}\right. \\
& \left.++5 q^{95}+q^{96}+4 q^{97}+25 q^{99}\right)+\ldots
\end{aligned}
$$

\subsection{Charge configuration 2: $P_{1}=(1,0), P_{2}=P_{3}=(0,1)$}

In this example the total magnetic charge is $P=(1,2)$ split into three different centers as $P_{1}=(1,0), P_{2}=P_{3}=(0,1)$. The charge $P_{1}$ corresponds to the K3 fiber. The CFT central charge (5.1) of $P_{2,3}$ is given above, while we have for $P_{1}$ and $P$,

$$
c_{L}\left(P_{1}\right)=24, \quad c_{L}(P)=176 .
$$

In the $j$-th center, the quadratic form $D_{j}=d_{a b c} P_{j}^{c}$ is given by

$$
D_{2}=D_{3}=\left(\begin{array}{ll}
0 & 4 \\
4 & 2
\end{array}\right), \quad D_{1}=\left(\begin{array}{ll}
0 & 0 \\
0 & 4
\end{array}\right)
$$

Note that $D_{1}$ is a singular matrix, and we only keep the direction with the non-vanishing eigenvalue. The partition functions for $P_{1}$ can be determined using Noether-Lefshetz theory $[19,21,78]$. On the other hand, the modular properties are in this case very restrictive, and determine the partition function with a little further inpute. First, the lowest exponent of $h_{P, \mu}$ is $-c_{L} / 24=-1$. Therefore, each of $h_{P, \mu}$ has a term with a negative coefficient, and the vector space of weakly holomorphic vector-valued modular forms is 3 -dimensional. We can fix the coefficients following [13, 79]: the coefficient for $\mu=0$ is the Euler number of the linear system of the divisor, which is $c_{2} \cdot P_{1} / 12=2$. Thus the 
linear system is a $\mathbb{P}^{1}$. For $\mu=1$ and 2 , we need to require that the divisor contains a given curve class $\beta \in H^{2}(X, \mathbb{Z})$, and turn on a world volume flux through this curve. This gives two complex constraints, however since the linear system is a $\mathbb{P}^{1}$, there are generically no solutions. As a result, the coefficients of the potential polar terms in $h_{P_{1}, 1}$ and $h_{P_{1}, 2}$ vanish. This fixes $h_{P_{1}, \mu}$ to be the weight $-3 / 2$ modular form,

$$
h_{P_{1}, \mu}(\tau)=\frac{2 f_{P_{1}, \mu}(\tau)}{\eta(\tau)^{24}},
$$

with

$$
\begin{aligned}
f_{P_{1}, \mu}(\tau)= & \frac{11}{18} E_{4}(\tau) E_{6}(\tau) \Theta_{\mu}(\tau) \\
& -6 E_{4}(\tau)^{2} D_{1 / 2} \Theta_{\mu}(\tau) \\
& +16 E_{6}(\tau) D_{5 / 2} D_{1 / 2} \Theta_{\mu}(\tau),
\end{aligned}
$$

with $\Theta_{\mu}(\tau)=\sum_{n \in 4 \mathbb{Z}+\mu} q^{n^{2} / 8}, D_{k}$ the modular derivative acting on weight $k$ modular forms, $D_{k}=\frac{1}{2 \pi i} \frac{d}{d \tau}-\frac{k}{12} E_{2}(\tau)$, and $E_{\ell}$ the Eisenstein series of weight $\ell$. The $f_{P_{1}, \mu}$ also appeared in the context of Gromov-Witten theory $[78,80]$. Finally since $P_{1}^{3}=0, h_{n P_{1}, \mu}$ is also expected to be a weakly holomorphic modular form (rather than a mock modular form), and given in terms of Hecke transformations of $(6.16)$ [21, 40].

Returning to our original problem of $\Phi_{\mu}$ and $\Psi_{\boldsymbol{\mu}}$, note that we have for the products of quadratic forms $D_{1} D_{3}^{-1}=\left(\begin{array}{ll}0 & 0 \\ 1 & 0\end{array}\right), D_{1} D_{3}^{-1} D_{1}=\left(\begin{array}{ll}0 & 0 \\ 0 & 0\end{array}\right), D_{2} D_{3}^{-1} D_{1}=D_{1} D_{3}^{-1} D_{2}=D_{1}$ which produces the quadratic form (4.33),

$$
\underline{D}=\left(\begin{array}{llll}
0 & 0 & 0 & 0 \\
0 & 4 & 0 & 4 \\
0 & 0 & 0 & 8 \\
0 & 4 & 8 & 4
\end{array}\right) \text {. }
$$

Excluding the null direction the determinant of $\underline{D}=-256$. The quadratic form on the lattice of electric charge lattice is given by the pseudo-inverse of $\underline{D}$ as follows:

$$
\underline{D}^{-1}=\left(\begin{array}{cccc}
0 & 0 & 0 & 0 \\
0 & \frac{1}{4} & -\frac{1}{8} & 0 \\
0 & -\frac{1}{8} & 0 & \frac{1}{8} \\
0 & 0 & \frac{1}{8} & 0
\end{array}\right) \text {. }
$$

Electric charge vectors $Q_{j} \in \Lambda_{j}^{*}$ have the following form

$$
Q_{1}=\left(\begin{array}{c}
0 \\
q_{12}
\end{array}\right), \quad Q_{2}=\left(\begin{array}{c}
q_{21} \\
q_{22}
\end{array}\right) .
$$

There are 64 conjugacy classes of the form

$$
\begin{aligned}
& q_{12}=4 k_{12}+\mu_{12}, \\
& q_{21}=4 k_{22}+\mu_{21}, \quad q_{22}=4 k_{21}+2 k_{22}+\mu_{22},
\end{aligned}
$$


where $\mu_{i j} \in\{0,1,2,3\}, k_{i j} \in \mathbb{Z}$. These are $N_{q}=64$ (4.35) conjugacy classes for fixed $\mu$.

Using equations in (4.39), we get

$$
\begin{aligned}
\boldsymbol{Q}_{1} & =\left(\begin{array}{c}
0 \\
4 k_{12}+4 k_{22}+\mu_{12}-\mu_{31}
\end{array}\right) \\
\boldsymbol{Q}_{2} & =\left(\begin{array}{c}
8 k_{22}+\mu_{21}-\mu_{31} \\
8 k_{21}+4 k_{22}+4 k_{12}+\mu_{22}-\mu_{32}
\end{array}\right) .
\end{aligned}
$$

When $\mu_{1}+\mu_{2}+\mu_{3}=0$, we have,

$$
\begin{array}{ll}
\boldsymbol{Q}_{1}=\left(\begin{array}{c}
0 \\
4 k_{12}+4 k_{22}+\mu_{12}+\mu_{21}
\end{array}\right), & \boldsymbol{\mu}_{1}=\left(\begin{array}{c}
0 \\
\mu_{12}+\mu_{21}
\end{array}\right), \\
\boldsymbol{Q}_{2}=\left(\begin{array}{c}
8 k_{22}+2 \mu_{21} \\
8 k_{21}+4 k_{22}+4 k_{12}+2 \mu_{22}+\mu_{12}
\end{array}\right), & \boldsymbol{\mu}_{2}=\left(\begin{array}{c}
2 \mu_{21} \\
2 \mu_{22}+\mu_{12}
\end{array}\right) .
\end{array}
$$

List of $\Phi_{\mu}$. We list $\Phi_{\mu}$ for a various choices of $\boldsymbol{\mu}$. For this choice of charges, we find that some of the inequalities for convergence are sometimes saturated. This however do not give rise to divergences in the $q$ series. As in explained in section $4.1, \boldsymbol{\mu}$ is fully determined by specifying $\left(\mu_{1}, \mu_{2}, \mu_{3}\right)$ and $\mu=\mu_{1}+\mu_{2}+\mu_{3}$. The overall sign of the $q$-expansion is given by $(-1)^{a+b+c}=(-1)^{\mu_{12}}$. The contributions $A_{\ell}$ are for this example

$$
A_{1}=A_{2}=A_{3}=\frac{2}{\pi} \arctan (-3 / 4),
$$

which is an irrational number.

For $\mu_{1}=\mu_{2}=0$, we have:

$$
\begin{aligned}
\Phi_{\boldsymbol{\mu}}= & 4 q^{6}+4 q^{24}+12 q^{30}+6 q^{40}+4 q^{54}+12 q^{64}+12 q^{70}+12 q^{78}+12 q^{88}+4 q^{96} \\
& +\frac{\left(A_{1}+A_{2}+A_{3}+1\right)}{2}+\left(A_{1}+A_{2}+A_{3}+3\right)\left(q^{8}+q^{32}+q^{72}\right)+\ldots
\end{aligned}
$$

For $\mu_{1}=(0,0), \mu_{2}=(1,0)$, we have:

$$
\begin{aligned}
\Phi_{\boldsymbol{\mu}}= & 2 q^{33 / 8}\left(1+q^{4}+q^{7}+q^{16}+2 q^{21}+q^{22}+q^{24}+q^{30}+3 q^{31}+q^{34}+q^{41}+q^{42}+q^{44}\right. \\
& +2 q^{53}+q^{54}+q^{56}+2 q^{58}+q^{60}+2 q^{66}+4 q^{67}+q^{72}+2 q^{74}+q^{76}+2 q^{77}+2 q^{81} \\
& \left.+q^{82}+q^{84}+q^{91}+q^{92}+2 q^{93}\right)+\frac{\left(A_{3}+1\right)}{2} q^{1 / 8}\left(1+q^{6}+q^{10}+q^{28}+q^{36}+q^{66}+q^{78}\right)
\end{aligned}
$$

For $\mu_{1}=(0,0), \mu_{2}=(2,0)$, we have:

$$
\begin{aligned}
\Phi_{\mu}= & 2\left(\frac{1}{2} q^{5 / 2}+q^{21 / 2}+q^{29 / 2}+q^{33 / 2}+q^{41 / 2}+\frac{3}{2} q^{45 / 2}+q^{61 / 2}+q^{65 / 2}+q^{77 / 2}+2 q^{81 / 2}+q^{85 / 2}\right. \\
& +q^{89 / 2}+3 q^{101 / 2}+2 q^{105 / 2}+q^{109 / 2}+q^{117 / 2}+q^{121 / 2}+\frac{1}{2} q^{125 / 2}+2 q^{129 / 2}+q^{133 / 2}+q^{145 / 2} \\
& \left.+q^{149 / 2}+q^{153 / 2}+2 q^{157 / 2}+q^{161 / 2}+q^{165 / 2}+2 q^{173 / 2}+q^{181 / 2}+4 q^{185 / 2}+2 q^{189 / 2}\right) \\
& +q^{1 / 2} \frac{\left(A_{3}+1\right)}{2}\left(1+q^{4}+q^{12}+q^{24}+q^{40}\right)+\ldots
\end{aligned}
$$


For $\mu_{1}=(0,0), \mu_{2}=(3,0)$, we have:

$$
\begin{aligned}
\Phi_{\boldsymbol{\mu}}= & 2 q^{105 / 8}\left(2+q^{5}+q^{6}+q^{13}+q^{14}+2 q^{24}+q^{30}+2 q^{31}+2 q^{33}+q^{34}+q^{38}+q^{40}+q^{43}+2 q^{45}\right. \\
& \left.+q^{46}+q^{52}+3 q^{55}+2 q^{60}+q^{70}+2 q^{71}+2 q^{73}+q^{74}+q^{76}+2 q^{78}+2 q^{82}+q^{84}+q^{86}\right) \\
& +\frac{\left(A_{3}+1\right)}{2} q^{9 / 8}\left(1+q^{2}+q^{14}+q^{20}+q^{44}+q^{54}+q^{90}\right)+\ldots
\end{aligned}
$$

For $\mu_{1}=(0,0), \mu_{2}=(0,1)$, we have:

$$
\begin{aligned}
\Phi_{\boldsymbol{\mu}}= & 2\left(q^{4}+q^{8}+q^{12}+q^{20}+q^{24}+2 q^{26}+q^{28}+2 q^{34}+2 q^{36}+q^{40}+2 q^{46}+q^{48}+q^{56}+2 q^{58}\right. \\
& \left.+2 q^{60}+2 q^{64}+4 q^{70}+2 q^{72}+2 q^{76}+q^{80}+2 q^{82}+2 q^{84}+2 q^{86}+q^{88}+2 q^{96}+2 q^{98}+q^{100}\right)+\ldots
\end{aligned}
$$

For $\mu_{1}=(0,0), \mu_{2}=(0,2)$, we have:

$$
\begin{aligned}
\Phi_{\boldsymbol{\mu}}= & 2\left(q^{10}+2 q^{16}+2 q^{22}+2 q^{32}+2 q^{38}+2 q^{42}+2 q^{48}+4 q^{52}+2 q^{58}+2 q^{62}+2 q^{66}+4 q^{76}\right. \\
& \left.+2 q^{80}+2 q^{82}+3 q^{90}+4 q^{94}+2 q^{96}\right)+q^{2}\left(A_{3}+1\right)\left(1+q^{16}+q^{48}+q^{96}\right)+\ldots
\end{aligned}
$$

For $\mu_{1}=(0,1), \mu_{2}=(1,0)$, we have:

$$
\begin{aligned}
\Phi_{\boldsymbol{\mu}}= & -2 q^{19 / 4}\left(1+q^{2}+q^{6}+q^{17}+2 q^{21}+q^{23}+q^{24}+q^{26}+q^{27}+q^{29}+q^{33}+q^{34}+q^{38}+q^{39}\right. \\
& +q^{46}+q^{52}+q^{54}+q^{56}+q^{57}+q^{58}+q^{61}+2 q^{62}+2 q^{64}+q^{66}+2 q^{68}+q^{69}+q^{71}+q^{72} \\
& \left.+q^{75}+q^{76}+q^{77}+q^{78}+q^{81}+q^{82}+q^{86}+q^{87}+q^{88}+q^{89}+q^{90}+q^{95}\right)+\ldots
\end{aligned}
$$

For $\mu_{1}=(0,1), \mu_{2}=(2,0)$, we have:

$$
\begin{aligned}
\Phi_{\boldsymbol{\mu}}= & -2 q^{71 / 8}\left(1+q^{5}+q^{9}+q^{12}+q^{16}+q^{21}+q^{25}+q^{26}+q^{28}+q^{30}+q^{35}+q^{36}+q^{39}+q^{41}+q^{43}\right. \\
& +q^{45}+q^{47}+q^{48}+q^{50}+2 q^{54}+q^{58}+q^{60}+q^{63}+q^{65}+q^{67}+q^{68}+q^{69}+q^{71}+q^{72}+q^{75} \\
& \left.+q^{76}+q^{78}+q^{80}+q^{81}+q^{82}+q^{86}+q^{88}+q^{89}+q^{90}\right)+\ldots
\end{aligned}
$$

For $\mu_{1}=(0,0), \mu_{2}=(1,1)$, we have:

$$
\begin{aligned}
\Phi_{\mu}= & q^{45 / 8}\left(3+2 q^{4}+q^{10}+4 q^{18}+2 q^{22}+2 q^{23}+4 q^{25}+2 q^{26}+2 q^{30}+4 q^{35}+2 q^{36}+2 q^{43}+4 q^{48}\right. \\
& +2 q^{56}+4 q^{57}+4 q^{59}+4 q^{60}+2 q^{62}+4 q^{66}+2 q^{68}+q^{70}+4 q^{71}+2 q^{72}+4 q^{73}+2 q^{74} \\
& \left.+4 q^{82}+8 q^{85}+4 q^{90}+2 q^{92}\right)+\ldots
\end{aligned}
$$

For $\mu_{1}=(0,0), \mu_{2}=(1,3)$, we have:

$$
\begin{aligned}
\Phi_{\boldsymbol{\mu}}= & q^{5 / 8}\left(1+2 q^{2}+2 q^{10}+2 q^{13}+2 q^{14}+2 q^{16}+2 q^{20}+4 q^{21}+q^{30}+2 q^{32}+4 q^{39}+2 q^{40}+4 q^{42}\right. \\
& +2 q^{44}+3 q^{50}+8 q^{51}+2 q^{54}+4 q^{56}+4 q^{63}+2 q^{64}+2 q^{66}+2 q^{73}+4 q^{77}+2 q^{78} \\
& \left.+2 q^{80}+4 q^{84}+2 q^{86}+2 q^{87}+2 q^{90}+4 q^{92}+8 q^{93}\right)+\ldots
\end{aligned}
$$

For $\mu_{1}=(0,0), \mu_{2}=(2,1)$, we have:

$$
\begin{aligned}
\Phi_{\boldsymbol{\mu}}= & 4 q^{15 / 2}\left(1+q^{12}+q^{20}+q^{24}+2 q^{28}+q^{40}+2 q^{48}+q^{52}+q^{60}+3 q^{64}+2 q^{72}+2 q^{76}\right. \\
& \left.+q^{80}+2 q^{92}\right)+\ldots
\end{aligned}
$$


For $\mu_{1}=(0,0), \mu_{2}=(2,3)$, we have:

$$
\Phi_{\boldsymbol{\mu}}=4 q^{3 / 2}\left(1+q^{12}+3 q^{16}+q^{36}+3 q^{44}+3 q^{48}+3 q^{56}+q^{72}+3 q^{84}+3 q^{92}+3 q^{96}\right)+\ldots
$$

For $\mu_{1}=(0,1), \mu_{2}=(0,0)$, we have:

$$
\begin{aligned}
\Phi_{\boldsymbol{\mu}}= & -2 q^{39 / 8}\left(1+q^{2}+q^{3}+q^{17}+q^{21}+2 q^{22}+q^{24}+q^{26}+q^{27}+2 q^{28}+2 q^{33}+q^{39}+q^{46}+q^{52}\right. \\
& +2 q^{55}+q^{57}+2 q^{60}+q^{61}+2 q^{63}+q^{64}+q^{66}+q^{67}+q^{68}+4 q^{70}+2 q^{76}+q^{78}+2 q^{79}+2 q^{81} \\
& \left.+q^{87}+2 q^{89}+q^{95}\right)+\ldots
\end{aligned}
$$

For $\mu_{1}=(0,1), \mu_{2}=(1,1)$, we have:

$$
\begin{aligned}
\Phi_{\boldsymbol{\mu}}= & -2 q^{45 / 4}\left(1+q^{3}+q^{6}+q^{10}+q^{14}+q^{18}+q^{23}+2 q^{28}+q^{31}+q^{33}+q^{34}+q^{36}+2 q^{39}+q^{42}\right. \\
& +q^{45}+q^{46}+q^{49}+q^{51}+q^{53}+q^{55}+q^{58}+q^{59}+q^{62}+q^{65}+q^{67}+q^{70}+2 q^{72}+q^{75}+q^{77} \\
& \left.+q^{78}+q^{80}+q^{81}+q^{82}+q^{83}+2 q^{85}+2 q^{88}\right) \ldots
\end{aligned}
$$

For $\mu_{1}=(0,1), \mu_{2}=(1,2)$, we have:

$$
\begin{aligned}
\Phi_{\boldsymbol{\mu}}= & -2 q^{47 / 4}\left(1+q^{3}+q^{5}+q^{9}+q^{13}+q^{17}+q^{23}+q^{28}+q^{29}+q^{31}+q^{32}+q^{35}+q^{36}+q^{37}+q^{38}\right. \\
& +q^{42}+2 q^{44}+q^{49}+q^{50}+q^{52}+q^{53}+2 q^{58}+q^{60}+q^{65}+q^{68}+q^{70}+q^{71}+q^{74}+q^{76} \\
& \left.+q^{77}+q^{78}+q^{79}+q^{80}+q^{83}+q^{84}+3 q^{85}+q^{87}\right)+\ldots
\end{aligned}
$$

For $\mu_{1}=(0,1), \mu_{2}=(1,3)$, we have:

$$
\begin{aligned}
\Phi_{\boldsymbol{\mu}}= & -2 q^{13 / 4}\left(1+q^{6}+q^{10}+q^{15}+q^{19}+q^{20}+q^{21}+q^{27}+q^{30}+q^{33}+q^{34}+q^{35}+q^{39}+q^{42}+q^{45}\right. \\
& +q^{46}+q^{50}+q^{51}+q^{52}+q^{54}+q^{56}+q^{58}+q^{60}+q^{63}+q^{64}+q^{65}+q^{70}+q^{71}+q^{72}+q^{74} \\
& \left.+q^{75}+q^{76}+q^{78}+q^{80}+q^{81}+q^{84}+q^{85}+q^{86}+q^{87}+q^{93}+q^{94}+q^{95}+q^{96}\right)+\ldots
\end{aligned}
$$

For $\mu_{1}=(0,1), \mu_{2}=(2,2)$, we have:

$$
\begin{aligned}
\Phi_{\boldsymbol{\mu}}= & -2 q^{15 / 8}\left(1+q^{10}+q^{13}+q^{15}+q^{16}+2 q^{18}+q^{27}+q^{33}+q^{38}+2 q^{40}+q^{42}+q^{45}+3 q^{47}\right. \\
& +q^{48}+2 q^{52}+2 q^{53}+2 q^{58}+q^{60}+q^{68}+2 q^{69}+q^{75}+2 q^{79}+q^{81}+3 q^{86}+2 q^{88}+q^{90} \\
& \left.+q^{93}+q^{94}+q^{95}+3 q^{96}+2 q^{97}\right)+\ldots
\end{aligned}
$$

For $\mu_{1}=(0,2), \mu_{2}=(0,1)$, we have:

$$
\begin{aligned}
\Phi_{\boldsymbol{\mu}}= & 2 q^{11 / 2}\left(1+q^{4}+q^{10}+2 q^{18}+q^{22}+q^{24}+2 q^{26}+q^{30}+q^{34}+2 q^{36}+q^{42}+q^{44}\right. \\
& +2 q^{48}+2 q^{56}+2 q^{58}+3 q^{60}+q^{64}+2 q^{68}+q^{70}+3 q^{72}+2 q^{74}+q^{80}+3 q^{84}+2 q^{86} \\
& \left.+3 q^{90}+q^{94}\right)+\ldots
\end{aligned}
$$

For $\mu_{1}=(0,2), \mu_{2}=(1,0)$, we have:

$$
\begin{aligned}
\Phi_{\boldsymbol{\mu}}= & 2 q^{41 / 8}\left(1+2 q^{5}+q^{10}+2 q^{11}+4 q^{18}+q^{21}+q^{25}+2 q^{26}+2 q^{27}+2 q^{32}+2 q^{35}+2 q^{36}\right. \\
& +2 q^{37}+q^{41}+2 q^{44}+3 q^{45}+4 q^{48}+2 q^{55}+2 q^{56}+2 q^{57}+q^{58}+2 q^{59}+2 q^{60}+2 q^{61} \\
& +q^{66}+2 q^{68}+3 q^{70}+2 q^{71}+2 q^{73}+2 q^{74}+4 q^{75}+2 q^{81}+2 q^{83}+4 q^{86}+4 q^{87}+q^{88} \\
& \left.+4 q^{90}+2 q^{93}\right)+\ldots
\end{aligned}
$$


For $\mu_{1}=(0,2), \mu_{2}=(1,1)$, we have:

$$
\begin{aligned}
\Phi_{\boldsymbol{\mu}}= & q^{29 / 8}\left(1+q^{4}+3 q^{9}+q^{15}+2 q^{16}+q^{19}+q^{22}+2 q^{23}+2 q^{24}+q^{29}+q^{30}+4 q^{33}+2 q^{38}\right. \\
& +q^{39}+q^{40}+2 q^{42}+2 q^{43}+2 q^{44}+q^{49}+2 q^{51}+q^{52}+3 q^{54}+2 q^{55}+2 q^{56}+q^{60}+2 q^{62} \\
& +3 q^{65}+4 q^{66}+4 q^{69}+q^{70}+q^{72}+2 q^{78}+3 q^{79}+2 q^{80}+2 q^{82}+4 q^{83}+2 q^{84}+q^{85}+q^{87} \\
& \left.+2 q^{91}+2 q^{93}+q^{94}+4 q^{95}+2 q^{96}\right)+\ldots
\end{aligned}
$$

For $\mu_{1}=(0,2), \mu_{2}=(2,0)$, we have:

$$
\begin{aligned}
\Phi_{\boldsymbol{\mu}}= & 4\left(q^{13}+q^{19}+q^{27}+q^{37}+q^{43}+q^{45}+q^{47}+q^{53}+q^{57}+q^{59}+q^{67}+q^{69}+q^{73}+q^{83}\right. \\
& \left.+q^{85}+q^{87}+q^{89}+q^{93}+q^{97}+q^{99}\right)+\ldots
\end{aligned}
$$

List of $\Psi_{\boldsymbol{\mu}}$. Similarly as the $\Phi_{\boldsymbol{\mu}} q$-series we list the $q$-series of $\Psi_{\boldsymbol{\mu}}$ for a various choices of $\boldsymbol{\mu}$. In this example $(-1)^{a+b+c}=(-1)^{\mu_{12}}$. For $\mu_{1}=\mu_{2}=(0,0)$, we have:

$$
\Psi_{\boldsymbol{\mu}}=16\left(q^{6}+4 q^{24}+3 q^{30}+9 q^{54}+12 q^{64}+3 q^{70}+3 q^{78}+16 q^{96}\right)+\ldots
$$

For $\mu_{1}=(0,0) \mu_{2}=(1,0)$, we have:

$$
\begin{aligned}
\Psi_{\boldsymbol{\mu}}= & 2 q^{33 / 8}\left(1+4 q^{4}+9 q^{16}+2 q^{21}+4 q^{22}+16 q^{24}+4 q^{30}+8 q^{31}+q^{42}+25 q^{44}+18 q^{53}+16 q^{54}\right. \\
& \left.+36 q^{56}+2 q^{58}+4 q^{60}+17 q^{66}+40 q^{67}+4 q^{72}+8 q^{74}+8 q^{81}+9 q^{82}+49 q^{84}+2 q^{93}\right)+\ldots
\end{aligned}
$$

For $\mu_{1}=(0,0) \mu_{2}=(2,0)$, we have:

$$
\begin{aligned}
\Psi_{\mu}= & 8 q^{21 / 2}\left(1+q^{6}+q^{12}+4 q^{22}+q^{28}+2 q^{30}+4 q^{32}+2 q^{40}+5 q^{42}+q^{48}+2 q^{54}+9 q^{56}+\right. \\
& \left.+4 q^{66}+8 q^{68}+9 q^{70}+q^{72}+2 q^{76}+10 q^{82}+13 q^{84}\right)+\ldots
\end{aligned}
$$

For $\mu_{1}=(0,0) \mu_{2}=(3,0)$, we have:

$$
\begin{aligned}
\Psi_{\mu}= & 2 q^{105 / 8}\left(5+4 q^{6}+q^{14}+25 q^{24}+4 q^{30}+2 q^{31}+8 q^{33}+16 q^{34}+4 q^{40}+8 q^{45}+9 q^{46}+2 q^{55}\right. \\
& \left.+61 q^{60}+16 q^{70}+18 q^{71}+32 q^{73}+36 q^{74}+4 q^{76}+2 q^{78}+8 q^{82}+16 q^{84}+q^{86}\right)+\ldots
\end{aligned}
$$

For $\mu_{1}=(0,0) \mu_{2}=(0,1)$, we have:

$$
\begin{aligned}
\Psi_{\mu}= & 2 q^{4}\left(1+q^{4}+q^{8}+9 q^{16}+q^{20}+2 q^{22}+9 q^{24}+2 q^{30}+10 q^{32}+q^{36}+2 q^{42}+25 q^{44}\right. \\
& +9 q^{52}+18 q^{54}+26 q^{56}+2 q^{60}+20 q^{66}+34 q^{68}+2 q^{72}+9 q^{76}+2 q 78+2 q^{80}+18 q^{82} \\
& \left.+49 q^{84}+2 q^{92}+2 q^{94}+25 q^{96}\right)+\ldots
\end{aligned}
$$

For $\mu_{1}=(0,0) \mu_{2}=(0,2)$, we have:

$$
\Psi_{\boldsymbol{\mu}}=16\left(q^{16}+q^{32}+4 q^{42}+q^{48}+2 q^{52}+4 q^{66}+2 q^{76}+9 q^{80}+4 q^{90}+8 q^{94}+q^{96}\right)+\ldots
$$

For $\mu_{1}=(0,0) \mu_{2}=(1,1)$, we have:

$$
\begin{aligned}
\Psi_{\boldsymbol{\mu}}= & 2 q^{45 / 8}\left(1+q^{4}+13 q^{18}+q^{22}+2 q^{25}+9 q^{26}+q^{30}+2 q^{32}+4 q^{36}+41 q^{48}+9 q^{56}+8 q^{57}\right. \\
& \left.+18 q^{59}+26 q^{60}+2 q^{66}+9 q^{68}+2 q^{71}+q^{72}+18 q^{73}+16 q^{74}+2 q^{82}+10 q^{85}+85 q^{90}\right)+\ldots
\end{aligned}
$$


For $\mu_{1}=(0,0) \mu_{2}=(1,3)$, we have:

$$
\begin{aligned}
\Psi_{\boldsymbol{\mu}}= & 2 q^{21 / 8}\left(1+4 q^{8}+q^{12}+9 q^{14}+q^{18}+2 q^{19}+16 q^{30}+8 q^{37}+9 q^{38}+25 q^{40}+q^{42}+9 q^{48}\right. \\
& +20 q^{49}+q^{52}+2 q^{54}+2 q^{61}+4 q^{62}+36 q^{64}+32 q^{75}+25 q^{76}+49 q^{78}+8 q^{82}+9 q^{84}+q^{88} \\
& \left.+29 q^{90}+68 q^{91}\right) \ldots
\end{aligned}
$$

For $\mu_{1}=(0,0) \mu_{2}=(2,1)$, we have:

$$
\begin{aligned}
\Psi_{\boldsymbol{\mu}}= & 4 q^{15 / 2}\left(1+q^{12}+9 q^{20}+q^{24}+2 q^{28}+9 q^{40}+2 q^{48}+25 q^{52}+9 q^{60}+19 q^{64}+2 q^{72}+2 q^{76}\right. \\
& \left.+25 q^{80}+18 q^{92}\right)+\ldots
\end{aligned}
$$

For $\mu_{1}=(0,0) \mu_{2}=(2,3)$, we have:

$$
\Psi_{\boldsymbol{\mu}}=4 q^{3 / 2}\left(1+9 q^{12}+3 q^{16}+25 q^{36}+27 q^{44}+3 q^{48}+3 q^{56}+49 q^{72}+75 q^{84}+27 q^{92}+3 q^{96}\right)+\ldots
$$

For $\mu_{1}=(0,2) \mu_{2}=(2,0)$, we have:

$$
\begin{aligned}
\Psi_{\boldsymbol{\mu}}= & 4\left(q^{13}+q^{19}+q^{27}+9 q^{37}+q^{43}+q^{45}+9 q^{47}+q^{53}+q^{57}+9 q^{59}+q^{67}+q^{69}\right. \\
& \left.+25 q^{73}+9 q^{83}+9 q^{85}+25 q^{87}+q^{89}+q^{93}+9 q^{97}+q^{99}\right)+\ldots
\end{aligned}
$$

For $\mu_{1}=(0,1) \mu_{2}=(1,0)$, we have:

$$
\begin{aligned}
\Psi_{\boldsymbol{\mu}}= & -\frac{q^{19 / 4}}{2}\left(1+q^{2}+q^{6}+25 q^{17}+26 q^{21}+q^{23}+9 q^{24}+q^{26}+q^{27}+25 q^{29}+q^{33}+9 q^{34}+q^{38}\right. \\
& +9 q^{39}+81 q^{46}+81 q^{52}+25 q^{54}+25 q^{56}+49 q^{57}+q^{58}+25 q^{61}+26 q^{62}+90 q^{64}+q^{66}+2 q^{68} \\
& +49 q^{71}+25 q^{72}+q^{75}+q^{76}+25 q^{77}+49 q^{78}+9 q^{81}+9 q^{82}+q^{86}+169 q^{87}+9 q^{88}+q^{89} \\
& \left.+9 q^{90}+169 q^{95}\right)+\ldots
\end{aligned}
$$

For $\mu_{1}=(0,1) \mu_{2}=(2,0)$, we have:

$$
\begin{aligned}
\Psi_{\boldsymbol{\mu}}= & -\frac{q^{71 / 8}}{2}\left(1+q^{5}+9 q^{9}+q^{12}+9 q^{16}+25 q^{21}+9 q^{25}+q^{26}+q^{28}+25 q^{30}+q^{35}+49 q^{36}\right. \\
& +q^{39}+25 q^{41}+9 q^{43}+9 q^{45}+49 q^{47}+q^{48}+q^{50}+90 q^{54}+9 q^{58}+49 q^{60}+25 q^{63} \\
& +25 q^{65}+81 q^{67}+q^{68}+9 q^{69}+9 q^{71}+q^{72}+121 q^{75}+25 q^{76}+q^{78}+25 q^{80} \\
& \left.+q^{81}+81 q^{82}+49 q^{86}+49 q^{88}+q^{89}+121 q^{90}\right)+\ldots
\end{aligned}
$$

For $\mu_{1}=(0,1) \mu_{2}=(0,0)$, we have:

$$
\begin{aligned}
\Psi_{\boldsymbol{\mu}}= & -\frac{q^{39 / 8}}{2}\left(1+9 q^{2}+q^{3}+25 q^{17}+49 q^{21}+2 q^{22}+25 q^{24}+9 q^{26}+q^{27}+18 q^{28}+2 q^{33}+9 q^{39}\right. \\
& +81 q^{46} 121 q^{52}+50 q^{55}+81 q^{57}+2 q^{60}+49 q^{61}+98 q^{63}+25 q^{64}+9 q^{66}+q^{67}+q^{68} \\
& \left.+68 q^{70}+18 q^{76}+49 q^{78}+2 q^{79}+2 q^{81}+169 q^{87}+18 q^{89}+225 q^{95}\right)+\ldots
\end{aligned}
$$


For $\mu_{1}=(0,1) \mu_{2}=(1,1)$, we have:

$$
\begin{aligned}
\Psi_{\boldsymbol{\mu}}= & -\frac{q^{45 / 4}}{2}\left(1+q^{3}+q^{6}+9 q^{10}+9 q^{14}+9 q^{18}+25 q^{23}+26 q^{28}+q^{31}+25 q^{33}+q^{34}+q^{36}\right. \\
& +50 q^{39}+q^{42}+49 q^{45}+9 q^{46}+9 q^{49}+49 q^{51}+9 q^{53}+9 q^{55}+81 q^{58}+9 q^{59}+9 q^{62}+81 q^{65} \\
& \left.+25 q^{67}+25 q^{70}+82 q^{72}+25 q^{75}+25 q^{77}+q^{78}+121 q^{80}+q^{81}+25 q^{82}+q^{83}+26 q^{85}+122 q^{88}\right) \\
& +\ldots
\end{aligned}
$$

For $\mu_{1}=(0,1) \mu_{2}=(1,2)$, we have:

$$
\begin{aligned}
\Psi_{\boldsymbol{\mu}}= & -\frac{q^{47 / 4}}{2}\left(9+9 q^{3}+q^{5}+9 q^{9}+q^{13}+q^{17}+49 q^{23}+49 q^{28}+9 q^{29}+9 q^{31}+25 q^{32}+9 q^{35}\right. \\
& +9 q^{36}+q^{37}+49 q^{38}+q^{42}+34 q^{44}+9 q^{49}+25 q^{50}+q^{52}+q^{53}+122 q^{58}+q^{60}+121 q^{65}+49 q^{68} \\
& \left.+49 q^{70}+81 q^{71}+9 q^{74}+49 q^{76}+49 q^{77}+9 q^{78}+121 q^{79}+25 q^{80}+9 q^{83}+9 q^{84}+25 q^{85}+81 q^{87}\right) \\
& +\ldots
\end{aligned}
$$

For $\mu_{1}=(0,1) \mu_{2}=(1,3)$, we have:

$$
\begin{aligned}
\Psi_{\boldsymbol{\mu}}= & -\frac{q^{13 / 4}}{2}\left(1+9 q^{6}+q^{10}+25 q^{15}+q^{19}+q^{20}+9 q^{21}+49 q^{27}+q^{30}+9 q^{33}+9 q^{34}+25 q^{35}\right. \\
& +q^{39}+81 q^{42}+q^{45}+9 q^{46}+25 q^{50}+25 q^{51}+49 q^{52}+q^{54}+q^{56}+9 q^{58}+121 q^{60} \\
& +q^{63}+9 q^{64}+25 q^{65}+49 q^{70}+49 q^{71}+81 q^{72}+q^{74}+q^{75}+9 q^{76}+9 q^{78}+25 q^{80} \\
& \left.+169 q^{81}+q^{84}+9 q^{85}+25 q^{86}+49 q^{87}+81 q^{93}+81 q^{94}+121 q^{95}+q^{96}\right)+\ldots
\end{aligned}
$$

For $\mu_{1}=(0,1) \mu_{2}=(2,2)$, we have:

$$
\begin{aligned}
\Psi_{\boldsymbol{\mu}}= & -\frac{q^{15 / 8}}{2}\left(1+9 q^{10}+25 q^{13}+9 q^{15}+q^{16}+2 q^{18}+q^{27}+49 q^{33}+81 q^{38}+18 q^{40}+49 q^{42}+25 q^{45}\right. \\
& +59 q^{47}+q^{48}+2 q^{52}+18 q^{53}+2 q^{58}+25 q^{60}+121 q^{68}+2 q^{69}+169 q^{75}+98 q^{79}+121 q^{81} \\
& \left.+99 q^{86}+162 q^{88}+49 q^{90}+25 q^{93}+9 q^{94}+9 q^{95}+99 q^{96}+50 q^{97}\right)+\ldots
\end{aligned}
$$

For $\mu_{1}=(0,2) \mu_{2}=(0,1)$, we have:

$$
\Psi_{\boldsymbol{\mu}}=16 q^{47 / 2}\left(1+q^{8}+q^{18}+4 q^{30}+q^{38}+q^{40}+4 q^{42}+q^{50}+q^{54}+4 q^{56}+q^{66}+q^{68}+9 q^{72}\right)+\ldots
$$

For $\mu_{1}=(0,2) \mu_{2}=(1,0)$, we have:

$$
\begin{aligned}
\Psi_{\mu}= & 2 q^{81 / 8}\left(1+q^{6}+5 q^{13}+q^{21}+9 q^{22}+q^{27}+q^{30}+4 q^{31}+9 q^{32}+q^{39}+q^{40}+25 q^{43}+4 q^{50}\right. \\
& +q^{51}+q^{52}+4 q^{54}+9 q^{55}+25 q^{56}+q^{63}+9 q^{65}+q^{66}+9 q^{68}+16 q^{69}+26 q^{70}+q^{76} \\
& \left.+4 q^{78}+10 q^{81}+13 q^{82}+61 q^{85}+q^{88}\right)+\ldots
\end{aligned}
$$

For $\mu_{1}=(0,2) \mu_{2}=(1,1)$, we have:

$$
\begin{aligned}
\Psi_{\mu}= & 2 q^{101 / 8}\left(1+4 q^{7}+q^{14}+4 q^{15}+13 q^{24}+q^{29}+q^{33}+4 q^{34}+16 q^{35}+4 q^{42}+4 q^{45}+9 q^{46}\right. \\
& +16 q^{47}+q^{53}+4 q^{56}+5 q^{57}+41 q^{60}+9 q^{69}+4 q^{70}+4 q^{71}+9 q^{73}+17 q^{74} \\
& \left.+36 q^{75}+q^{82}+4 q^{84}+17 q^{86}+4 q^{87}\right)+\ldots
\end{aligned}
$$




\section{Acknowledgments}

We thank Sergey Alexandrov and Boris Pioline for useful discussions and comments on the draft. The authors are supported by Laureate Award 15175 "Modularity in Quantum Field Theory and Gravity" of the Irish Research Council.

Open Access. This article is distributed under the terms of the Creative Commons Attribution License (CC-BY 4.0), which permits any use, distribution and reproduction in any medium, provided the original author(s) and source are credited.

\section{References}

[1] J.M. Maldacena, J. Michelson and A. Strominger, Anti-de Sitter fragmentation, JHEP 02 (1999) 011 [hep-th/9812073] [INSPIRE].

[2] A. Maloney, M. Spradlin and A. Strominger, Superconformal multiblack hole moduli spaces in four-dimensions, JHEP 04 (2002) 003 [hep-th/9911001] [INSPIRE].

[3] F. Denef, Supergravity flows and D-brane stability, JHEP 08 (2000) 050 [hep-th/0005049] [INSPIRE].

[4] F. Denef, B.R. Greene and M. Raugas, Split attractor flows and the spectrum of BPS D-branes on the quintic, JHEP 05 (2001) 012 [hep-th/0101135] [INSPIRE].

[5] I. Bena, C.-W. Wang and N.P. Warner, Mergers and typical black hole microstates, JHEP 11 (2006) 042 [hep-th/0608217] [INSPIRE].

[6] F. Denef and G.W. Moore, Split states, entropy enigmas, holes and halos, JHEP 11 (2011) 129 [hep-th/0702146] [INSPIRE].

[7] M.C.N. Cheng and E. Verlinde, Dying Dyons Don't Count, JHEP 09 (2007) 070 [arXiv: 0706.2363] [INSPIRE].

[8] J. de Boer, S. El-Showk, I. Messamah and D. Van den Bleeken, Quantizing $N=2$ Multicenter Solutions, JHEP 05 (2009) 002 [arXiv:0807.4556] [inSPIRE].

[9] J. Manschot, B. Pioline and A. Sen, Wall Crossing from Boltzmann Black Hole Halos, JHEP 07 (2011) 059 [arXiv: 1011.1258] [INSPIRE].

[10] A. Dabholkar, S. Murthy and D. Zagier, Quantum Black Holes, Wall Crossing, and Mock Modular Forms, arXiv: 1208.4074 [INSPIRE].

[11] I. Bena, M. Berkooz, J. de Boer, S. El-Showk and D. Van den Bleeken, Scaling BPS Solutions and pure-Higgs States, JHEP 11 (2012) 171 [arXiv:1205.5023] [INSPIRE].

[12] J.M. Maldacena, A. Strominger and E. Witten, Black hole entropy in M-theory, JHEP 12 (1997) 002 [hep-th/9711053] [INSPIRE].

[13] D. Gaiotto, A. Strominger and X. Yin, The M5-Brane Elliptic Genus: Modularity and BPS States, JHEP 08 (2007) 070 [hep-th/0607010] [INSPIRE].

[14] J. de Boer, M.C.N. Cheng, R. Dijkgraaf, J. Manschot and E. Verlinde, A Farey Tail for Attractor Black Holes, JHEP 11 (2006) 024 [hep-th/0608059] [INSPIRE].

[15] P. Kraus and F. Larsen, Partition functions and elliptic genera from supergravity, JHEP 01 (2007) 002 [hep-th/0607138] [INSPIRE].

[16] S. Alexandrov, J. Manschot and B. Pioline, D3-instantons, Mock Theta Series and Twistors, JHEP 04 (2013) 002 [arXiv: 1207.1109] [InSPIRE]. 
[17] S. Alexandrov, J. Manschot, D. Persson and B. Pioline, Quantum hypermultiplet moduli spaces in $N=2$ string vacua: a review, Proc. Symp. Pure Math. 90 (2015) 181 [arXiv:1304.0766] [INSPIRE].

[18] S. Alexandrov, S. Banerjee, J. Manschot and B. Pioline, Multiple D3-instantons and mock modular forms I, Commun. Math. Phys. 353 (2017) 379 [arXiv: 1605. 05945] [InSPIRE].

[19] A. Gholampour and A. Sheshmani, Donaldson-Thomas invariants of 2-dimensional sheaves inside threefolds and modular forms, Adv. Math. 326 (2018) 79 [arXiv:1309.0050] [INSPIRE].

[20] A. Gholampour, A. Sheshmani and R. Thomas, Counting curves on surfaces in Calabi-Yau 3-folds, Math. Ann. 360 (2014) 67 [arXiv:1309.0051] [InSPIRE].

[21] V. Bouchard, T. Creutzig, D.-E. Diaconescu, C. Doran, C. Quigley and A. Sheshmani, Vertical D4-D2-D0 Bound States on K3 Fibrations and Modularity, Commun. Math. Phys. 350 (2017) 1069 [arXiv: 1601.04030] [INSPIRE].

[22] S. Feyzbakhsh and R.P. Thomas, Curve counting and S-duality, arXiv:2007.03037 [INSPIRE].

[23] S. Feyzbakhsh and R.P. Thomas, Rank $r$ DT theory from rank 0, arXiv:2103.02915 [INSPIRE].

[24] E. Andriyash and G.W. Moore, Ample D4-D2-D0 Decay, arXiv:0806.4960 [InSPIRE].

[25] J. Manschot, Stability and duality in $N=2$ supergravity, Commun. Math. Phys. 299 (2010) 651 [arXiv:0906.1767] [INSPIRE].

[26] J. Manschot, Wall-crossing of D4-branes using flow trees, Adv. Theor. Math. Phys. 15 (2011) 1 [arXiv: 1003.1570] [INSPIRE].

[27] J. de Boer, F. Denef, S. El-Showk, I. Messamah and D. Van den Bleeken, Black hole bound states in $A d S_{3} \times S^{2}$, JHEP 11 (2008) 050 [arXiv:0802.2257] [INSPIRE].

[28] S. Alexandrov, S. Banerjee, J. Manschot and B. Pioline, Multiple D3-instantons and mock modular forms II, Commun. Math. Phys. 359 (2018) 297 [arXiv:1702.05497] [INSPIRE].

[29] S. Alexandrov and B. Pioline, Black holes and higher depth mock modular forms, Commun. Math. Phys. 374 (2019) 549 [arXiv:1808.08479] [inSPIRE].

[30] S. Alexandrov, J. Manschot and B. Pioline, S-duality and refined BPS indices, Commun. Math. Phys. 380 (2020) 755 [arXiv: 1910.03098] [INSPIRE].

[31] D. Robles-Llana, M. Roček, F. Saueressig, U. Theis and S. Vandoren, Nonperturbative corrections to $4 D$ string theory effective actions from $\mathrm{SL}(2, Z)$ duality and supersymmetry, Phys. Rev. Lett. 98 (2007) 211602 [hep-th/0612027] [INSPIRE].

[32] S. Alexandrov, B. Pioline, F. Saueressig and S. Vandoren, D-instantons and twistors, JHEP 03 (2009) 044 [arXiv: 0812 .4219] [INSPIRE].

[33] S. Alexandrov, D-instantons and twistors: Some exact results, J. Phys. A 42 (2009) 335402 [arXiv:0902.2761] [INSPIRE].

[34] S. Zwegers, Mock Theta Functions, Ph.D. Thesis, Utrecht University (2008). [arXiv: 0807.4834] [INSPIRE].

[35] D. Zagier, Ramanujan's mock theta functions and their applications (after Zwegers and Ono-Bringmann), Astérisque 326 (2009) Exp. No. 986.

[36] S. Alexandrov, S. Banerjee, J. Manschot and B. Pioline, Indefinite theta series and generalized error functions, Selecta Math. 24 (2018) 3927 [arXiv:1606. 05495] [INSPIRE].

[37] C. Nazaroglu, r-Tuple Error Functions and Indefinite Theta Series of Higher-Depth, Commun. Num. Theor. Phys. 12 (2018) 581 [arXiv:1609.01224] [INSPIRE]. 
[38] S. Kudla, Theta integrals and generalized error functions, Manuscripta Math. 155 (2016) 303 [arXiv: 1608.03534].

[39] J. Funke and S. Kudla, On some incomplete theta integrals, Compos. Math. 155 (2019) 1711.

[40] C. Vafa and E. Witten, A Strong coupling test of S duality, Nucl. Phys. B 431 (1994) 3 [hep-th/9408074] [INSPIRE].

[41] J.A. Minahan, D. Nemeschansky, C. Vafa and N.P. Warner, E strings and $N=4$ topological Yang-Mills theories, Nucl. Phys. B 527 (1998) 581 [hep-th/9802168] [InSPIRE].

[42] J. Manschot, BPS invariants of $\mathcal{N}=4$ gauge theory on Hirzebruch surfaces, Commun. Num. Theor. Phys. 6 (2012) 497 [arXiv:1103.0012] [inSPIRE].

[43] J. Manschot, Vafa-Witten Theory and Iterated Integrals of Modular Forms, Commun. Math. Phys. 371 (2019) 787 [arXiv: 1709.10098] [inSPIRE].

[44] A. Dabholkar, P. Putrov and E. Witten, Duality and Mock Modularity, SciPost Phys. 9 (2020) 072 [arXiv: 2004.14387] [INSPIRE].

[45] S. Alexandrov, Vafa-Witten invariants from modular anomaly, Commun. Num. Theor. Phys. 15 (2021) 149 [arXiv: 2005.03680] [INSPIRE].

[46] S. Alexandrov, Rank N Vafa-Witten invariants, modularity and blow-up, arXiv:2006.10074 [INSPIRE].

[47] G. Bonelli, F. Fucito, J.F. Morales, M. Ronzani, E. Sysoeva and A. Tanzini, Gauge theories on compact toric manifolds, Lett. Math. Phys. 111 (2021) 77 [arXiv:2007.15468] [INSPIRE].

[48] J. Manschot and G.W. Moore, Topological correlators of $\mathrm{SU}(2), \mathcal{N}=2^{*} S Y M$ on four-manifolds, arXiv:2104.06492 [INSPIRE].

[49] S.-J. Lee, Z.-L. Wang and P. Yi, Quiver Invariants from Intrinsic Higgs States, JHEP 07 (2012) 169 [arXiv: 1205.6511] [INSPIRE].

[50] D. Anninos, T. Anous, P. de Lange and G. Konstantinidis, Conformal quivers and melting molecules, JHEP 03 (2015) 066 [arXiv:1310.7929] [INSPIRE].

[51] J. Manschot, B. Pioline and A. Sen, On the Coulomb and Higgs branch formulae for multi-centered black holes and quiver invariants, JHEP 05 (2013) 166 [arXiv:1302.5498] [INSPIRE].

[52] D. Mirfendereski, J. Raeymaekers and D. Van Den Bleeken, Superconformal mechanics of $A d S_{2}$ D-brane boundstates, JHEP 12 (2020) 176 [arXiv: 2009.07107] [INSPIRE].

[53] G. Beaujard, S. Mondal and B. Pioline, Multi-centered black holes, scaling solutions and pure-Higgs indices from localization, SciPost Phys. 11 (2021) 023 [arXiv:2103.03205] [INSPIRE].

[54] J. de Boer, J. Manschot, K. Papadodimas and E. Verlinde, The Chiral ring of $A d S_{3} / C F T_{2}$ and the attractor mechanism, JHEP 03 (2009) 030 [arXiv: 0809.0507] [INSPIRE].

[55] M.-F. Vignéras, Séries thêta des formes quadratiques indéfinies, in Modular Functions of One Variable VI, Lecture Notes in Mathematics, vol. 627, Springer, Berlin, Heidelberg (1977) [DOI].

[56] J. Manschot and G.W. Moore, A Modern Farey Tail, Commun. Num. Theor. Phys. 4 (2010) 103 [arXiv: 0712.0573] [INSPIRE].

[57] K. Bringmann, J. Kaszian and A. Milas, Higher depth quantum modular forms, multiple eichler integrals, and $\mathfrak{s l}_{3}$ false theta functions, Res. Math. Sci. 6 (2019) 20 [arXiv:1704.06891].

[58] B. Pioline, Wall-crossing made smooth, JHEP 04 (2015) 092 [arXiv:1501.01643] [inSPIRE]. 
[59] S.S. Kudla and J.J. Millson, The theta correspondence and harmonic forms. I, Math. Ann. 274 (1986) 353.

[60] N. Afkhami-Jeddi, H. Cohn, T. Hartman and A. Tajdini, Free partition functions and an averaged holographic duality, JHEP 01 (2021) 130 [arXiv: 2006.04839] [INSPIRE].

[61] A. Maloney and E. Witten, Averaging over Narain moduli space, JHEP 10 (2020) 187 [arXiv: 2006.04855] [INSPIRE].

[62] S. Ferrara, R. Kallosh and A. Strominger, $N=2$ extremal black holes, Phys. Rev. D 52 (1995) R5412 [hep-th/9508072] [INSPIRE].

[63] S. Ferrara and R. Kallosh, Supersymmetry and attractors, Phys. Rev. D 54 (1996) 1514 [hep-th/9602136] [INSPIRE].

[64] M. Shmakova, Calabi-Yau black holes, Phys. Rev. D 56 (1997) 540 [hep-th/9612076] [INSPIRE].

[65] F. Denef, Quantum quivers and Hall/hole halos, JHEP 10 (2002) 023 [hep-th/0206072] [INSPIRE].

[66] B. Bates and F. Denef, Exact solutions for supersymmetric stationary black hole composites, JHEP 11 (2011) 127 [hep-th/0304094] [InSPIRE]

[67] J. Manschot, B. Pioline and A. Sen, The Coulomb Branch Formula for Quiver Moduli Spaces, arXiv: 1404.7154 [INSPIRE].

[68] J. Manschot, B. Pioline and A. Sen, A Fixed point formula for the index of multi-centered $N=2$ black holes, JHEP 05 (2011) 057 [arXiv:1103.1887] [INSPIRE].

[69] S.-J. Lee and P. Yi, Witten Index for Noncompact Dynamics, JHEP 06 (2016) 089 [arXiv: 1602.03530] [INSPIRE].

[70] S. Alexandrov and B. Pioline, Attractor flow trees, BPS indices and quivers, Adv. Theor. Math. Phys. 23 (2019) 627 [arXiv: 1804.06928] [INSPIRE].

[71] H. Ooguri, A. Strominger and C. Vafa, Black hole attractors and the topological string, Phys. Rev. D 70 (2004) 106007 [hep-th/0405146] [INSPIRE].

[72] J. Conway and N. Sloane, Sphere packings, lattices and groups, Springer (1999) [DOI].

[73] T. Gannon, Lattices and Superstrings, Ph.D. Thesis, McGill University (1989).

[74] T.-T. Lu and S.-H. Shiou, Inverses of $2 \times 2$ Block Matrices, Comput. Math. Appl. 43 (2002) 119.

[75] J. Funke and S. Kudla, The case of an N-gon, arXiv:2109.10979.

[76] R. Minasian, G.W. Moore and D. Tsimpis, Calabi-Yau black holes and $(0,4)$ sigma models, Commun. Math. Phys. 209 (2000) 325 [hep-th/9904217] [inSPIRE].

[77] Toric calabi-yau database, http://rossealtman.com/toriccy/.

[78] D. Maulik and R. Pandharipande, Gromov-Witten theory and Noether-Lefschetz theory, arXiv:0705.1653

[79] D. Gaiotto and X. Yin, Examples of M5-Brane Elliptic Genera, JHEP 11 (2007) 004 [hep-th/0702012] [INSPIRE].

[80] A. Klemm, M. Kreuzer, E. Riegler and E. Scheidegger, Topological string amplitudes, complete intersection Calabi-Yau spaces and threshold corrections, JHEP 05 (2005) 023 [hep-th/0410018] [INSPIRE]. 